\title{
Uniformizing complex ODEs and applications
}

\author{
Julio C. Rebelo and Helena Reis
}

\begin{abstract}
We introduce a method for estimating the size of the domain of definition of the solutions of a meromorphic vector field on a neighborhood of its pole divisor. The technique relies, in a certain sense, on obtaining a quantitative variant of some well-known results concerning the distance function between complex submanifolds in the presence of metrics with positive curvature. Several applications of these ideas are provided including a type of "confinement theorem" for the solutions of the differential equations associated to complete polynomial vector fields on $\mathbb{C}^{n}$ as well as obstructions to realizing certain germs of vector fields as a singularity of a globally defined holomorphic vector field on a compact Kähler manifold. As a complement, a new approach to certain classical equations is proposed and detailed in the case of Halphen equations.
\end{abstract}

\section{Introduction}

The object of this paper is a method to investigate the domain of definition of the solutions of a differential equation associated with a meromorphic vector field (to abridge notation, we shall refer to these functions as solutions of meromorphic vector fields). The method is quite general in that it applies to arbitrarily high dimensions whereas it provides new results already in dimension 3. This paper has two parts, the first presents the general setup along with basic estimates and results. Applications of this material are then provided in the second part.

This introduction is aimed only at stating the main applications considered in this paper. These were chosen to indicate ways of exploiting the basic phenomena on which our analysis relies and are not intended to be the sharpest possible. In Section 2, we shall provide a more detailed discussion explaining our point of view and underlining the common structures behind the theorems stated below. It is also to be noted that these applications concern very special types of vector fields (or of differential equations) such as complete vector fields and Halphen equations. Nonetheless the setting is also well adapted to investigating differential equations having meromorphic solutions including several classical equations appearing in

Mathematics Subject Classification (2010): 37F75, 34M05, 34M55.

Keywords: Complex ODE, maximal domain of solutions, entire solutions, Halphen equations. 
mathematics and physics, for example equations arising in the works of Painlevé and Chazy as well as certain Lorenz systems [38]. Through the work of Ablowitz, Segur and others, some of our statements can also be adapted to solutions of certain nonlinear evolution equations and to solutions of linear integral equations in Gelfand-Levitan-Marcenko theory, see [1], [2]. More details on these issues can be found in Section 2. Whereas these connections will not be developed here, they provide a clear indication that "further applications" are likely to be found in the future.

Let $X$ be a polynomial vector field on $\mathbb{C}^{n}$ of degree at least 2 . Suppose that $X$ is complete, i.e., its complex solutions are defined for all $T \in \mathbb{C}$. When $X$ happens to be completely integrable, i.e., when it admits $n-1$ independent first integrals, its orbits can be compactified into rational curves by adding to them some "singular points at infinity". This fact can be interpreted as a type of confinement phenomenon for the corresponding solutions. Our first result is a sharp, whereas weaker, generalization of this confinement phenomenon to arbitrary complete polynomial vector fields. To state it, we proceed as follows. Being polynomial, $X$ defines a singular holomorphic foliation $\mathcal{D}$ on $\mathbb{C P}^{n}=\mathbb{C}^{n} \cup \Delta_{\infty}$ viewed as a compactification of $\mathbb{C}^{n}$. Consider a leaf $L$ of $\mathcal{D}$ (details on the definition of "leaf" in the singular context can be found in Section 2.2). On $L$ two singular oriented real one-dimensional foliations $\mathcal{H}$ and $\mathcal{H}^{\perp}$ will be defined. They will depend on the leaf $L$ of $\mathcal{D}$ in a regular way as will be apparent from their definitions; see Sections 3 and 6 . More importantly, $\mathcal{H}$ and $\mathcal{H}^{\perp}$ are mutually orthogonal with respect to the conformal structure on $L$. In fact, they agree respectively with the real foliation and the purely imaginary foliation induced on $L$ by a certain Abelian form. Since $L$ is endowed with a conformal structure, it also makes sense to define foliations $\mathcal{H}^{\theta}$ whose oriented trajectories make an angle $\theta$ with the oriented trajectories of $\mathcal{H}(\theta \in[-\pi / 2, \pi / 2])$. The trajectories of these foliations define the "directions of confinement" for $L$ as will follow from Theorem A below. In the sequel $\Phi: \mathbb{C} \times \mathbb{C}^{n} \rightarrow \mathbb{C}^{n}$ stands for the holomorphic flow generated by $X$, while Sing $(\mathcal{D}) \subset \mathbb{C P}^{n}$ denotes the singular set of $\mathcal{D}$.

Theorem A. Suppose that $X$ is a complete polynomial vector field on $\mathbb{C}^{n}$ of degree at least 2. Fix an arbitrarily small neighborhood $V$ of $\left(\operatorname{Sing}(\mathcal{D}) \cap \Delta_{\infty}\right) \cup \operatorname{Sing}(X)$ in $\mathbb{C P}^{n}$ and suppose given a point $p \in \mathbb{C}^{n}$ such that $X(p) \neq 0$, and an angle $\theta \in(-\pi / 2, \pi / 2)$. Denote by $L_{p}\left(\right.$ resp. $\left.l_{p}^{+, \theta}\right)$ the leaf of $\mathcal{D}$ through $p$ (resp. the semitrajectory of $\mathcal{H}^{\theta}$ initiated at $\left.p\right)$ and consider the lift $c:[0, \infty) \rightarrow \mathbb{C}$ of $l_{p}^{+, \theta}$ by $\Phi$; i.e., $t \in[0, \infty) \mapsto \Phi(c(t), p)$ is a one-to-one parametrization of $l_{p}^{+, \theta}(c(0)=0)$. Then there is a constant $C$ such that

$$
\text { meas }(\{t \in[0, \infty) ; \Phi(c(t), p) \notin V\})<C,
$$

where meas stands for the usual Lebesgue measure on $\mathbb{R}$.

The preceding theorem states that the trajectory $l_{p}^{+, \theta}$ spend most of its "life" in the neighborhood $V$ and hence arbitrarily close to the singular points of $\mathcal{D}$ or of $X$. Furthermore the constant $C$ varies continuously with $\theta$. In particular, if a 
compact interval $[-\pi / 2+\delta, \pi / 2-\delta] \subset(-\pi / 2, \pi / 2)$ is fixed, then $C$ can be chosen so that the above estimate holds for every $\theta \in[-\pi / 2+\delta, \pi / 2-\delta]$. The uniform dependence of $C$ on $\theta \in[-\pi / 2+\delta, \pi / 2-\delta]$ allows us to generalize the statement to paths $c \subset L_{p}$ more general than the trajectories of $\mathcal{H}^{\theta}$. For example, we may consider paths $c$ as before such that the angle made at the point $c(t)$ by the tangent vector $c^{\prime}(t)$ and the foliation $\mathcal{H}$ lies in $[-\pi / 2+\delta, \pi / 2-\delta]$ for all $t$. The interested reader will have no difficulty in adapting the statement of Theorem $\mathrm{A}$ to these more general situations.

Confinement phenomena are in stark contrast with ergodicity so that it is natural to search for a variant of Theorem A focusing on the "area" of the region defined in $\mathbb{C}$ by those values of $T$ for which $\Phi(T, p) \in V$. This variant might be viewed, in particular, as a "super nonergodic" phenomenon for complete vector fields. To state it, let $B_{r} \subset \mathbb{C}$ denote the disc of radius $r$ centered on $0 \in \mathbb{C}$. A continuous properly embedded path $c:(-\infty, \infty) \rightarrow \mathbb{C}$ is said to be a separating curve if it is of class $C^{\infty}$, except possibly on a discrete set, and if it either is periodic or satisfies the conditions $\lim _{t \rightarrow-\infty} c(t)=\infty$ and $\lim _{t \rightarrow \infty} c(t)=\infty$. Here, the condition $\lim _{t \rightarrow \pm \infty} c(t)=\infty$ simply means that the curve eventually leaves every compact subset of $\mathbb{C}$. A separating curve divides $\mathbb{C}$ in at least two connected components with at least one of these components unbounded. Then we have:

Theorem A'. Let $X, V, L_{p}$ and $p \in \mathbb{C}^{n}$ be as in the statement of Theorem A. Consider the parametrization of $L_{p}$ by $\mathbb{C}$ (possibly as a covering map) which is given by $\Phi_{p}(T)=\Phi(T, p)$. Then there exists a separating curve $c:(-\infty, \infty) \rightarrow \mathbb{C}$, $\Phi_{p}(c(0))=p$, and an unbounded component $\mathcal{U}^{+}$of $\mathbb{C} \backslash c(t)$ such that the following holds: the set $\mathcal{T}_{V} \subset \mathcal{U}^{+} \subset \mathbb{C}$ defined by

$$
\mathcal{T}_{V}=\left\{T \in \mathcal{U}^{+} \subset \mathbb{C} ; \Phi(T, p) \in V\right\}
$$

satisfies

$$
\lim _{r \rightarrow \infty} \frac{\operatorname{Meas}\left(\mathcal{T}_{V} \cap B_{r}\right)}{\operatorname{Meas}\left(\mathcal{U}^{+} \cap B_{r}\right)}=1,
$$

where Meas stands for the usual Lebesgue measure of $\mathbb{C}\left(\simeq \mathbb{R}^{2}\right)$.

The above statement contrasts markedly with various equidistribution results obtained by Fornaess-Sibony and also studied by X. Gómez-Mont and his collaborators; see [18], [17] and [5]. The reader will note that these authors work in a generic setting having "hyperbolic nature" whereas the previous statements are closer to the nongeneric "parabolic" case.

Unlike most standard averaging theorems, the statement above holds for every point $p \in \mathbb{C}^{n}$ and not only for almost every point. Moreover, it is easy to conclude from the proof given in Section 6.1 that for almost all points $p$ the corresponding separating curve is smooth.

This separating curve turns out to have a natural interpretation as a geodesic of a suitable singular flat structure on $\mathbb{C}$. Furthermore, this (singular) flat structure on $\mathbb{C}$ has "bounded geometry" in a natural sense despite the noncompactness of $\mathbb{C}$. The nature of this "bounded geometry" issue deserves some additional 
comments (the reader is referred to Section 6.1 for details). The notion of bounded geometry is, indeed, related to the analogous statement concerning the leaves of a (regular) foliation defined on a compact manifold; see for example [33]. More precisely, consider for a regular foliation defined on some compact manifold $M$ and some geometric object defined on $M$, the simplest example being a Riemannian metric. The restriction of this metric to a noncompact leaf $L$ of the foliation must have "bounded geometry" which, in the case of a Riemannian metric, means that the injectivity radius and all the standard curvatures are bounded. Since $L$ is noncompact, not every Riemannian metric defined on $L$ needs to have "bounded geometry": the point of the preceding observation is that metrics induced on $L$ from the ambient compact manifold $M$ always belong to this distinguished class of metrics. Furthermore, this observation boils down to the fact that the coefficients of the metric are, ultimately, defined on the compact manifold $M$ and therefore are "bounded" in a natural sense. Since only the compactness of the ambient manifold $M$ plays a role in the discussion, the same argument also applies for "foliated objects", such as Riemannian metrics defined only on the tangent bundle of the foliation or, more directly, defined on the corresponding leaves (provided that they vary "continuously" from leaf to leaf). Clearly, none of this needs to hold if the ambient manifold $M$ is not compact. Having recalled these simple facts, let us go back to our complete polynomial vector field defined on $\mathbb{C}^{n}$. Although $\mathbb{C}^{n}$ is not compact, it can be compactified into $\mathbb{C P}^{n}$ and, as already pointed out, the foliation $\mathcal{D}$ associated to $X$ extends to $\mathbb{C P}^{n}$. As far as "bounded geometry" for the leaves of $\mathcal{D}$ is concerned, the issue is then to decide whether or not the foliated flat structure in question (i.e., the "geometric object" in question) can be extended to all of $\mathbb{C P}^{n}$. For example, considering the standard setting where the leaf $L$ is contained in $\mathbb{C}^{n}$, this leaf is endowed with a flat structure, or equivalently, with a (singular) Abelian form (called the time-form) induced by duality with the restriction of $X$ to $L$ itself. This Abelian form, however, does not extend to $\mathbb{C P}^{n}$ since $X$ has poles on the hyperplane at infinity $\Delta_{\infty}$ and the geometry arising from the mentioned Abelian form is not "bounded" in general. Nonetheless, the flat structure for which the above mentioned separating curve happens to be a geodesic does have an extension to $\Delta_{\infty}$; see Remark 6.3 in Section 6.1. Furthermore the "extended" flat structure still varies "continuously" with the leaves which, in turn, guarantees the existence of "bounds" for the corresponding geometry.

Now we return to the statement of Theorem A'. The fact that the preceding flat structure has "bounded geometry" implies, in particular, that Meas $\left(\mathcal{U}^{+} \cap B_{r}\right)$ is, in fact, comparable to the Euclidean measure of $B_{r}$ provided that $r$ is large. Since discs of large radius are also used in the construction of Ahlfors currents, the previous statement may look a bit surprising since these currents do not charge singular points. However, this difference is easily explained since the construction of Ahlfors currents is based on the "global volume" of a leaf and this may have little relation with the asymptotic behavior of the solution of the corresponding differential equation which is a function of a different parameter, namely the "time". To be more precise, fix a diffeomorphism between $\mathbb{C}$ and a leaf $L$, for example the time $t$ diffeomorphism $\Phi_{t}$ induced by the corresponding vector field. To construct the Ahlfors current, the ambient manifold is equipped with a Hermitian metric which 
is then pulled back by $\Phi_{t}$ to yield a metric $d_{\mathbb{C}}$ on $\mathbb{C}$. The desired current is then constructed by choosing a suitable sequence of discs $B_{r_{i}}$ whose radii $r_{i}$ are measured with respect to $d_{\mathbb{C}}$ and satisfy $r_{i} \rightarrow \infty$. Clearly a small neighborhood $V$ of a singular point in $M$ has small diameter for the fixed Hermitian metric and so does a connected component $(L \cap V)_{0}$ of $L \cap V$. The diameter (resp. the "area"), of $\phi_{t}^{-1}\left((L \cap V)_{0}\right)$ with respect to $d_{\mathbb{C}}$ is therefore small as well. Now, the metric $d_{\mathbb{C}}$ may differ markedly from the Euclidean metric on $\mathbb{C}$ so that the Euclidean area of $\phi_{t}^{-1}\left((L \cap V)_{0}\right)$ might be large. The proofs of the preceding theorems will make it clear that this phenomenon is precisely what happens in these cases. As a conclusion, whereas Ahlfors currents are among the most efficient tools for studying (singular) holomorphic foliations possessing leaves covered by $\mathbb{C}$, they might be less so when the main object of study is solution of a differential equation viewed as a function of the parameter "time".

The statements of Theorems A and A' indicate that the structure of the singularities of $\mathcal{D}$ lying in $\Delta_{\infty}$ must carry significant information about the global dynamics of corresponding vector fields. This can be thought of as a principle similar to Painlevé's test for the existence of meromorphic solutions for a differential equation (this is a context where our methods can also be applied). In a sense, this might partly explain the remarkable effectiveness of Painlevé's test. In any case, letting this principle guide us, it is natural to wonder that complete vector fields whose associated foliations $\mathcal{D}$ have only simple singularities in $\Delta_{\infty}$ must be amenable to a detailed global analysis. Throughout this paper, asimple singularity means one of the following types of singular points $q \in \Delta_{\infty}$ for $\mathcal{D}$ :

(1) Nondegenerate singularities: this means that $\mathcal{D}$ can locally be represented by a vector field having nondegenerate linear part at $q$ (i.e., the Jacobian matrix of $X$ at $q$ is invertible, equivalently, it possesses $n$ eigenvalues different from zero). Since resonances may arise, we assume that $q$ is not of Poincaré-Dulac type, i.e., if all the eigenvalues of $\mathcal{D}$ at $q$ belong to $\mathbb{R}_{+}^{*}$ then $\mathcal{D}$ must be locally linearizable at $q$.

(2) Codimension 1 saddle nodes: these are singularities of $\mathcal{D}$ lying in $\Delta_{\infty}$ whose eigenvalue associated to the direction transverse to $\Delta_{\infty}$ is equal to zero whereas it has $n-1$ eigenvalues different from zero and corresponding to directions contained in $\Delta_{\infty}$. Again we require that the $(n-1)$-dimensional singularity induced on the plane $\Delta_{\infty}$ should not be a singularity of PoincaréDulac type.

Note that singular points of $\mathcal{D}$ as in (1) above are necessarily isolated though this is no longer the case for Codimension 1 saddle nodes since these singularities may be contained in a curve of singularities of $X$ transverse to $\Delta_{\infty}$. Next we have:

Theorem B. Let $X$ be a complete polynomial vector field on $\mathbb{C}^{n}$ whose singular set has codimension at least 2. Suppose that all singularities of $\mathcal{D}$ lying in $\Delta_{\infty}$ are simple. Then $X$ has degree at most 1 .

Note that the assumption that $X$ is complete as vector field is essential for the preceding statement and cannot be replaced by other standard weaker notions 
such as the slightly weaker condition of having meromorphic solutions defined on all of $\mathbb{C}$. In fact, consider the pair of commuting vector fields given by

$$
\begin{aligned}
& Z_{0}=\left(-3 x^{2}+y^{2}+2 x z\right) \frac{\partial}{\partial x}+2 y(-3 x+2 z) \frac{\partial}{\partial y}+2 z(3 x-z) \frac{\partial}{\partial z} \\
& Z_{\infty}=2 y(-x+z) \frac{\partial}{\partial x}+\left(3 x^{2}-y^{2}\right) \frac{\partial}{\partial y}+2 y z \frac{\partial}{\partial z}
\end{aligned}
$$

Consider also the linear span of $Z_{0}$ and $Z_{\infty}$, i.e., all vector fields that are obtained as a linear combination of $Z_{0}$ and $Z_{\infty}$. It is shown in [24] that the solutions of every element in this family of vector fields are meromorphic functions defined on all of $\mathbb{C}$. In other words, these vector fields are very close to being complete vector fields. In addition, every two members of this family have essentially the same simple singularities on $\Delta_{\infty}$ and these are simple in the above indicated sense. Nonetheless, not only are vector fields in this family quadratic, but this family contains an infinite set of vector fields whose underlying foliations are not completely integrable in the sense that their leaves cannot be compactified into compact Riemann surfaces. This example, as well as the work of Guillot in [25] and [26], sheds light on the importance of the assumption on completeness made in the statement of Theorem B. Similarly, it also shows that the same completeness assumption is crucial for Theorems A and A' as well.

Theorem B will be proved in Section 6.2. The statement of this theorem may be compared to results of [15] for complete polynomial vector fields on $\mathbb{C}^{2}$. It is to be noted that the results of [15] chronologically preceded the classification obtained in [7]. Also, the more recent paper [29] contains a general classification theorem for meromorphic vector fields admitting maximal solutions on algebraic surfaces and these include complete vector fields as in [7]. All these questions are however wide open for $n \geq 3$ and Theorem $\mathrm{B}$ is a contribution to answering them.

To have a better appreciation of the difficulties involved in these problems, following [29], we consider the case of semi-complete vector fields, i.e., vector fields admitting solutions defined on maximal domains of $\mathbb{C}$. A vector field is said to be semi-complete on a domain $U$ if the solution $\phi$ satisfying $\phi(0)=p \in U$ of the associated differential equation is defined on a maximal domain of $\mathbb{C}$ for all $p \in U$. Here, a domain $V \subseteq \mathbb{C}$ where the solution $\phi$ is defined is said to be maximal if for every point $\hat{T}$ in the boundary $\partial V$ of $V$ and every sequence $\left\{T_{i}\right\} \subset V$ such that $T_{i} \rightarrow \hat{T}$, the sequence $\phi\left(T_{i}\right)$ leaves every compact set in $U$; see Section 2.2 for further details. Clearly, a complete vector field is automatically semi-complete since we can take $V=\mathbb{C}$ so that $\partial V=\emptyset$. If polynomial semi-complete vector fields on $\mathbb{C}^{n}$ are considered, then even the quadratic homogeneous case is already hard to understand once $n \geq 3$. A. Guillot has conducted detailed research about semi-complete quadratic homogeneous vector fields in [25] and [26]. In [25], by building on a certain variant of the Painlevé test, he introduced certain lattices (of coefficients) where all these vector fields are to be found, while in [26] he studied the special case of Halphen vector fields and the related problem about actions of PSL $(2, \mathbb{C})$ on compact 3 -manifolds. The beauty and depth of these results motivated us to try to apply our techniques to vector fields satisfying the conditions 
stated in [25] which will be said to belong to the Painlevé-Guillot lattice (the reader interested in the case $n=3$ is referred to [23] for an especially detailed discussion).

Note that a semi-complete vector field of $\mathbb{C}^{3}$ belonging to the Painlevé-Guillot lattice may not be complete and, moreover, its orbits (thought of as leaves of the associated foliation) may be hyperbolic Riemann surfaces. The latter situation actually occurs with Halphen vector fields except for a few special cases; see [23], [26], or Section 7.2. In this introduction, a Halphen vector field always means a hyperbolic Halphen vector field in the sense explained in Section 7.2. These vector fields are semi-complete and their solutions are defined on maximal domains which are either a bounded region of $\mathbb{C}$ or an unbounded hyperbolic region (for example the complement of a disc).

Among the various results obtained by Guillot on quadratic semi-complete vector fields (see [23], [25], [28]) there is a special class of exceptional cases whose dynamics is very hard to understand. In this direction, our methods allow us to say something nontrivial about these dynamics by considering the existence of dicritical singularities at infinity for the corresponding vector fields. Given a vector field $X$ in a Painlevé-Guillot lattice, a singular point for the associated foliation lying in the hyperplane at infinity which has all its eigenvalues contained in $\mathbb{R}_{+}$ will be called a dicritical singularity at infinity for $X$. Now we have:

Theorem C. Suppose that $X$ is a holomorphic vector field defined on a compact manifold $N$. Consider a singularity $p \in N$ of $X$ and denote by $X_{2}$ the first nonzero homogeneous component of the Taylor series of $X$ at $p$ (which is supposed to be quadratic). Suppose that one of the following condition holds:

- $\mathrm{X}_{2}$ is a vector field in the Painlevé-Guillot lattice having no dicritical singularity at infinity.

- $X_{2}$ is a hyperbolic Halphen vector field (in this case $N$ has dimension 3 ).

Then $N$ does not a carry a Kähler structure.

Note that the second item of Theorem $\mathrm{C}$ is sharp in the sense that [26] contains examples of compact 3-manifolds equipped with a global holomorphic vector field exhibiting the singularity of a hyperbolic Halphen vector field. Naturally the corresponding manifolds are not Kähler.

As to the first item, we are aware of no explicit example of vector field having no dicritical singularity at infinity and these do not exist for $n=2$. They are unlikely to exist for $n=3$, though we have no clear idea of what may happen in higher dimensions. In fact, from the known (low-dimensional) cases, it appears that the quadratic vector fields in question have a tendency to exhibit dicritical singularities at infinity. In this sense, as stated, the first item of Theorem $\mathrm{C}$ may be vacuous. However, there are several ways to turn this item into a meaningful statement about the dynamics of the vector field in question when dicritical singularities at infinity are present; see Section 2.2. For example, we have:

Theorem C'. Suppose that $X$ is a holomorphic vector field defined on a compact Kähler manifold $N$ of dimension $n$. Consider a singularity $p \in N$ of $X$ and denote by $\mathrm{X}_{2}$ the first nonzero homogeneous component of the Taylor series of $X$ at $p$. 
If $\mathrm{X}_{2}$ belongs to the Painlevé-Guillot lattice, then $X_{2}$ has dicritical singularities at infinity. Furthermore, every regular leaf of the foliation induced by $X_{2}$ on $\Delta_{\infty}$ must pass through one of these dicritical singular points.

Theorems C and C' will be proved in Section 7. The proofs are, very short and rely on the material developed in the preceding sections. The rest of Section 7 contains a discussion of the main dynamical issues associated to Halphen vector fields. The corresponding results are definitely not new as they can all be found in [26] together with a large amount of additional information. However, the discussion in Section 7 makes the article self-contained in the sense that all the properties of Halphen vector fields needed to prove Theorems C and C' are worked out here. Moreover, we have two additional motivations to carry out the mentioned analysis. Our first motivation has to do with the well-known fact that, in the context of differential equations without movable critical points, there is the phenomenon of natural boundaries. When this phenomenon is regarded from the point of view of semi-complete vector fields, it simply means that the maximal domain of definition of the solution is bounded in $\mathbb{C}$. This is precisely what happens in the case of Halphen vector fields. Whereas the methods used to prove Theorems $\mathrm{A}$ and $\mathrm{B}$ have natural reformulations in the case of solutions of differential equations that happen to be meromorphic functions on $\mathbb{C}$, it is unclear that these provide information in the case of solutions having a natural boundary. This leads us to work out the discussion of Halphen vector fields to show how the presence of an associated fibration can be combined with ideas from Kleinian group theory to yield new insights in these cases as well. A second motivation is that our discussion leads to a generalization of this picture in terms of representations of SL $(2, \mathbb{C})$ in higher dimensions. Indeed, the paper ends with an appendix containing some questions for which we believe the ideas developed here may be useful. These questions include nonfree representations of SL $(2, \mathbb{C})$.

Acknowledgements. Both authors are very grateful to A. Guillot for many discussions concerning several aspects of this work and, in particular, for having explained to us many issues in [26]. Thanks are also due to the anonymous referee for very valuable comments and suggestions. Discussions with X. GómezMont concerning complete real vector fields and Lorenz equations also improved our understanding of the material. Finally additional comments by F. Cano and J.-P. Ramis have helped to enlarge the scope of possible future applications of this work.

\section{Overview of methods, further results, and background ma- terial}

This section contains a general description of the structure of the paper as well as some qualitative explanation of our techniques. Quantitative information required by the corresponding proofs will be given in the subsequent sections. Some complements to the theorems stated in the introduction will also be provided along with background material on semi-complete vector fields. 


\subsection{Methods and results}

First, a general point should be made about the vector fields and differential equations considered in this work. This is due to the fact that they are far from being generic. For example, complete vector fields on $\mathbb{C}^{n}$ are very nongeneric among polynomial and rational vector fields or among singular holomorphic foliations on projective spaces. Indeed, as Riemann surfaces, the leaves of a foliation on $\mathbb{C P}^{n}$ induced by a complete vector field are quotients of $\mathbb{C}$ and this, by itself, is already very nongeneric. Whereas they are non-generic, they are interesting since, for example, they constitute a natural Lie algebra for the group of algebraic automorphisms of $\mathbb{C}^{n}[3]$ and remain an object of intensive study as shown by the recent works of A. Bustinduy, L. Giraldo, M. Brunella, and others (see [9], [10] and [7]). When working with differential equations, one often encounters very special (i.e., non-generic) examples that turn out to play crucial roles in the theory. Apart from complete vector fields, our techniques also apply to semi-complete ones, i.e., to those vector fields admitting solutions defined on maxima subsets of $\mathbb{C}$ (see below and Section 2.2 for further details). Halphen vector fields as studied in [26] satisfy this condition and they will be revisited in Section 7 . The importance of Halphen vector fields is undisputed since they appear in mathematical physics as well as in number theory through the celebrated functions $P, Q$ and $R$ of Ramanujan. Yet another class of special equations that fits in the pattern of our theory consists of those equations having meromorphic solutions defined on all of $\mathbb{C}$. Here the reader is reminded that the class of differential equations with meromorphic solutions includes the Painlevé 1, 2 and 4 equations, the modified Painlevé 3 and 5 equations as well as many Chazy equations. As already mentioned, linear integral equations in Gelfand-Levitan-Marcenko form also lead to equations having P-property that can be treated similarly; see [1], [2]. Even in the case when the solutions possess a natural boundary, and therefore are not defined on all of $\mathbb{C}$, our methods can sometimes be used. A concrete example of this situation is provided by our discussion of Halphen vector fields in Section 7. Another direction that is left for future investigation concerns the connections of our work with the point of view developed by X. Gómez-Mont and his collaborators concerning in particular the real Lorenz attractor for which a "real" variant of our method seems to yield new information; see [5] and [22].

Now we outline the structure of this paper. Consider a polynomial vector field $X$ on $\mathbb{C}^{n}$ and denote by $\mathcal{D}$ the associated foliation induced on $\mathbb{C P}^{n}$. Let $X_{d}$ stand for the top degree homogeneous component of $X$ (having degree $d \geq 2$ ) and suppose that $X_{d}$ is not a multiple of the radial vector field. Under this assumption, the foliation $\mathcal{D}$ leaves the hyperplane at infinity $\Delta_{\infty}=\mathbb{C} \mathbb{P}^{n} \backslash \mathbb{C}^{n}$ invariant. In addition, and modulo a minor issue discussed in Section 3 , the restriction of $\mathcal{D}$ to $\Delta_{\infty}$ coincides with the foliation induced on $\Delta_{\infty}$ by $X_{d}$. Alternatively, and modulo the natural identification $\Delta_{\infty} \simeq \mathbb{C P}^{n-1}$, the foliation in question is simply given by the direction of $X_{d}$ projected on $\mathbb{C P}^{n-1}$ viewed as the space of radial lines in $\mathbb{C}^{n}$ (note that $X_{d}$ is homogeneous and it is not a multiple of the radial vector field). A third way to see this foliation consists of noting that it coincides with the foliation induced on the exceptional divisor $\Delta_{0} \simeq \mathbb{C P}^{n-1}$ by the one point blow-up 
of $X_{d}$ at the origin. The foliation on $\mathbb{C P}^{n}$ associated to $X_{d}$ is denoted by $\mathcal{F}$ and its restriction to $\Delta_{\infty}$ is denoted by $\mathcal{F}_{\infty}$. If $L_{\infty}$ is a leaf of $\mathcal{F}_{\infty}$, then the "cone over $L_{\infty}$ " is invariant by $\mathcal{F}$.

Fundamentally our method relies on estimating the "speed" of the vector field $X$ near $\Delta_{\infty}$. This is done in two steps. The first step consists of eliminating the unbounded factor of $X$ over $\Delta_{\infty}$ so as to obtain a local regular vector field about every regular point $p \in \Delta_{\infty}$ of $\mathcal{F}_{\infty}$. However, it turns out that these locally defined vector fields depend to some extent on the choice of local coordinates so that they do not patch together to yield a foliated global vector field. Nonetheless, two local representatives obtained through overlapping coordinates differ only by a multiplicative constant. This means that this collection of local vector fields defines a global affine structure (induced by $X_{d}$ or by $X$ ) on every leaf of $\mathcal{F}_{\infty}$. In other words, the foliation $\mathcal{F}_{\infty}$ can be equipped with a global foliated affine structure although this affine structure does not give rise to a global foliated vector field. Another version of this affine structure already appeared in [25] as well as in a previous work of the first author [35] under the name of renormalized time-form. It also plays an important role in [29]. In our context, the interest of the mentioned affine structure is that it helps to provide estimates for the flow of $X$ as long as accurate estimates for the distance from the orbit in question to $\Delta_{\infty}$ are available.

Now we describe the second ingredient of our construction, namely a quantitative measure for the decay of the distance between a local piece of leaf of $\mathcal{F}$ and $\Delta_{\infty}$. Because $\Delta_{\infty} \subset \mathbb{C} \mathbb{P}^{n}$ and the Fubini-Study metric on $\mathbb{C P}^{n}$ has positive curvature, it is well known that complex submanifolds always bend towards $\Delta_{\infty}$; see for example [32]. In other words, the distance induced by the Fubini-Study metric between a leaf $L$ of $\mathcal{F}$ to $\Delta_{\infty}$ cannot have a local minimum at a point $p \in L$ unless $p$ also belongs to $\Delta_{\infty}$ (in which case the distance is zero). Our mentioned second ingredient is a consequence of this remark. Actually, we shall use the Euclidean metric in suitably chosen affine coordinates, as opposed to the globally defined Fubini-Study metric. The advantage of choosing this Euclidean metric lies in the fact that it is better adapted to working with the above mentioned affine structure. Besides, by exploiting the fact that the submanifolds in question are leaves of a foliation, an estimate for the rate of decay for the distance of a leaf to $\Delta_{\infty}$ is derived. The phenomenon is essentially as follows. At each regular point $p$ of a leaf $L$ of $\mathcal{F}$ there is the steepest descent direction of $L$ towards $\Delta_{\infty}$, namely the negative of the gradient of the distance function restricted to $L$. This yields a singular real one-dimensional oriented foliation $\mathcal{H}$ on $L$. Furthermore the conformal structure on $L$ is such that the foliation $\mathcal{H}^{\perp}$ orthogonal to $\mathcal{H}$ is constituted by level curves of the mentioned distance function.

Roughly speaking, it can then be obtained that $L$ approaches $\Delta_{\infty}$ exponentially fast along the trajectories of $\mathcal{H}$. Combined to the uniform estimates related to the foliated affine structure, this estimate yields accurate estimates for the time taken by the flow of $X$ along the trajectories of $\mathcal{H}$. The discussion actually shows that the time taken by $X$ to cover an entire (infinite) trajectory is finite provided that the trajectory remains away from the singularities of $\mathcal{F}$ lying in $\Delta_{\infty}$. This result is sharpened in Section 5 by allowing the trajectory to accumulate on simple singular 
points. An analogous estimate is still obtained. In particular, there is only one special type of simple singularity that can yield an "endpoint" for the trajectories of $\mathcal{H}$ and, in this case, this will be a point of intersection of the leaf $L$ and the hyperplane $\Delta_{\infty}$ : the corresponding trajectory of $\mathcal{H}$ should then be thought of as being finite. Finally, switching back and forth between estimates involving $X_{d}$ and estimates involving $X$ is not hard since $X$ is close to $X_{d}$ near $\Delta_{\infty}$.

The material mentioned above is covered in Sections 3, 4, and 5. Armed with these results we begin in Section 6 to prove the theorems stated in the introduction. Theorems A and A' are very natural. Since $X$ is complete, the integral of its timeform over a trajectory of $\mathcal{H}$ cannot converge (where the reader is reminded that the time-form is the Abelian dual to $X$ on the leaves of $\mathcal{D}$ ). Moreover this trajectory can never reach $\Delta_{\infty}$ since $X$ is complete on $\mathbb{C}^{n}$. This observation appears to clash with our previous estimate asserting convergence of the integral in question as long as the corresponding trajectory remains away from the singularities of $\mathcal{F}$ (or $\mathcal{D}$ ) lying in $\Delta_{\infty}$. The apparent contradiction is then explained by the fact that the flow of $X$ spends all but a finite amount of its existence in arbitrarily small neighborhoods of the singular set. The proof of Theorem A' goes along similar lines. In fact, the results obtained for the foliation $\mathcal{H}$ are also valid for every oriented foliation $\mathcal{H}^{\theta}$ forming an angle $\theta \in(-\pi / 2, \pi / 2)$ with $\mathcal{H}$. Once again the foliations $\mathcal{H}^{\theta}$ are well defined since the leaves of $\mathcal{F}, \mathcal{D}$ are endowed with conformal structures. Modulo fixing a base point and using the obvious identifications, the union of the corresponding trajectories spans an unbounded region of $\mathbb{C}$ viewed as the domain of definition of the solution in question. Finally, we shall also have control on the geometry of this region which will enable us to derive Theorem A'.

Given a solution $\phi: \mathbb{C} \rightarrow \mathbb{C}^{n}$ of a complete polynomial vector field, we say that $\phi$ is eventually confined at a singular point $p \in \Delta_{\infty}$ if for every neighborhood $U \subset \mathbb{C P}^{n}$ of $p$, there is a compact set $K \subset_{C}$ such that $\phi(T) \in U$ whenever $T \in_{C} \backslash K$. Theorems A and A' are clearly sharp a generic solution of a complete vector field cannot be eventually confined at singular points unless the vector field is completely integrable. Indeed, owing to the Remmert-Stein theorem, this type of confinement would mean that the solution is contained in a rational curve. In turn, if most solutions are contained in rational curves, then the underlying foliations must have all its leaves contained in rational curves which implies, in particular, that the vector field is completely integrable as desired. There are, however, simple examples complete polynomial vector fields on $\mathbb{C}^{2}$, such as $y \partial / \partial y+x y[x \partial / \partial x-$ $y \partial / \partial y]$, whose orbits accumulate on all of the line at infinity. These orbits are therefore Zariski dense.

In view of Theorems A and A', it is natural to imagine that the singular set of $\mathcal{D}$ contains significant information about the global geometry of complete polynomial vector fields. Theorem B is a contribution to the study of these vector fields as well as a test of the extent to which their global dynamics can be determined by the structure of their singularities. From an abstract point of view, this may be seen as a first attempt at understanding the remarkable effectiveness of the Painlevé test in differential equations. In fact, since Theorems A and A' can be adapted to the context of differential equations having meromorphic solutions, we can expect 
that the local information concentrated in the singular points is likely to strongly influence the global behavior of the solution itself. To substantiate this expectation, the idea will be to consider complete vector fields having simple singularities and to check what can then be said about the vector field in question. From this point of view, Theorem B is satisfactory since the dynamics of the corresponding vector field is fully determined.

The proof of Theorem B is arguably the most elaborate application of our techniques. Let us briefly describe its main ingredients. The central difficulty is to guarantee the existence of a "dicritical singularity" for $\mathcal{D}$ in $\Delta_{\infty}$, i.e., a linearizable singularity all of whose eigenvalues belong to $\mathbb{Z}_{+}$. The existence of this type of singularity implies, in particular, that the generic orbit of the vector field $X$ is of type $\mathbb{C}^{*}$ in the sense of [37] and several additional properties follow at once. To ensure the existence of this singularity is, however, a subtle question that can be approached as follows. First, let $X$ be replaced by its top degree homogeneous component $X_{d}$ along with its associated foliation $\mathcal{F}$. The property of having a dicritical singularity at $\Delta_{\infty}$ is common to $\mathcal{D}$ and $\mathcal{F}$ so that it is more convenient to work with homogeneous vector fields. Nonetheless, when replacing $X$ by $X_{d}$, we need to cope with the fact that $X_{d}$ is no longer complete but only semi-complete. In other words, every solution $\phi: V \subset \mathbb{C} \rightarrow U$ of $X_{d}$ on $\mathbb{C}^{n}$ is such that whenever a sequence $\left\{T_{i}\right\} \subset V$ converges to a point $\hat{T}$ in the boundary of $V$ the corresponding sequence $\phi\left(T_{i}\right)$ leaves every compact set in $U$. Being only semi-complete $X_{d}$ may reach infinity in finite time and this gives rise to further difficulties.

Another difficulty arising from the difference between semi-complete and complete vector fields is the fact that the leaves of the foliation associated to a semicomplete vector field may be hyperbolic Riemann surfaces, as happens in the case of Halphen vector fields; see Section 7. However, in the case of a foliation associated to the top degree homogeneous component of a complete vector field, it can be proved that the corresponding leaves are still quotients of $\mathbb{C}$. This is done by resorting to a result due to Brunella concerning the plurisubharmonic variation of the foliated Poincaré metric; see [6]. The solutions of $X_{d}$ will therefore be meromorphic functions defined on $\mathbb{C}$ or $\mathbb{C}$ minus one or two points. Next, we use our results involving the time taken by $X_{d}$ to cover trajectories of $\mathcal{H}$ in the singular context (namely the main result of Section 5, Theorem 5.1). Theorem 5.1 immediately implies that the solutions cannot be meromorphic on all of $\mathbb{C}$ and, by exploiting additional properties of the foliations $\mathcal{H}$ and $\mathcal{H}^{\perp}$, a contradiction ensuring the existence of the desired dicritical singularity is finally reached.

Let us now make some comments about the assumption that the singularities of $\mathcal{D}$ lying in $\Delta_{\infty}$ are simple in the sense described in the introduction. This assumption does not immediately simplify the problem since there may exist codimension 1 saddle nodes whose local analysis is already fairly complicated. Also, the statement of Theorem B may be extended to encompass more general singularities belonging to the class of absolutely isolated singularities; see [12]. While we shall not seek to establish any of these extensions, at the very end of Section 5 the reader will find some information on the structure of more degenerate singularities for which our methods might still work. It is also interesting to observe that our 
techniques apply equally well to rational vector fields as to polynomial ones. In practice, passing from polynomial to rational vector fields amounts to changing the divisor of poles of the vector field in question. The divisor of poles of a rational vector field may or may not include $\Delta_{\infty}$ and its analysis leads to numerous additional possibilities whose understanding may partially be facilitated by our ideas. In particular, several Painlevé equations are included in this class of problems.

As mentioned A. Bustinduy, L. Giraldo, and their collaborators have been investigating properties of the solutions of complete vector fields through various methods such as the theory of Nevanlinna, Andersen-Lempert theories, and so on; see [9], [10] and [3]. Similarly, if a classical result due to Forstneric [19] is taken into account, our method is likely to find some applications in the theory of holomorphic differential equations blowing up in finite real time. This should lead to some progress in questions similar to those treated by Fornaess and Grellier in [16] which itself connects with previous works by a number of authors including possible applications in the spirit of [11].

Finally, and as already mentioned, the beautiful results obtained by A. Guillot in [25] and [26] provide a natural motivation for trying to apply our techniques to quadratic semi-complete vector fields such as those considered by Guillot. These vector fields are referred to as belonging to the Painlevé-Guillot lattice. The tools developed in this paper will enable us to show that a vector field in the Painlevé Guillot lattice failing to have a dicritical singularity at infinity must have leaves that are hyperbolic Riemann surfaces. This fact, in turn, will quickly lead us to Theorem $\mathrm{C}$ by resorting again to Brunella's result on the variation of the Poincaré metric; see [6]. A point to be made here has to do with the lack of explicit examples of vector fields in the Painlevé-Guillot lattice having no dicritical singularity at infinity. We believe this example does not exist in dimension 3 and it is unclear to us whether or not it does in higher dimensions. However, the argument used in the proof of Theorem $\mathrm{C}$ allows us to conclude that the foliation induced on $\mathbb{C}^{3}$ associated to the vector field in question not only has a dicritical singularity at infinity, but also has the following properties:

- The foliation induced on the hyperplane at infinity is such that all its leaves have to pass through a dicritical singularity lying in the hyperplane in question (Theorem C').

- The restriction of the vector field $X$ to a generic leaf $L$ of its associated foliation is either complete or conjugate to the vector field $x^{2} \partial / \partial x$ on all of $\mathbb{C}$. In the second case, the blow-up of $X$ at the origin has a dicritical singularity on the exceptional divisor. Moreover, the saturate of this dicritical singularity by the foliation defines an open set where the vector field $X$ admits nonconstant first integrals.

Concerning the proofs of the above claims, the reader is referred to Remark 7.2.

Another minor point that can be mentioned is that the statement of Theorem $\mathrm{C}$ also holds for vector fields slightly more general than those belonging to the Painlevé-Guillot lattice. Namely, in our case, the condition used by Guillot may be relaxed to allow the eigenvalue associated to the direction transverse to the exceptional divisor to vanish. Another relatively minor point has to do with a slight 
relaxation of the assumption made in Theorems $\mathrm{C}$ and $\mathrm{C}^{\prime}$ or, more precisely, with the assumption that $X^{k}$ is quadratic, i.e., that $k=2$. Recall that in the statement of Theorems C and C' the vector field $X$ has the form $X=X_{k}+\cdots$ where $X_{k}$ is the first nonzero homogeneous component of the Taylor series of $X$ at the origin. Now, note that $X^{k}$ may have a codimension 1 zero set, in which case we can set $X_{k}=P . Y^{\mathrm{cd} 2}$ where $P$ is a homogeneous polynomial and $Y^{\mathrm{cd} 2}$ a homogeneous vector field whose zero set has codimension at least 2. Because the foliations induced on the corresponding projective space by $X^{k}$ and by $Y^{\mathrm{cd} 2}$ coincide, the statements of the mentioned theorems remain valid for vector fields $X$ whose first nonzero homogeneous component is a multiple of a semi-complete vector field lying in the Painlevé-Guillot lattice. In fact, in the Painlevé-Guillot lattice there are (semicomplete) vector fields admitting nonconstant holomorphic first integrals. If $Y$ stands for one of these vector fields and $P$ stands for a holomorphic first integral of $Y$, then the statement of Theorem $\mathrm{C}$ (resp. Theorem $\mathrm{C}$ ') also applies to vector fields $X$ whose first nonzero homogeneous component has the form $P Y$, for example. The reader will also notice that a similar extension concerning hyperbolic Halphen vector fields is vacuous in the sense that the vector fields in question have only constant holomorphic first integrals.

Among vector fields in the Painlevé-Guillot lattice, the examples provided by Halphen vector fields are again special in the sense that they do have dicritical singularities at infinity and still the leaves of their associated foliation may be hyperbolic Riemann surfaces. Although these results, and many others, are due to A. Guillot and appear in [26], we found it was worthwhile to rederive them by using our general point of view. This discussion takes up most of the last section of this paper. It involves, in particular, some considerations about convergence of Poincaré series that differ from the classical theory.

Another motivation for us to revisit Guillot's work on Halphen vector fields is to pave the way for additional possible applications of our techniques, some of them indicated in the appendix. These include the classification of the first homogeneous components at a singular point of a globally defined holomorphic vector field on a compact Kähler threefold. As will be explained later, this classification must be identical to the classification of the top degree homogeneous components of complete polynomial vector fields on $\mathbb{C}^{3}$; see the appendix.

\subsection{A brief review of semi-complete vector fields and additional back- ground material}

Most of the discussion below concerns basic properties of semi-complete vector fields that will often be encountered in the course of this paper. Some general subtle notions involving singular foliations and their corresponding leaves, as needed for Brunella's theorem [6], will also quickly be reviewed.

First consider a 1-dimensional singular holomorphic foliation $\mathcal{D}$ defined on a compact manifold $M$ and denote by $\operatorname{Sing}(\mathcal{D})$ its singular set. Thus $\operatorname{Sing}(\mathcal{D})$ is an analytic set of $M$ having codimension at least 2. Since Brunella's theorem [6] will be used in Section 6, we shall adopt the definition of leaf for $\mathcal{D}$ that is required by the statement of his theorem. The subtle point in this notion of leaf lies in the 
fact that leaves are sometimes allowed to contain points from $\operatorname{Sing}(\mathcal{D})$. Since the definition of leaf for the restriction of $\mathcal{D}$ to $M \backslash \operatorname{Sing}(\mathcal{D})$ is clear, we can work on a local setting and consider the $n$-dimensional polydisc $\mathbf{D}^{n}$ about the origin. This polydisc comes equipped with the trivial fibration $\mathbf{D}^{n}=\mathbf{D}^{n-1} \times \mathbf{D} \rightarrow \mathbf{D}^{n-1}$. A meromorphic map $f: \mathbf{D}^{n} \rightarrow M$ is said to be a foliated meromorphic immersion if the indeterminacy set $I(f)$ of $f$ intersects each vertical fiber of $\mathbf{D}^{n}$ in a discrete set and if $f$ satisfies the following additional conditions:

- $f$ is an immersion on the complement of $I(f)$.

- In the complement of $I(f), f$ takes vertical fibers to leaves of $\mathcal{D}$ (more generally to the leaves the foliation under consideration).

Consider now a regular point $p$ in $M \backslash \operatorname{Sing}(\mathcal{D})$ and let $L_{p}^{\prime}$ denote the leaf through $p$ of the (regular) foliation obtained by restriction of $\mathcal{D}$ to $M \backslash \operatorname{Sing}(\mathcal{D})$. A closed subset $K \subset L_{p}^{\prime}$ is called a vanishing end of $L_{p}^{\prime}$ if the following conditions are satisfied:

- $K$ is isomorphic to the punctured disc and the holonomy of the restriction of $\mathcal{D}$ to $M \backslash \operatorname{Sing}(\mathcal{D})$ corresponding to the cycle $\partial K$ has finite order $k$.

- There is a foliated meromorphic immersion $f: \mathbf{D}^{n} \rightarrow M$ such that

ı) $I(f) \cap(\{0\} \times \mathbf{D})=\{0\} \subset \mathbf{D} \subset \mathbb{C}$, where "0" stands for the origin of $\mathbf{D}^{n-1} \subset \mathbb{C}^{n-1}$.

ı) The image of $f$ restricted to $(\{0\} \times \mathbf{D})$ is the interior of $K$. Furthermore $f:(\{0\} \times \mathbf{D}) \rightarrow \operatorname{Int}(K)$ is a regular covering of degree $k$, where $\operatorname{Int}(K)$ stands for the interior of $K$.

The general definition of leaf of a foliation $\mathcal{D}$ on $M$ as above is as follows. Consider a regular point $p \in M \backslash \operatorname{Sing}(\mathcal{D})$ along with the leaf $L_{p}^{\prime}$ through $p$ of the (regular) foliation obtained by restricting $\mathcal{D}$ to $M \backslash \operatorname{Sing}(\mathcal{D})$. If $L_{p}^{\prime}$ possesses no vanishing ends, then the leaf $L_{p}$ of $\mathcal{D}$ containing $p$ is exactly $L_{p}^{\prime}$. Otherwise this leaf $L_{p}$ will consist of $L_{p}^{\prime}$ with the ends of the vanishing ends added to it where the operation of adding an end to $L_{p}^{\prime}$ should be understood in the sense of orbifolds; the multiplicity of the added point will be the order $k$ of the holonomy relative to $\partial K$. These orbifolds can then be turned into Riemann surfaces by the standard normalization. An immediate consequence of the preceding construction is the following.

Corollary 2.1. Let $\mathcal{D}, M$ and $\operatorname{Sing}(\mathcal{D})$ be as above. Fixed $p \in M \backslash \operatorname{Sing}(\mathcal{D})$, let $L_{p}\left(\right.$ resp. $\left.L_{p}^{\prime}\right)$ denote the leaf of $\mathcal{D}$ through $p$ (resp. the leaf of the restriction of $\mathcal{D}$ to $M \backslash \operatorname{Sing}(\mathcal{D})$ through $p)$. Then $L_{p}^{\prime} \subset L_{p}$ and $L_{p} \backslash L_{p}^{\prime}$ is a discrete set.

With the above definition of leaf, the main result of [6] reads as follows: if $\mathcal{D}$ is a singular holomorphic foliation defined on a compact Kähler manifold $M$, then the Poincaré metric along the leaves of $\mathcal{D}$ has plurisubharmonic variation. In particular, unless no leaf of the foliation in question is hyperbolic, the set of nonhyperbolic leaves is "small" in the sense that it is a pluripolar set. 
After these general considerations about holomorphic foliations, we recall that a meromorphic vector field $X$ defined on an open set $U$ of a (possibly open) manifold $M$ naturally defines a singular holomorphic foliation on $U$. In particular, if $X$ is a meromorphic vector field defined on a compact manifold $M$, then it induces a singular holomorphic foliation $\mathcal{D}$ on all of $M$.

Our next step is to recall the exact definition of semi-complete vector fields.

Definition 2.2. A holomorphic vector field $X$ on a complex manifold $M$ is called a semi-complete vector field if for every $p \in M$ there exists a connected domain $U_{p} \subset$ $\mathbb{C}$ with $0 \in U_{p}$ and a map $\phi_{p}: U_{p} \rightarrow M$ such that:

- $\phi_{p}(0)=p$ and $d \phi_{p}(t) /\left.d t\right|_{t=t_{0}}=X\left(\phi_{p}\left(t_{0}\right)\right)$.

- For every sequence $\left\{t_{i}\right\} \subset U_{p}$ such that $\lim _{i \rightarrow \infty} t_{i} \in \partial U_{p}$ the sequence $\left\{\phi_{p}\left(t_{i}\right)\right\}$ escapes from every compact subset of $M$.

A meromorphic vector field $X$ on a complex manifold $M$ is semi-complete if its restriction to the open set where $X$ is holomorphic is semi-complete in the above mentioned sense.

The reader will note that the standard theorem about existence of local solutions for ordinary differential equations ensures that a map $\phi: U_{p} \rightarrow M$ satisfying the first condition in the preceding definition always exists. It is therefore the second condition that makes the definition nontrivial. This second condition is a natural generalization of the analogous phenomenon that always happens for real time ordinary differential equations when the time approaches one of the endpoints of its maximal interval of definition. In this sense, semi-complete vector fields are those whose solutions admit a maximal domain of definition in $\mathbb{C}$. It follows at once from this definition that vector fields whose solutions are meromorphic functions defined on $\mathbb{C}$ are automatically semi-complete. Moreover, the solutions of semicomplete vector fields may actually be defined on bounded domains of $\mathbb{C}$ in an essential way; see [26] or Section 7. This essential boundary is thus a continuum of singularities for the solution of the associated differential equation. Furthermore, this boundary may move with the initial condition (or rather with the leaf of the underlying foliation). Thus this class of vector fields (or equations) is, in a sense, more general than those possessing Painlevé property; see, for example, [31].

The following simple lemma already conveys some useful information concerning semi-complete vector fields.

Lemma 2.3. A semi-complete meromorphic vector field on a curve is necessarily holomorphic.

Proof. Let $X$ be a meromorphic vector field on the curve $\Sigma$ and suppose that $X$ has a pole at $p \in \Sigma$. The vector field is given, in a neighborhood of $p$, by $z^{-q} f(z) \partial / \partial z$ for some $q>0$ and a nonvanishing holomorphic function $f$. There is a coordinate $w$ where the vector field has the form $w^{-p} \partial / \partial w$. The solution with initial condition $w_{0} \neq 0$ is multivalued and given by $\sqrt[p+1]{(1+p) t+w_{0}^{p+1}}$. Hence, there is no neighborhood of $p$ where the vector field is semi-complete. 
This implies that a semi-complete meromorphic vector field on a compact curve is globally holomorphic and thus, unless it is identically zero, the curve must be either elliptic or rational.

More generally, consider a meromorphic vector field $X$ along with its associated singular holomorphic foliation $\mathcal{D}$. The singular set of $\mathcal{D}$ (resp. $X$ ) will be denoted by $\operatorname{Sing}(\mathcal{D})$ (resp. $\operatorname{Sing}(X)$ ). Note that, unlike $\operatorname{Sing}(\mathcal{D})$, $\operatorname{Sing}(X)$ contains the divisor of zeros and poles of $X$ so that it can have codimension 1 components. Given a point $p \in M$ that is regular for $X$, consider the leaf $L_{p}$ of $\mathcal{D}$ through $p$. Inside $L_{p}$, there are two open sets that may naturally be considered, namely:

- The set $V_{\text {reg }} \subset L_{p}$. This set is identified with the leaf $L_{p}^{\prime}$ of the restriction of $\mathcal{D}$ to the complement of $\operatorname{Sing}(\mathcal{D})$.

- The set $W_{X}$ consisting of those points in $L_{p}$ at which the vector field $X$ is holomorphic and different from zero.

Clearly $W_{X} \subseteq V_{\text {reg }} \subseteq L_{p}$. On $W_{X}$, consider the time-form induced by $X$ namely, the 1-form $d T$ defined by letting $d T . X(q)=1$. The time-form is holomorphic and nonzero on $W_{X}$. It has a meromorphic extension to $V_{\text {reg }}$ and, a priori, may have essential singularities at the discrete set $L_{p} \backslash V_{\text {reg. }}$.

At this point two additional remarks can be made concerning the definition of semi-complete vector fields. The first one is that $X$ is semi-complete if and only if for every point $p$ regular for $X$, the natural map $\phi_{p}: U_{p} \rightarrow W_{X}$ is proper and, hence, a covering (since it is clearly a local diffeomorphism). Also, by exploiting this condition, it is easy to see that for every embedded (one-to-one) path $c:[0,1] \rightarrow W_{X} \subset L_{p}$, the integral

$$
\int_{c} d T
$$

is different from zero provided that $X$ is semi-complete; see [34].

At this point, Lemma 2.3 can be improved as follows.

Lemma 2.4. Consider a meromorphic vector field along with its associated singular foliation $\mathcal{D}$. Fix a leaf $L$ of $\mathcal{D}$ not contained in the divisor of zeros or poles of $X$ and suppose that $X$ is semi-complete. Then the restriction $X_{\left.\right|_{L}}$ of $X$ to $L$ is holomorphic on all of $L$. Besides, if $p \in L$ is a singular point of $X_{\left.\right|_{L}}$, then the second jet of $X_{\left.\right|_{L}}$ at $p$ is different from zero.

Proof. Let us first show that $X_{\left.\right|_{L}}$ is holomorphic. In view of Lemma 2.3, it suffices to show that $X_{\left.\right|_{L}}$ cannot have an essential singularity at a point $p \in L \backslash V_{\text {reg. }}$. Assuming, aiming at a contradiction, the existence of a point $p \in L \backslash V_{\text {reg }}$ at which $X$ has an essential singularity, note that $p$ also yields an essential singularity for the time-form $d T$ induced on $L$ by $X$. Now, fix a local disc $B \subset L$ about $p$ and consider the map Dev $: \widetilde{B \backslash\{p\}} \longrightarrow \mathbb{C}$ defined by

$$
\operatorname{Dev}(x)=\int_{x_{0}}^{x} d T,
$$

where $\widetilde{B \backslash\{p\}}$ stands for the universal covering of the punctured disc $B \backslash\{p\}$ and where $x_{0}$ is a fixed base point. The semi-completeness of $X$ implies that the 
map Dev must be one-to-one. This is however impossible as follows from a simple application of Picard theorem, see [35].

It remains only to check that the second jet of $X_{\left.\right|_{L}}$ cannot vanish at a (necessarily isolated) singular point. The proof is a simple variant of the argument given in the proof of Lemma 2.3. Details are left to the reader.

Lemma 2.4 has the following useful corollary.

Corollary 2.5. Suppose that $X$ is a semi-complete vector field defined on the complement of a discrete set $\aleph \subset \mathbb{C}$. Then $X$ is holomorphic on all of $\mathbb{C}$ and, in fact, extends to a holomorphic vector field globally defined on $\mathbb{C P}^{1}$.

Semi-complete vector fields have additional useful global properties. For example, unlike complete vector fields, semi-complete vector fields are invariant under birational transformations. In this sense, from the point of view of birational geometry, the notion of semi-complete vector field is more natural than the notion of complete vector field; see [29].

Another less immediate, though still elementary, global property originally established in [21] asserts that the space of semi-complete holomorphic vector fields is closed in the topology of uniform convergence. More precisely, suppose that $\left\{X_{n}\right\}$ is a sequence of holomorphic vector fields defined on some (possibly open) manifold $M$ converging to a (holomorphic) vector field $X$ on $M$ for the topology of uniform convergence on compact subsets. Under this assumption, the limit vector field $X$ must be semi-complete provided that $X_{n}$ is a semi-complete vector field for every $n \in \mathbb{N}$.

From the preceding results, the following useful fact can be derived.

Lemma 2.6. Suppose that $X$ is a semi-complete polynomial vector field on $\mathbb{C}^{n}$ having degree $d$. If $X_{d}$ denotes the homogeneous component of degree $d$ of $X$, then $X_{d}$ is itself semi-complete on all of $\mathbb{C}^{n}$. In particular, if $X_{d}$ is a nonconstant multiple $f R$ of the radial vector field $R=x \partial / \partial x+y \partial / \partial y+z \partial / \partial z$, then the degree of the (homogeneous) form $f$ must equal 1.

Proof. To show that $X_{d}$ is itself a homogeneous semi-complete vector field, consider the homothety $\Lambda^{k}$ of $\mathbb{C}^{n}$ having the form $\Lambda^{k}\left(x_{1}, \ldots, x_{n}\right)=\left(k x_{1}, \ldots, k x_{n}\right)$, for $k \in \mathbb{N}^{*}$. Clearly for every $k \in \mathbb{N}^{*}$, the vector field $\left(\Lambda^{k}\right)^{*} X$ is semi-complete on $\mathbb{C}^{n}$. Since a constant multiple of a semi-complete vector field is again semi-complete, it follows that the vector fields $Y_{k}=k^{1-d}\left(\Lambda^{k}\right)^{*} X$ are semi-complete on $\mathbb{C}^{n}$ for every $k \in \mathbb{N}$. When $k \rightarrow \infty$, it is clear that the sequence of vector fields $Y_{k}$ converges uniformly to $X_{d}$ on compact subsets of $\mathbb{C}^{n}$. It then follows that $X_{d}$ is semi-complete as desired.

For the second part of the statement, note that every radial line through the origin is left invariant by the radial vector field $R$ and hence by $X_{d}$. By restricting $X_{d}$ to a "generic" line as before, we obtain a 1-dimensional semi-complete vector field having an isolated singular point at the origin. However, owing to Lemma 2.4, the order of this singular point cannot exceed 2. Hence the degree of the nonconstant homogeneous polynomial $f$ cannot exceed 1 which completes the proof of the lemma. 


\section{Homogeneous vector fields and their foliations}

Unless otherwise stated, throughout this paper homogeneous vector fields are supposed to have degree $d \geq 2$ and not to equal a multiple of the radial vector field. In this section we work in dimension $n=3$ merely for notational simplicity, since all the arguments presented in the sequel carry over word-for-word to higher dimensions.

Consider a homogeneous polynomial vector field $X$ of degree $d \geq 2$ defined on $\mathbb{C}^{3}$. Since $X$ is homogeneous, its associated foliation $\mathcal{F}$ is invariant under homotheties of the form $(x, y, z) \mapsto(\lambda x, \lambda y, \lambda z), \lambda \in \mathbb{C}^{*}$, and, therefore, also induces a foliation of $\mathbb{C P}^{2}$. An alternative way to look at this situation consists of blowing-up $X$ at the origin of $\mathbb{C}^{3}$ (an one-point blow-up). We denote by $\widetilde{\mathbb{C}}^{3}$ the corresponding blow-up of $\mathbb{C}^{3}$ and by $\Delta_{0}=\pi^{-1}(0)$ the resulting exceptional divisor, where $\pi: \widetilde{\mathbb{C}}^{3} \mapsto \mathbb{C}^{3}$ is the corresponding projection. The transform (i.e., the blowup) $\widetilde{X}$ (resp. $\widetilde{\mathcal{F}}$ ) of $X$ (resp. $\mathcal{F}$ ) vanishes identically over $\Delta_{0}$ (resp. leaves $\Delta_{0}$ invariant), as follows from the fact that the degree of $X$ is strictly greater than 1 (resp. that $X$ is not a multiple of the radial vector field).

Recalling also that $\widetilde{\mathbb{C}}^{3}$ can be viewed as a line bundle over $\Delta_{0}=\pi^{-1}(0)$, let $\mathcal{P}_{0}$ denote the bundle projection $\mathcal{P}_{0}: \widetilde{\mathbb{C}}^{3} \rightarrow \Delta_{0}$. This line bundle can be compactified into a projective line bundle by adding the section at infinity $\Delta_{\infty}$. Denoting by $M$ the total space of the resulting projective line bundle, it follows that $M$ is equipped with two bundle projections $\mathcal{P}_{0}$ and $\mathcal{P}_{\infty}$ realizing it as a projective bundle respectively over $\Delta_{0}$ and $\Delta_{\infty}$. The manifold $M$ is also isomorphic to the blow-up of $\mathbb{C P}^{3}$ at the origin. The vector field $\widetilde{X}$ can be extended to $M$ as a meromorphic vector field; in particular it induces a holomorphic foliation, still denoted by $\widetilde{\mathcal{F}}$, on all of $M$. In addition, $\widetilde{\mathcal{F}}$ leaves both $\Delta_{0}$ and $\Delta_{\infty}$ invariant since $X$ is homogeneous and it is not a multiple of the radial vector field. The foliation induced on $\Delta_{0}$ (resp. $\Delta_{\infty}$ ) by restriction of $\widetilde{\mathcal{F}}$ is denoted by $\widetilde{\mathcal{F}}_{0}$ (resp. $\widetilde{\mathcal{F}}_{\infty}$ ). Because $\widetilde{\mathcal{F}}$ comes from a homogeneous vector field, these foliations coincide with the restrictions of $\widetilde{\mathcal{F}}$ to $\Delta_{0}$ and $\Delta_{\infty}$. As to the vector field $\widetilde{X}$, its pole divisor coincides with $\Delta_{\infty}$ and it has order $d-1>0$. The zero divisor of $\widetilde{X}$ is the union of $\Delta_{0}$ (a component of order $d-1>0$ ) with the transform of the zero divisor of $X$.

Naturally the singular set of $\widetilde{\mathcal{F}}$ has codimension at least 2. Furthermore this singular set is saturated (i.e., invariant) by the fibers of $\mathcal{P}_{0}$ (resp. $\left.\mathcal{P}_{\infty}\right)$ due to the invariance of $\mathcal{F}$ under homotheties of the form $(x, y, z) \mapsto(\lambda x, \lambda y, \lambda z), \lambda \in \mathbb{C}^{*}$. In particular, the foliations $\widetilde{\mathcal{F}}_{0}$ and $\widetilde{\mathcal{F}}_{\infty}$ automatically have singular sets of codimension at least 2 inside $\Delta_{0}$ and $\Delta_{\infty}$ (in other words the intersections of the singular set of $\widetilde{\mathcal{F}}$ with $\widetilde{\mathcal{F}}_{0}$ and with $\widetilde{\mathcal{F}}_{\infty}$ yield a set of codimension at least 2 inside $\Delta_{0}$ and $\left.\Delta_{\infty}\right)$.

Consider a nonalgebraic leaf $L$ of $\widetilde{\mathcal{F}}$ not contained in $\Delta_{0} \cup \Delta_{\infty}$. The projection of $L$ onto $\Delta_{0}\left(\right.$ resp. $\left.\Delta_{\infty}\right), \mathcal{P}_{0}(L)=L_{0}\left(\right.$ resp. $\left.\mathcal{P}_{\infty}(L)=L_{\infty}\right)$, is clearly a leaf of $\widetilde{\mathcal{F}}_{0}$ (resp. $\widetilde{\mathcal{F}}_{\infty}$ ) since the initial vector field $X$ is homogeneous. Furthermore one immediately checks that the restriction of $\mathcal{P}_{0}$ (resp. $\left.\mathcal{P}_{\infty}\right)$ to $L$ realizes $L$ as an Abelian covering of $L_{0}$ (resp. $\left.L_{\infty}\right)$. It then follows that the noncompact 
leaves $L, L_{0}$ and $L_{\infty}$ have all the same nature: either they are all covered by $\mathbb{C}$ or they are all covered by the unit disc $D$. Furthermore $L_{0}$ and $L_{\infty}$ are isomorphic as Riemann surfaces while $L$ is an Abelian covering of $L_{0}$ or of $L_{\infty}$.

Therefore, we may focus on the behavior of $\widetilde{X}$ near its pole divisor $\Delta_{\infty}$ or near $\Delta_{0}$, according to our convenience. Next, consider a leaf $L_{\infty}$ of $\widetilde{\mathcal{F}}_{\infty}$. By the cone over $L_{\infty}$ is meant the 2-dimensional immersed singular surface $\mathcal{P}_{\infty}^{-1}\left(L_{\infty}\right)$ which is invariant under $\widetilde{\mathcal{F}}$. In other words, if $\psi(T)=(x(T), y(T), 0), T \in \Omega \subseteq \mathbb{C}$, is a local parametrization of $L_{\infty}$, then the cone is parameterized by $\Phi(T, z)=$ $(x(T), y(T), z), z \in \mathbb{C}$. The singular points of $\mathcal{P}_{\infty}^{-1}\left(L_{\infty}\right)$ belong to fibers sitting over the singular set of $\widetilde{\mathcal{F}}_{\infty}$ which, we recall, may intersect $L_{\infty}$ nontrivially due to the definition of regular leaf adopted in Section 2.2. Away from its singularities, $\mathcal{P}_{\infty}^{-1}\left(L_{\infty}\right)$ can be viewed as a complex surface equipped with a singular holomorphic foliation. We denote this surface by $S$ and by $\widetilde{\mathcal{F}}_{S}$ the foliation on $S$ obtained by restricting $\widetilde{\mathcal{F}}$ to $S$. Note that $S$ is invariant under the automorphism $(x, y, z) \mapsto$ $(x, y, \lambda z), \lambda \in \mathbb{C}^{*}$, and so is the foliation $\widetilde{\mathcal{F}}_{S}$.

Since $S$ is a 2 -dimensional variety, $\widetilde{\mathcal{F}}_{S}$ is a codimension 1 singular foliation of $S$ and, hence, it has a transversely conformal structure. This yields good control of the directions over which the leaves of $\widetilde{\mathcal{F}}_{S}$ cluster together with respect to a suitably chosen auxiliary Hermitian metric. This idea is well known and can be found, for instance, in [20]. In our case, however, we shall use an explicit parametrization. For this, let $M$ be equipped with affine coordinates $(x, y, z)$ such that

(i) $\{z=0\} \subset \Delta_{\infty},(x, y) \in \mathbb{C}^{2}, z \in \mathbb{C}$;

(ii) the vector field $\tilde{X}$ is given by

$$
\widetilde{X}=\frac{1}{z^{d-1}}\left[F(x, y) \frac{\partial}{\partial x}+G(x, y) \frac{\partial}{\partial y}+z H(x, y) \frac{\partial}{\partial z}\right]
$$

where $F$ and $G$ are polynomials of degree either $d$ or $d+1$ and $H$ is a polynomial of degree $d$ (the fact that $F, G$ and $H$ do not depend on the variable $z$ is a consequence of the homogeneous character of $X$ );

(iii) The projection $\mathcal{P}_{\infty}: M \rightarrow \Delta_{\infty}$ in the above coordinates becomes $(x, y, z) \mapsto$ $(x, y)$.

Affine coordinates with the above indicated properties can be obtained as follows. Recall that the blow-up $\widetilde{\mathbb{C}}^{3}$ of $\mathbb{C}^{3}$ at the origin possesses affine coordinates $(x, y, w),(u, a, b)$, and $(r, v, s)$ arising from the realization of $\widetilde{\mathbb{C}}^{3}$ as the gluing of three copies of $\mathbb{C}^{3}$ by means of the identification

$$
\begin{aligned}
& b=\frac{1}{x}, u=x w \quad(b \neq 0, x \neq 0) \\
& a=\frac{1}{r}, u=r v \quad(a \neq 0, r \neq 0) \\
& s=\frac{1}{y}, v=y w \quad(s \neq 0, y \neq 0) .
\end{aligned}
$$


In the affine coordinates $(x, y, w)$, the vector field $\widetilde{X}$ takes the form

$$
\widetilde{X}=w^{d-1}\left[F(x, y) \frac{\partial}{\partial x}+G(x, y) \frac{\partial}{\partial y}+w H(x, y) \frac{\partial}{\partial w}\right]
$$

for some polynomials $F, G$, and $H$ depending only on the variables $x$ and $y$ (since $X$ is homogeneous). Now, to obtain the mentioned coordinates, it suffices to take $w=1 / z$.

Note that $\Delta_{\infty}$ is itself isomorphic to $\mathbb{C P}^{2}$. Thus the affine coordinates $(x, y) \simeq$ $(x, y, 0)$ on $\Delta_{\infty}$ defines an affine copy of $\mathbb{C}^{2}$ inside $\Delta_{\infty}$. Associated to the mentioned affine $\mathbb{C}^{2} \subset \Delta_{\infty}$, there is a notion of line at infinity for $\Delta_{\infty}$ itself. We shall denote this line by $\Delta_{\infty}^{(x, y)}$. In particular, it follows that the domain of definition of the coordinates $(x, y, z)$ coincides with the open set $M \backslash\left(\Delta_{0} \cup \mathcal{P}_{\infty}^{-1}\left(\Delta_{\infty}^{(x, y)}\right)\right)$. Naturally the choices of the affine coordinates $(x, y)$ and of the line $\Delta_{\infty}^{(x, y)}$ are not canonical. For a generic choice of these coordinates, $\Delta_{\infty}^{(x, y)}$ does not contain singular points of the corresponding foliation on $\Delta_{\infty}$ and $\Delta_{\infty}^{(x, y)}$ is not invariant by this foliation. Now, we have:

Lemma 3.1. Suppose that the affine coordinates $(x, y)$ are chosen so that the resulting line at infinity $\Delta_{\infty}^{(x, y)}$ is not invariant under the corresponding foliation on $\Delta_{\infty}$. Then the top degree component of the vector field $\widetilde{X}$ has the form

$$
z^{1-d} f(x, y)\left[x \frac{\partial}{\partial x}+y \frac{\partial}{\partial y}+z \frac{\partial}{\partial z}\right]
$$

for a certain homogeneous polynomial $f$ having degree $d$.

Proof. Suppose that the initial homogeneous vector field $X$ is given in the standard coordinates $\left(z_{1}, z_{2}, z_{3}\right)$ on $\mathbb{C}^{3}$ by $X=A\left(z_{1}, z_{2}, z_{3}\right) \partial / \partial z_{1}+B\left(z_{1}, z_{2}, z_{3}\right) \partial / \partial z_{2}+$ $C\left(z_{1}, z_{2}, z_{3}\right) \partial / \partial z_{3}$. Then, with the change of coordinates

$$
(x, y, z) \mapsto\left(\frac{x}{z}, \frac{y}{z}, \frac{1}{z}\right)=\left(z_{1}, z_{2}, z_{3}\right)
$$

the vector field $\widetilde{X}$ is given in a neighborhood of the hyperplane at infinity by

$$
\widetilde{X}=z^{1-d}\left[F(x, y) \frac{\partial}{\partial x}+G(x, y) \frac{\partial}{\partial y}+z H(x, y) \frac{\partial}{\partial z}\right]
$$

where $F(x, y)=A(x, y, 1)-x C(x, y, 1), G(x, y)=B(x, y, 1)-y C(x, y, 1)$ and $H(x, y)=-C(x, y, 1)$. Now, the initial Euclidean coordinates $\left(z_{1}, z_{2}, z_{3}\right)$ for $\mathbb{C}^{3}$ can be chosen so that none of the functions $A, B$, or $C$ is divisible by $z_{3}$. This assumption, combined with the noninvariance of the line at infinity $\Delta_{\infty}^{(x, y)}$ under the foliation in question, implies that $F$ and $G$ (resp. $H$ ) have degree $d+1$ (resp. $d$ ). Since $A(x, y, 1)$ and $B(x, y, 1)$ have degree at most $d$, it follows that the top-degree homogeneous component of $F$ (resp. $G$ and $H$ ) is given by $x$ (resp. $y$ and $z$ ) times the top degree homogeneous component of $C$. In other words, the top degree homogeneous component of the vector field $\widetilde{X}$ has the form (3.2) as desired. 
A further comment concerning the difference between the foliation $\widetilde{\mathcal{F}}_{\infty}$ induced by $\widetilde{X}$ on $\Delta_{\infty}$ and the corresponding foliation $\widetilde{\mathcal{F}}$ in 3 -dimensional space is also needed. To be more precise, consider the vector field $\widetilde{X}$ given by the formula (3.1) in the coordinates $(x, y, z)$. If $F$ and $G$ have only trivial common factors, then the foliation induced by $\widetilde{X}$ on $\Delta_{\infty}$ is given in $(x, y,\{z=0\})$ coordinates by $F(x, y) \partial / \partial x+G(x, y) \partial / \partial y$. Suppose now that $F$ and $G$ possess nontrivial common factors. Set $\mathrm{P}=\operatorname{gcd} .(F, G)$ so that $F=\mathrm{P} . a(x, y)$ and $G=\mathrm{P} . b(x, y)$ with $a$ and $b$ having only trivial common factors. In this case, the foliation $\widetilde{\mathcal{F}}_{\infty}$ is represented by the vector field $a(x, y) \partial / \partial x+b(x, y) \partial / \partial y$. With this observation in place, we need to go one step further and consider also the common divisors of $\mathrm{P}$ and $H$. When $\mathrm{P}$ and $H$ have nontrivial common factors, then these common factors can be (factored out and) eliminated without changing the foliations $\widetilde{\mathcal{F}}$ and $\widetilde{\mathcal{F}}_{\infty}$. Hence, as far as the foliations $\widetilde{\mathcal{F}}$ and $\widetilde{\mathcal{F}}_{\infty}$ are concerned, we can suppose without loss of generality that $\operatorname{gcd}(\mathrm{P}, H)$ is invertible. Once this normalization has been made, two distinguished cases may occur, namely:

- Suppose that $\mathrm{P}$ is invertible (after reducing to the case where $\operatorname{gcd}(\mathrm{P}, H)$ is invertible). Then the restriction of $\widetilde{\mathcal{F}}$ to $\Delta_{\infty}$ coincides with $\widetilde{\mathcal{F}}_{\infty}$. Moreover, in this case, the singular set of $\widetilde{\mathcal{F}}$ intersects $\Delta_{\infty}$ in finitely many points.

- Suppose that $\mathrm{P}$ is not invertible (after reducing to the case where gcd $(\mathrm{P}, H)$ is invertible). In this case, the foliation $\widetilde{\mathcal{F}}_{\infty}$ does not coincide with the restriction of $\widetilde{\mathcal{F}}$ to $\Delta_{\infty}$ since the latter contains a curve of singularities which is induced in the above coordinates by $\mathrm{P}$. In particular, the singular set of $\widetilde{\mathcal{F}}$ intersects $\Delta_{\infty}$ in a curve plus, occasionally, finitely many isolated points.

Summarizing the preceding discussion, the foliation $\widetilde{\mathcal{F}}$ associated to $\widetilde{X}$ can be supposed to be given by a polynomial vector field of the form

$$
Y=\mathrm{P}\left[a(x, y) \frac{\partial}{\partial x}+b(x, y) \frac{\partial}{\partial y}\right]+z H(x, y) \frac{\partial}{\partial z},
$$

where $\operatorname{gcd}(\mathrm{P}, H)$ is constant. Furthermore the previously defined vector field $\widetilde{X}$ is given in the same coordinates by

$$
\widetilde{X}=z^{1-d} Q(x, y) Y,
$$

where $Q(x, y)$ is a polynomial. From this, it also follows that the projective curve $\{\mathrm{P}=0\} \subset \Delta_{\infty}$ (if not empty) is constituted by singularities of $\widetilde{\mathcal{F}}$ whereas its "generic" point is regular for $\widetilde{\mathcal{F}}_{\infty}$. Furthermore there are two different possibilities that need to be considered:

(a) $\{\mathrm{P}=0\} \subset \Delta_{\infty}$ is invariant under $\widetilde{\mathcal{F}}_{\infty}$.

(b) $\{\mathrm{P}=0\} \subset \Delta_{\infty}$ is not invariant under $\widetilde{\mathcal{F}}_{\infty}$.

Remark 3.2. It will be seen later (Propositions 4.1 and 4.2$)$ that $\{P=0\}\left(\subseteq \Delta_{\infty}\right)$ is not invariant under $\widetilde{\mathcal{F}}_{\infty}$ provided that the homogeneous polynomial vector field of degree $d \geq 2$ is supposed also to be semi-complete. For this reason the possibility of having $\{P=0\}$ invariant under $\widetilde{\mathcal{F}}_{\infty}$ will be excluded from our discussion. 
Our purpose is now to equip the leaves of $\widetilde{\mathcal{F}}$ in $\Delta_{\infty}$ with an Abelian form $\omega_{1}$ naturally related to the holonomy of the leaf in question. This will be done in the affine copy of $\mathbb{C}^{3}$ in $M$ corresponding to the domain of definition of the coordinates $(x, y, z)$. With the preceding notations, we fix a regular leaf $L_{\infty} \subset \Delta_{\infty}$ and a point $p \in L_{\infty}$ regular for $\widetilde{\mathcal{F}}$. Under this assumption, the leaf $L_{\infty}$ can locally be parametrized in the form $(x, y(x))$ or in the form $(x(y), y)$, with $z=0$. It suffices to consider a local parametrization of the form $(x, y(x))$ since the other possibility is analogous. The vector field $\widetilde{X}$ then yields

$$
d z / d x=z H(x, y(x)) / F(x, y(x)) .
$$

Therefore

$$
z=z_{0} \exp \left[\int_{x_{0}}^{x} \frac{H(x, y(x))}{F(x, y(x))} d x\right] .
$$

Thus we define an Abelian form $\omega_{1}$ on $L_{\infty}$ by declaring that the coefficient of $\omega_{1}$ at $(x, y(x))$ is nothing but $-H(x, y(x)) / F(x, y(x))$ (the minus sign is only a matter of convention). In particular we note that possible nontrivial common factors of $F$ and $H$ are automatically canceled out in the definition of $\omega_{1}$. If the leaf were parameterized in the form $(x(y), y)$, the analogous result would yield for the coefficient $-H(x(y), y) / G(x(y), y)$. The form $\omega_{1}$ is the restriction of the form $d z / z$ to the leaf in question. This form can also be interpreted as the logarithmic derivative of the holonomy for the foliation $\underset{\sim}{\widetilde{\mathcal{F}}_{S}}$ induced on the cone $S$ over $L_{\infty}$. This means the following: let $L$ be a leaf of $\widetilde{\mathcal{F}}_{S}$ and consider a path $c:[0,1] \mapsto L$, on $L$. Denoting by $\operatorname{Hol}(c)$ the holonomy associated to $c$, we have

$$
(\operatorname{Hol}(c))^{\prime}(c(0))=e^{-\int_{c} \omega_{1}},
$$

where $\operatorname{Hol}(c)$ is identified with a map between open sets of $\mathbb{C}$ equipped with the coordinate $z$.

Once a regular leaf $L_{\infty} \subseteq \Delta_{\infty}$ of $\widetilde{\mathcal{F}}$ is fixed, there are real trajectories, or paths, contained in $L_{\infty}$ and possessing a contractive holonomy. To construct these trajectories we proceed as follows. The Abelian form $\omega_{1}$ induces on $L_{\infty}$ a pair of real 1-dimensional oriented singular foliations: the foliations given by $\left\{\operatorname{Im}\left(\omega_{1}\right)=0\right\}$ and by $\left\{\operatorname{Re}\left(\omega_{1}\right)=0\right\}$. Denote by $\mathcal{H}$ the oriented foliation defined by $\left\{\operatorname{Im}\left(\omega_{1}\right)=0\right\}$, the orientation being determined by the positivity of $\operatorname{Re}\left(\omega_{1}\right)$; i.e., if $\phi(t)$ is a parametrization of a leaf of $\mathcal{H}$ then $\operatorname{Re}\left(\omega_{1} \cdot \phi^{\prime}(t)\right)=\omega_{1} \cdot \phi^{\prime}(t)>0$. Each oriented trajectory of the foliation $\mathcal{H}$ will be called a real trajectory.

To make use of the foliation $\mathcal{H}$, it is clearly important to have information

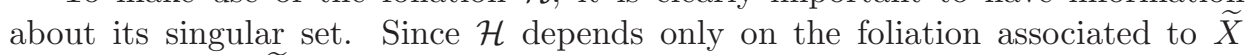
(rather than on $\widetilde{X}$ itself), we identify four "critical regions" that can give rise to singularities for $\mathcal{H}$, namely:

(1) Singular points of $\widetilde{\mathcal{F}}_{\infty}$.

(2) Points in the curve $\{H=0\}$ (assuming as before that $\operatorname{gcd}(\mathrm{P}, H)$ is a constant). 
(3) Points in the curve $\{\mathrm{P}=0\}$ (assuming as before that $\operatorname{gcd}(\mathrm{P}, H)$ is a constant).

(4) The line at infinity $\Delta_{\infty}^{(x, y)} \subset \Delta_{\infty}$ (defined by means of the affine coordinates $(x, y))$.

In the sequel we shall determine the structure of the foliation $\mathcal{H}$ in cases (2), (3) and (4) above. The discussion of singular points of $\widetilde{\mathcal{F}}_{\infty}$ will mostly be carried out in Sections 4 and 5.

Let us begin to work out the local behavior of $\mathcal{H}$ at points in the above listed "critical regions" without paying special attention to points that belong simultaneously to more than one of these regions. These latter points will be discussed in Remark 3.7 later in this section. This said, let us first consider the curve $\{H=0\}$ corresponding to zeros of $\omega_{1}$. In fact, for the time being, we shall restrict ourselves to points in the curve $\{H=0\}$ that happens to be regular for the foliation $\widetilde{\mathcal{F}}$.

Lemma 3.3. Let $p \in \Delta_{\infty}$ be a regular point of $\widetilde{\mathcal{F}}$. Assume that $p$ lies in the curve $\{H=0\} \cap \Delta_{\infty}$ (but not in $\{\mathrm{P}=0\}$ since $p$ is regular for $\left.\widetilde{\mathcal{F}}\right)$. Then $p$ is a singular point for $\mathcal{H}$. Furthermore the local structure of $\mathcal{H}$ restricted to the leaf of $\widetilde{\mathcal{F}}$ through $p$ is a saddle with $2 m$ (real) separatrices (for a certain $m \geq 1$ ).

Proof. Since $p \in \Delta_{\infty}$ is a regular point for $\widetilde{\mathcal{F}}$, it follows that $P$ does not vanish at $p$. Furthermore, at least one of the functions $F$ and $G$ does not vanish at $p$ as well. Assume, without loss of generality, that $F(p) \neq 0$. We then conclude that the restriction of $\omega_{1}$ to $L_{p}$ is holomorphic in a neighborhood of $p$ with a zero at $p$. The structure of the real foliation induced near a zero of a holomorphic 1-form on a Riemann surface is always a saddle as in the statement. Here the number $m$ of separatrices corresponds precisely to the order of $p$ as zero of $\omega_{1}$.

Let us now work out the behavior of $\mathcal{H}$ at points of $\{\mathrm{P}=0\}$ (again, only regular points for $\widetilde{\mathcal{F}}$ are considered here). Clearly it is sufficient to consider the domain of definition of the coordinates $(x, y, z)$. Similarly, if $\mathrm{P}=\mathrm{P}_{1}^{k_{1}} \cdots \mathrm{P}_{l}^{k_{l}}$ is the decomposition of $\mathrm{P}$ into irreducible components, then it suffices to consider the curve $\left\{\mathrm{P}_{1}^{k_{1}}=0\right\}$.

Lemma 3.4. Suppose that $\left\{\mathrm{P}_{1}=0\right\} \cap \Delta_{\infty}$ is not invariant by $\widetilde{\mathcal{F}}_{\infty}$. If $k_{1} \geq 2$ then $\omega_{1}$ has a pole of order $k_{1} \geq 2$ at a generic point $p$ of this curve so that $\mathcal{H}$ has a saddle-singularity at $p$. On the other hand, if $k_{1}=1$, then $\omega_{1}$ has a simple pole at a generic point $p$ of this curve, whose residue equals $H(p) / F^{*}(p)$ where $F^{*}=F / \mathrm{P}_{1}$.

Proof. Suppose that the curve $\left\{\mathrm{P}_{1}=0\right\} \cap \Delta_{\infty}$ is not invariant under $\widetilde{\mathcal{F}}_{\infty}$. Then, at generic points in $\left\{\mathrm{P}_{1}=0\right\}$, the curve in question is transverse to $\widetilde{\mathcal{F}}_{\infty}$. The point $p$ can also be chosen sufficiently generic so that $\left\{\mathrm{P}_{1}=0\right\}$ is smooth at $p$ and no other irreducible component of $\mathrm{P}$ vanishes at $p$. Under these genericity assumptions, it follows that $F^{*}(0) \neq 0$. Moreover, since we are assuming that $\operatorname{gcd}(\mathrm{P}, H)$ is invertible, we can also assume that $H(p) \neq 0$. The 1 -form $\omega_{1}$ has therefore a pole of order $k_{1}$ whose coefficient is equal to $H(p) / F^{*}(p)$. 
Now we consider points belonging to the line at infinity $\Delta_{\infty}^{(x, y)} \subset \Delta_{\infty}$. Here the reader is reminded that we chose coordinates so that $\Delta_{\infty}^{(x, y)}$ contains no singular point of the corresponding foliations.

Lemma 3.5. Points belonging to $\Delta_{\infty}^{(x, y)}$ yield source singularities for $\mathcal{H}$ provided that the coordinates $(x, y)$ are generically chosen.

Proof. As mentioned, our choice of coordinate is such that $\Delta_{\infty}^{(x, y)}$ neither contains singular points of $\widetilde{\mathcal{F}}_{\infty}$ nor is invariant under $\widetilde{\mathcal{F}}_{\infty}$. It then follows that each point $p$ in $\Delta_{\infty}^{(x, y)}$ locally belongs to a unique leaf $L_{p}$ of $\widetilde{\mathcal{F}}_{\infty}$. Thus the map that assigns to $p$ the residue at $p$ of the 1 -form $\omega_{1}$ is globally defined on $\Delta_{\infty}^{(x, y)}$. To establish the statement, it suffices to check this residue equals 1 at a generic point of $\Delta_{\infty}^{(x, y)}$. Indeed, by a continuity argument, this will imply that the residue must be real (strictly positive) at every point $p$ in $\Delta_{\infty}^{(x, y)}$ so that all these points constitute source singularities for $\mathcal{H}$. Alternatively, the reader may use the point of view discussed in Remark 3.7.

We then consider those points where $\Delta_{\infty}^{(x, y)}$ is transverse to $\widetilde{\mathcal{F}}_{\infty}$. Let $(u, v, w)$ be new local affine coordinates for $M$ where $w$ is the coordinate transverse to $\Delta_{\infty}$ and such that the line at infinity $\Delta_{\infty}^{(x, y)}$ is given by $\{u=w=0\}$. The standard associated change of coordinates is then given by $(u, v, w) \longmapsto(1 / u, v / u, w)=$ $(x, y, z)$. In these new coordinates, the vector field $\widetilde{X}$ becomes (up to multiplication by $\left.w^{1-d}\right)$

$$
-u^{2} F(1 / u, v / u) \frac{\partial}{\partial u}+u(-v F(1 / u, v / u)+G(1 / u, v / u)) \frac{\partial}{\partial v}+w H(1 / u, v / u) \frac{\partial}{\partial w} .
$$

Recall that the polynomial vector field $F(x, y) \partial / \partial x+G(x, y) \partial / \partial y$ has degree $d+1$. Furthermore its component of degree $d+1$ has the form $f(x, y)[x \partial / \partial x+y \partial / \partial y]$ where $f$ is homogeneous of degree $d$ (see Lemma 3.1). In particular, $u^{2} F(1 / u, v / u$ ) has a pole of order $d-1$ over $\{u=0\}$. Similarly the top degree homogeneous component of $-v F(1 / u, v / u)+G(1 / u, v / u)$ vanishes identically so that $\Delta_{\infty}^{(x, y)}$ represents a polar component of degree $d-1$ for the component of $\widetilde{X}$ in the $v$-direction as well. Finally, the order of poles of $H(1 / u, v / u)$ over $\Delta_{\infty}^{(x, y)}$ equals $d$. Formula (3.4) then shows that $\omega_{1}$ has poles of order 1 over $\Delta_{\infty}^{(x, y)}$. Indeed the principal part of $\omega_{1}$ is simply $1 / u$, since the top degree homogeneous component of $\widetilde{X}$ is given by $(3.2)$. The statement follows at once.

Before proceeding further, we summarize the information so far obtained about the singular set of $\mathcal{H}$ in the "critical regions" (2), (3), and (4).

(a) The regular points of $\widetilde{\mathcal{F}}_{\infty}$ contained in $\{H=0\} \cap \Delta_{\infty}$ always provide singular points for $\mathcal{H}$. Such singular points correspond to saddles with $2 m$ (real) separatrices, for $m \geq 1$.

(b) The generic points in $\{\mathrm{P}=0\} \cap \Delta_{\infty}$ also provide singular points for $\mathcal{H}$. These points can provide either poles of order $\geq 2$ for $\omega_{1}$ or poles of order 1 for $\omega_{1}$. In the first case, the corresponding singular behavior of $\mathcal{H}$ corresponds to a saddle. 
(c) The points belonging to the line at infinity always yield singular points for $\mathcal{H}$. More precisely, they provide simple poles with residue equal to 1 and therefore yield source singularities for $\mathcal{H}$.

Concerning item (b) above, in the case where $\omega_{1}$ has a simple pole at a generic point, relevant information on the residue cannot be obtained without further information on the vector field. In fact, the residue (which is given by $H(p) / F^{*}(p)$ ) takes its values in $\mathbb{C}^{*}$. Nonetheless, if the vector field $\widetilde{X}$ is supposed to be semicomplete, then the mentioned residue must belong to $\mathbb{R}^{*}$. Therefore, the singular point corresponds to a sink (resp. source) provided that the residue belongs to $\mathbb{R}_{-}$ (resp. $\mathbb{R}_{+}$). This is the content of the next lemma.

Lemma 3.6. Assume that $\left\{\mathrm{P}_{1}=0\right\} \cap \Delta_{\infty}$ is not invariant under $\widetilde{\mathcal{F}}_{\infty}$ and that $k_{1}=1$. Assume in addition that $X$ is semi-complete. Then $\omega_{1}$ has a simple pole at a generic point $p$ of the curve $\left\{\mathrm{P}_{1}=0\right\} \cap \Delta_{\infty}$ and the residue of $\omega_{1}$ at $p$ belongs to $\mathbb{R}^{*}$.

Proof. Suppose that the curve $\left\{\mathrm{P}_{1}=0\right\} \cap \Delta_{\infty}$ is not invariant by $\widetilde{\mathcal{F}}_{\infty}$. Then, at generic points of $\left\{\mathrm{P}_{1}=0\right\}$ this curve intersects $\widetilde{\mathcal{F}}_{\infty}$ transversely. Consider a generic point $p \simeq(0,0,0) \in\left\{\mathrm{P}_{1}=0\right\} \cap \Delta_{\infty}$ and local coordinates $(u, v, z)$ about $p$ where the foliation $\widetilde{\mathcal{F}}_{\infty}$ is locally represented by $\partial / \partial u$ and where $\mathrm{P}_{1}(u, v)=u$ (note that the point $p$ can be chosen so that $\left\{\mathrm{P}_{1}=0\right\}$ is smooth at $p$ ). Modulo choosing $p$ sufficiently generic to ensure that neither $H$ nor any other irreducible component of $\mathrm{P}$ vanishes at $p$, the vector field $\widetilde{X}$ takes the local form

$$
\widetilde{X}=z^{1-d} Q(u, v)\left[u f(u, v) \frac{\partial}{\partial u}+z h(u, v) \frac{\partial}{\partial z}\right]
$$

where $f(0,0) \neq 0$ and $h(0,0) \neq 0$. Now the semi-complete character of $X$ ensures that the quotient of the eigenvalues of the linear part of the vector field $u f(u, v) \partial / \partial u+z h(x, y) \partial / \partial z$ is a rational number. In other words, the quotient $h(0,0) / f(0,0)$ belongs to $\mathbb{Q}^{*} \subseteq \mathbb{R}^{*}$. This quotient, however, also represents the residue of $\omega_{1}$ at $p \simeq(0,0,0)$. The lemma is proved.

Remark 3.7. (A comment about the local behavior of $\mathcal{H}$ about certain degenerate intersection points). The purpose of this remark is to explain why certain more degenerate points belonging to the intersection of different curves as above need not be singled out in our discussion. Naturally, this discussion concerns only those points that are also regular for $\widetilde{\mathcal{F}}_{\infty}$ since the singular points of this foliation will carefully be discussed later.

First note that the intersection points of the curves $\{H=0\}$ and $\{\mathrm{P}=0\}$ are singular for the foliation since $\operatorname{gcd}(\mathrm{P}, H)$ is a constant. Also, in $\Delta_{\infty}$, all points of the curve $\{\mathrm{P}=0\}$ are singular for the foliation. Furthermore, the polynomial $\mathrm{P}$ will be constant in most of our applications.

Nonetheless points belonging to the line at infinity $\Delta_{\infty}^{(x, y)}$ need additional comment. First recall that our generic choice of generic affine coordinates $(x, y)$ is such that $\Delta_{\infty}^{(x, y)}$ neither contains singularities of $\widetilde{\mathcal{F}}_{\infty}$ nor is invariant under this 
foliation. Among points in $\Delta_{\infty}^{(x, y)}$, there are three class of non-generic points that may be regarded as more degenerate than generic points. Namely, we have intersection points with $\{H=0\}$, with $\{\mathrm{P}=0\}$ and those points where $\widetilde{\mathcal{F}}_{\infty}$ fails to be transverse to $\Delta_{\infty}^{(x, y)}$ (i.e., tangency points). The reader is reminded that, while intersection points of $\{H=0\}$ and $\{\mathrm{P}=0\}$ are singular points of $\widetilde{\mathcal{F}}$, they may be regular points for $\widetilde{\mathcal{F}}_{\infty}$. Hence, in principle, some of the intersections points of $\{H=0\}$ and $\{\mathrm{P}=0\}$ may lie in $\Delta_{\infty}^{(x, y)}$. In any event, the collection of all these points form a finite set.

We claim that the exact nature of the singularity of $\mathcal{H}$ at a point belonging to the above mentioned finite set need not be worked out. The reason for this is as follows. Consider local coordinates $(u, v, w)$ identifying the point in question with the origin of $\mathbb{C}^{3}$ and such that the foliation is locally represented by the vector field $\partial / \partial u$. In particular, the domain of definition of these local coordinates contains a single point of the finite set in question, which is identified with the origin and contained in the local leaf $L_{0}=\{v=w=0\}$. In this sense, the trajectories of $\mathcal{H}$ on the remaining leaves are well-defined, i.e., their local behavior is supposed to have been determined. Next, consider a polydisc $B(\epsilon)$ of radius $\epsilon>0$ about the origin. The behavior of $\mathcal{H}$ away from $B(\epsilon)$ is hence determined, including for those trajectories contained in the leaf $L_{0}$. The trajectories on $\mathcal{H}$ lying in $L_{0} \cap B(\epsilon)$ can then be defined through the corresponding trajectories lying in leaves different from $L_{0}$. For example, consider a point $\left(u_{0}, 0,0\right)$ lying in the boundary of $B(\epsilon)$. To define the trajectory of $\mathcal{H}$ through $\left(u_{0}, 0,0\right)$, we consider a sequence of points $\left(u_{0}, \delta_{1}, \delta_{2}\right)$ converging to $\left(u_{0}, 0,0\right)$ and the corresponding $\mathcal{H}$-trajectories $l_{\delta_{1} \delta_{2}}$ through these points. On the complement of $B(\epsilon)$, these trajectories converge to (disconnected) segments of $\mathcal{H}$-trajectories contained in $L_{0}$. We can then use as the trajectory through $\left(u_{0}, 0,0\right)$ inside $L_{0} \cap B(\epsilon)$ any segment joining two connected components of the above mentioned segments of $\mathcal{H}$-trajectories contained in $L_{0}$. In this way, the behavior of the $\mathcal{H}$-trajectories in $L_{0} \cap B(\epsilon)$ is fully determined by the behavior of $\mathcal{H}$ at the boundary of $B(\epsilon)$. For example, suppose that for every point in the boundary of $B(\epsilon)$, the corresponding $\mathcal{H}$-trajectory is oriented inward along the polydisc $B(\epsilon)$. Then the trajectories of $\mathcal{H}$ inside $L_{0} \cap B(\epsilon)$ should be regarded as exhibiting a sink singularity at the origin. In other words, we should consider that these oriented trajectories have an endpoint where they meet the boundary of $B(\epsilon)$; see Definition 3.8.

Alternatively, the reader may also consider the argument provided in the proof of Lemma 3.5 pointing out a sort of dominant behavior of $\Delta_{\infty}^{(x, y)}$ over the other critical regions.

To close this section, let us introduce the global notion of a trajectory of the foliation $\mathcal{H}$ under the condition that the trajectory in question remains away from the singular set of $\widetilde{\mathcal{F}}$. For this, it is also convenient to consider the standard Euclidean metric on the affine copy of $\mathbb{C}^{3}$ in which the affine coordinates $(x, y, z)$ are defined. In fact, the presence of an auxiliary metric will be necessary since we shall want to define also the length of a global trajectory. Here, the notion of endpoint of a trajectory will be needed; see Definition 3.8 below. We emphasize that 
all definitions provided in the sequel are only valid for (segments of) trajectories avoiding some small neighborhood of the singular set of $\widetilde{\mathcal{F}}_{\infty}$. These definitions will then be completed in Section 5 once the local behavior of $\mathcal{H}$ about these singular points will have been determined.

To motivate the definition, we first given some geometric interpretation of the foliated 1-form $\omega_{1}$. Fix a regular leaf $L_{\infty} \subseteq \Delta_{\infty}$ and a point $p_{0} \in L_{\infty}$ that is regular for $\widetilde{\mathcal{F}}$. Let $l$ be the real trajectory of $\mathcal{H}$ through $p$ and let $S$ be the cone over $L_{\infty}$. Consider a parametrization $c:[0,1] \rightarrow l$ of the segment of this trajectory joining $p_{0}=c(0)$ to $p_{1}=c(1)$. Then the holonomy map $\operatorname{Hol}(c): \Sigma_{0} \rightarrow \Sigma_{1}$, where $\Sigma_{0}$ and $\Sigma_{1}$ are vertical complex lines equipped with the coordinates $z$, satisfies

$$
\left|(\operatorname{Hol}(c))^{\prime}\right|=e^{-\operatorname{Re}\left(\int_{c} \omega_{1}\right)}<1 .
$$

Clearly this formula means that the holonomy map in question is contractive. The role played by these trajectories in our discussion can be summarized as follows. Near a sink singularity $p$ of $\mathcal{H}$, all $\mathcal{H}$-trajectories converge to $p$. The estimate (3.5) guarantees that the distance of the leaves of $\widetilde{\mathcal{F}}_{S}$ to $L_{\infty}$ has a local minimum at $p$ (which may well be zero). On the other hand, near a source $p$, all real trajectories go away from $p$. This means that the distance of the leaves of $\widetilde{\mathcal{F}}_{S}$ to $L_{\infty}$ reaches a local maximum at $p$.

We can now give a global definition of the trajectories of $\mathcal{H}$. The reader is again reminded that, until Section 5 , these definitions are only valid provided that the trajectory in question remains away from the singular set of $\widetilde{\mathcal{F}}_{\infty}$. On the other hand, recall that at regular points of $\widetilde{\mathcal{F}}_{\infty}$, the foliation $\mathcal{H}$ can have only three types of singularities, namely sources, sinks, and saddles.

Definition 3.8. A point $p$ regular for $\widetilde{\mathcal{F}}_{\infty}$ is a future endpoint (resp. past endpoint) for a trajectory of $\mathcal{H}$ if and only if $\mathcal{H}$ has a sink singularity (resp. source singularity) at $p$.

Thus, by definition, only sink or source singularities of $\mathcal{H}$ (corresponding to maxima or minima for the distance function to $\Delta_{\infty}$ restricted to the leaf) can be endpoints for a trajectory of $\mathcal{H}$. Hence, to have a global definition of $\mathcal{H}$ trajectories it only remains to say how they are defined on a neighborhood of a saddle singularity of $\mathcal{H}$. For this, recall that a saddle singularity has an even number $(2 m)$ of separatrices, $m$ of them converging to the singular point and $m$ of them emanating from the singular point. We now have:

Definition 3.9. Suppose that $l$ is a segment of trajectory of $\mathcal{H}$ converging, as a separatrix, to a saddle singularity of $\mathcal{H}$. This segment of trajectory $l$ is then continued from this singular point by following any of the local separatrices that emanate from the singular point.

The reader will not fail to observe that a trajectory of $\mathcal{H}$ passing through saddle singularities of $\mathcal{H}$ keeps giving rise to holonomy maps having a contractive behavior; this observation explains why saddle singular points are not considered as endpoints for the trajectories of $\mathcal{H}$. 
The length of an $\mathcal{H}$-trajectory is then defined by summing up its lengths (for the Euclidean metric fixed above) over foliated coordinates and this procedure is conducted simply by locally following the orientation. More precisely, consider a point $p$ and denote by $l_{p}^{+}$the (oriented) semi-trajectory of $\mathcal{H}$ starting at $p$. The length of $l_{p}^{+}$is obtained by adding lengths of its (local) segments provided that this trajectory can locally be continued (and regardless of whether or not we pass several times over the same points of $M$ ). The definition of length for an entire trajectory $l$ of $\mathcal{H}$ (as opposed to a semi-trajectory) naturally follows. As a consequence of the preceding, the length of $l_{p}^{+}$is finite if and only if $l_{p}^{+}$has a future endpoint (i.e., $l_{p}^{+}$meets a sink singularity). In particular, if $l_{p}^{+}$becomes periodic, then its length is automatically infinite.

We can now extend the previous definitions to trajectories of $\mathcal{H}$ defined on all of $M$ and not only on $\Delta_{\infty}$. In fact, the real oriented foliation $\mathcal{H}$ or, equivalently, the 1 -form $\omega_{1}$ has been introduced for leaves contained in $\Delta_{\infty}$. As soon as a leaf $L_{\infty} \subseteq \Delta_{\infty}$ is fixed, the definition can naturally be adapted to every leaf on $S$. Going back to our specific case in which $\omega_{1}$ is characterized by the formula (3.4), it follows that the local trajectories of $\mathcal{H}$ on $L \subseteq S$ are determined as the lifts in $T_{(x, y(x))} L_{\infty}$ of the vector $v$ where $v$ is such that $v \cdot H(x, y(x)) / F(x, y(x))$ belongs to $\mathbb{R}_{-}$. Also, note that the corresponding Abelian form $\omega_{1}$ is independent of the leaf in the same cone $S$. In fact, the equation (3.4) shows that it depends solely on $L_{\infty}$. These remarks can be summarized as follows.

1. The trajectory of $\mathcal{H}$ through a point $\left(p_{1}, p_{2}, p_{3}\right)$ projects on the trajectory of $\mathcal{H}$ through the point $\left(p_{1}, p_{2}, 0\right)$ which, in addition, is globally contained in the plane $\{z=0\}$.

2. Since the absolute value of the coordinate " $z$ " is always decreasing over a trajectory of $\mathcal{H}$, it follows that the trajectory of $\mathcal{H}$ through $\left(p_{1}, p_{2}, p_{3}\right)$ has infinite length if and only if the the trajectory of $\mathcal{H}$ through $\left(p_{1}, p_{2}, 0\right)$ has infinite length.

The following simple lemma will also be important later. For this lemma we should take into account that, whereas $\Delta_{\infty}^{(x, y)}$ can be chosen to be generic, it always possesses points of tangency with the foliation $\widetilde{\mathcal{F}}_{\infty}$.

Lemma 3.10. For a generic choice of the affine coordinates $(x, y)$ an oriented trajectory of $\mathcal{H}$ never intersects $\Delta_{\infty}^{(x, y)}$. Moreover there is a compact set $K \subset \mathbb{C}^{3}$ and a constant $C_{K}$ so that the following holds: every segment of a trajectory $l$ of $\mathcal{H}$ whose total length is greater than $C_{K}$ satisfies the condition that the part of l lying in $\mathbb{C}^{3} \backslash K$ is less than, say, 1/10 of the total length of the segment in question.

Proof. Let $q_{1}, \ldots, q_{r}$ be the points where $\Delta_{\infty}^{(x, y)}$ is tangent to $\widetilde{\mathcal{F}}_{\infty}$ and fix a small neighborhood $W_{i}$ of $q_{i}, i=1, \ldots, r$. Then there is a (collar) neighborhood $V$ of $\Delta_{\infty}^{(x, y)} \backslash \bigcup_{i=1}^{r} W_{i}$ so that the following holds: for every point $p \in \partial V \backslash \bigcup_{i=1}^{r} W_{i}$ the trajectory of $\mathcal{H}$ through $p$ is transverse to $\partial V$ and oriented outwards along $V$. In other words, no trajectory of $\mathcal{H}$ may enter $V$ without first entering some $W_{i}$.

On the other hand the structure of $\mathcal{H}$ trajectories on $W_{i}$ is easy to describe. If $\Delta_{\infty}^{(x, y)}$ is generic, then the tangency of $\Delta_{\infty}^{(x, y)}$ at $\widetilde{\mathcal{F}}_{\infty}$ at $q_{i}$ is quadratic (for all 
$i \in\{1, \ldots, r\})$. In particular if $L_{i}$ is the local leaf of $\widetilde{\mathcal{F}}_{\infty}$ through $q_{i}$, the point $q_{i}$ is itself a source singularity for the trajectories of $\mathcal{H}$. Thus no trajectory of $\mathcal{H}$ intersects $\Delta_{\infty}^{(x, y)}$. Finally, if $V$ and the neighborhoods $W_{i}$ are sufficiently small, then the length of a segment of a trajectory lying in $V \cup \bigcup_{i=1}^{r} W_{i}$ is less than, say, $1 / 30$ the length of the segment of same trajectory in $K=\mathbb{C}^{3} \backslash V \cup \bigcup_{i=1}^{r} W_{i}$ which is determined by two successive passes of the trajectory in question through $V \cup \bigcup_{i=1}^{r} W_{i}$. The statement then follows.

Remark 3.11. In certain cases it may be useful to make a nongeneric choice of affine coordinates $(x, y)$ so as to have a line at infinity $\Delta_{\infty}^{(x, y)}$ passing through certain singular points of $\widetilde{\mathcal{F}}_{\infty}$. We shall briefly mention one situation of this type later on; see Remark 6.8.

\section{Renormalization in the exceptional divisor}

Our fundamental tool to derive Theorem $\mathrm{A}$ is a procedure of renormalization for the complex time near the divisor of poles of $\widetilde{X}$, i.e., near $\Delta_{\infty}$. This construction will play a major role in the rest of the paper. We begin by describing this procedure. We shall continue to use the notation of Section 3, emphasizing the 3-dimensional case, though all the results presented below are valid in arbitrary dimensions.

As before, let $X$ stand for a homogeneous polynomial vector field of degree $d \geq 2$ and assume that $X$ is not a multiple of the radial vector field. Denote by $\mathcal{F}$ the foliation associated with $X$. The reader is also reminded that leaves for the foliation $\mathcal{F}$ are defined as in Section 2.2. In particular, the restriction of $X$ to a leaf $L$ of $\mathcal{F}$ may contain zeros, poles, and essential singularities of $X$. All these nonregular points of $X$ form, however, a discrete subset of $L$ with respect to the intrinsic topology of the leaf $L$ as Riemann surface (unless the leaf in question is fully contained in the divisor of zeros and poles of $X$ ). The presence of the vector field $X$ allows us to endow every (regular) leaf $L$ as before with the foliated timeform $d T$ defined, as in Section 2.2, by imposing $d T . X=1$. The time-form will also be denoted by $d T_{L}$ when we want to emphasize the leaf $L$ under consideration. As observed, the time-form is well-defined provided that $L$ is not contained in the divisor of zeros and poles of $X$. If the vector field $X$ is supposed to be semicomplete, then its restriction to $L$ is everywhere holomorphic and the orders of its zeros cannot exceed 2; see Lemma 2.4. It follows at once that $d T$ is meromorphic on all of $L$ and it has no zeros. Furthermore, the poles of $d T$ have order bounded by 2 . Finally, recall also that given a curve $c:[0,1] \rightarrow L$ joining two points $c(0)$ and $c(1)$ in $L$ satisfying $X(c(0)) \neq 0$ and $X(c(1)) \neq 0$, the integral $\int_{c} d T$ measures the time needed to traverse $c$ from $c(0)$ to $c(1)$ following the flow of $X$ as long as $X$ is semi-complete. In fact, when a vector field is semi-complete the notion of time arising from its semi-global flow is well-defined.

Consider now $\widetilde{X}$, the vector field induced by $X$ on $M$. Throughout this section, generic affine coordinates $(x, y, z)$ as in Section 3 are fixed. In particular, $\Delta_{\infty}^{(x, y)}$ neither contains singular points of $\widetilde{\mathcal{F}}_{\infty}$ nor is invariant under this foliation. Since $\tilde{X}$ 
has poles over $\Delta_{\infty}$, the time-form is not defined for the regular leaves of $\widetilde{\mathcal{F}}_{\infty}$. It is, however, possible to define a renormalized time-form on a neighborhood of each regular point $p$ of a leaf $L_{\infty} \subseteq \Delta_{\infty}$. This goes as follows. Let $L_{\infty} \subseteq \Delta_{\infty}$ be a regular leaf of $\widetilde{\mathcal{F}}$ and let $p \in \bar{L}_{\infty}$ be a regular point of this leaf $L_{\infty}$ which is not singular for $\widetilde{\mathcal{F}}$. Choose local coordinates $(u, v, w),\{w=0\} \subset \Delta_{\infty}$ around $p$ where the foliation is given by the vector field $\partial / \partial u$. In these coordinates, the vector field $\widetilde{X}$ is given by $w^{1-d} f(u, v, w) \partial / \partial u$. The renormalized time-form on $L_{\infty}$ is then defined as $d u / f(u, 0,0)$. In other words, it is obtained from $\tilde{X}$ by eliminating its pole component. Naturally there is no canonical choice for the coordinate $w$ and this prevents us from having a global definition for the renormalized timeform. In accurate terms, the local form $d u / f(u, 0,0)$ is not globally defined on $L_{\infty}$ because, when a change of coordinates is performed, two local definitions of this renormalized time-form will agree only up to a multiplicative constant. Therefore, whereas the previous construction does not define an Abelian form on $L_{\infty}$, it endows $L_{\infty}$ with an affine structure (for further details we refer to [29]). The purpose of this section is to exploit this affine structure to estimate the domain of definition of the solutions of $\widetilde{X}$. As will be seen, accurate estimates can be obtained in this way as long as the evolution of the coordinate $z$ is well controlled (where $z$ refers to the affine coordinates $(x, y, z)$ ).

Although we have defined the renormalized time-form only at regular points of $\widetilde{\mathcal{F}}$, this form admits a natural asymptotic extension to the singularities of $\widetilde{\mathcal{F}}$ lying in $\Delta_{\infty}$. Details on these extensions will be given as they become necessary.

Now let us return to homogeneous polynomial vector fields on $\mathbb{C}^{3}$. Fix a point $p_{0}$ contained in the singular set of $\widetilde{\mathcal{F}}_{\infty}$. Suppose that the restriction of $\widetilde{\mathcal{F}}$ to a neighborhood of $p_{0}$ is given by the equation (3.1) so that

$$
\widetilde{X}=\frac{1}{z^{d-1}}\left[F(x, y) \frac{\partial}{\partial x}+G(x, y) \frac{\partial}{\partial y}+z H(x, y) \frac{\partial}{\partial z}\right] .
$$

With the notations of Section 3 , let $\mathrm{P}=\operatorname{gcd}(F, G)$ so that $F=\mathrm{P} . a(x, y)$ and $G=\mathrm{P} . b(x, y)$. Denoting by $\overline{\mathrm{P}}$ the greatest common divisor of $\mathrm{P}$ and $H$, we can set $\mathrm{P}=\overline{\mathrm{P}} \mathrm{P}^{*}$ and $H=\overline{\mathrm{P}} H^{*}$. It follows that

$$
\widetilde{X}=\frac{\overline{\mathrm{P}}}{z^{d-1}}\left[\mathrm{P}^{*}(x, y)\left(a(x, y) \frac{\partial}{\partial x}+b(x, y) \frac{\partial}{\partial y}\right)+z H^{*}(x, y) \frac{\partial}{\partial z}\right]
$$

where $p_{0} \simeq(0,0,0)$. If $\mathrm{P}^{*}$ is not constant, the curve in $\Delta_{\infty}$ induced by $\left\{\mathrm{P}^{*}=0\right\}$ is singular for $\widetilde{\mathcal{F}}$, though its generic points are regular for $\widetilde{\mathcal{F}}_{\infty}$. From this point of view $\left\{\mathrm{P}^{*}=0\right\} \cap \Delta_{\infty}$ may or may not be invariant under $\widetilde{\mathcal{F}}_{\infty}$. Nonetheless, when dealing with semi-complete vector fields, the following holds.

Proposition 4.1. Assume that $X$ is a homogeneous semi-complete vector field with degree greater than or equal to 3. Suppose that $\widetilde{X}$ is as in (4.2). Then no irreducible component of $\left\{\mathrm{P}^{*}=0\right\} \cap \Delta_{\infty}$ is invariant under $\widetilde{\mathcal{F}}_{\infty}$. In other words, a regular leaf of $\widetilde{\mathcal{F}}_{\infty}$ can intersect the singular set of $\widetilde{\mathcal{F}}$ only in a discrete set (for the intrinsic topology on the leaf in question). 
Proof. Without loss of generality, denote by $\mathrm{P}_{1}$ an irreducible component of $\mathrm{P}^{*}$ giving rise to an (irreducible) curve $\mathcal{C}=\left\{\mathrm{P}_{1}=0\right\} \cap \Delta_{\infty}$ that happens to be invariant under $\widetilde{\mathcal{F}}_{\infty}$. We are going to conclude from this condition that $X$ cannot be semi-complete.

To do this, denote by $m \geq 1$ the multiplicity of $\mathrm{P}_{1}$ as a component of $\mathrm{P}^{*}$. At a generic point of $\mathcal{C}$, local coordinates $(u, v, w)$ can be found so that $\{w=0\} \subset \Delta_{\infty}$ and $\mathcal{C}$ is identified with $\{v=0, w=0\}$. In these coordinates, we naturally have $\mathrm{P}_{1}(u, v)=v$. Moreover, since the chosen point is generic, we also have $H(0,0) \neq 0$ and $a(0,0) \neq 0$. On the other hand, $b$ must be divisible by $v$ since $\mathcal{C}$ is invariant under $\widetilde{\mathcal{F}}_{\infty}$.

By means of the above defined local coordinates, $\widetilde{X}$ can be identified with a vector field defined around the origin of $\mathbb{C}^{3}$. Since $m \geq 1$, the first nonzero homogeneous component of $\widetilde{X}$ at the origin is given by

$$
\tilde{X}^{H}=w^{1-d} v^{k}\left[\alpha v \frac{\partial}{\partial u}+\lambda w \frac{\partial}{\partial w}\right]
$$

for some constants $\lambda=H(0,0) \in \mathbb{C}^{*}, k \geq 0$ and $\alpha \in \mathbb{C}$. Note that $\alpha \neq 0$ if and only if $m=1$. Furthermore, $k$ is the greatest (nonnegative) integer such that $v^{k}$ divides $\overline{\mathrm{P}}$. The hyperplanes $\{v=$ cte $\}$ are invariant under the foliation associated with $\widetilde{X}^{H}$. For each sufficiently small nonzero constant (cte), the differential equation associated with $\widetilde{X}^{H}$ is such that $\dot{w}=\operatorname{cte}^{k} \lambda w^{2-d}$. Since $d \geq 3$, the vector field $\widetilde{X}^{H}$ has a pole at $w=0$. In turn, the existence of this pole ensures that the corresponding solution is multivalued contradicting the assumption that $X$ is semi-complete. The proof of the lemma is finished.

Concerning the case of quadratic homogeneous vector fields, the preceding lemma can be complemented nicely under the additional assumption that the singular set of $X$ has codimension greater than or equal to 2. Namely, we have:

Proposition 4.2. Assume that $X$ is a quadratic homogeneous semi-complete vector field whose singular set has codimension greater than or equal to 2 . Suppose that $\widetilde{X}$ is as in (4.2). Then no irreducible component of $\left\{\mathrm{P}^{*}=0\right\} \cap \Delta_{\infty}$ is invariant under $\widetilde{\mathcal{F}}_{\infty}$.

Proof. Let $\mathrm{P}_{1}$ denote a nontrivial irreducible component of $\mathrm{P}^{*}$ and assume, aiming at a contradiction, that $\left\{\mathrm{P}_{1}=0\right\} \cap \Delta_{\infty}$ is invariant under $\widetilde{\mathcal{F}}_{\infty}$. Denote by $L$ the intersection $\left\{\mathrm{P}_{1}=0\right\} \cap \Delta_{\infty}$ and note that $L$ is contained in a leaf of $\widetilde{\mathcal{F}}_{\infty}$. Fix $p \in L$ such that $\widetilde{\mathcal{F}}_{\infty}$ is regular at $p$. The point $p$ can be chosen so that neither $H$ nor any other irreducible component of $\mathrm{P}^{*}$ vanishes at $p$. Next, consider local coordinates $(u, v, w)$ about $p,\{w=0\} \subset \Delta_{\infty}$, so that

(a) $p \simeq(0,0,0)$;

(b) $\mathrm{P}(u, v)=v$;

(c) the foliation $\widetilde{\mathcal{F}}_{\infty}$ is horizontal, i.e., is represented by the vector field $\partial / \partial u$. 
Denote by $\alpha$ the order of $\mathrm{P}_{1}$ as a component of $\mathrm{P}^{*}$ and note that $\alpha$ cannot exceed 3 since $X$ has degree 2 . In these coordinates the vector field $\widetilde{X}$ becomes

$$
\tilde{X}=\frac{1}{w}\left[v^{\alpha} f(u, v) \frac{\partial}{\partial u}+w g(u, v) \frac{\partial}{\partial w}\right] .
$$

Furthermore, modulo taking $p$ sufficiently generic, it can also be assumed that both $f(0,0)$ and $g(0,0)$ are different from zero.

Let $\widetilde{\mathcal{F}}$ denote the foliation associated with $\widetilde{X}$. To complete the proof of the proposition, we are going to show that the local holonomy of $\widetilde{\mathcal{F}}$ with respect to the invariant axis $\{u=v=0\}$ does not coincide with the identity map. This contradicts the assumption that $\tilde{X}$ is semi-complete since the restriction of $\tilde{X}$ to the mentioned axis is the regular (constant) vector field $g(0,0) \partial / \partial x$ (the reader is reminded that $g(0,0) \neq 0)$. In fact, being regular, the integral of the time-form over a loop encircling the origin is zero. If the local holonomy of the mentioned invariant axis is not trivial, then this loop lifts to an open path in a nearby leaf $L$. Since the intrinsic distance in $L$ between the endpoints of this open path is bounded below by a positive constant, there follows easily the existence of an open path $c \subset L$ over which the integral of the corresponding time-form $d T_{L}$ vanishes which is impossible for a semi-complete vector field; see Section 2.2.

To compute the local holonomy map associated to $\{u=v=0\}$ with respect to the foliation $\widetilde{\mathcal{F}}$, consider the loop given by $w(t)=e^{2 \pi i t}$. Set $h(u, v)=$ $f(u, v) / g(u, v)$ and note that $h$ is holomorphic about $(0,0)$ with $h(0,0) \neq 0$ since both $f(0,0)$ and $g(0,0)$ are assumed to be nonzero. Now, there follows that $(u(t), v(t))$ satisfies the differential equation

$$
\left\{\begin{array}{l}
\frac{d u}{d t}=\frac{d u}{d w} \frac{d w}{d t}=2 \pi i v^{\alpha} h(u, v), \\
\frac{d v}{d t}=\frac{d v}{d w} \frac{d w}{d t}=0 .
\end{array}\right.
$$

Next, by setting

$$
u(t)=\sum_{j, k} a_{j k}(t) u_{0}^{j} v_{0}^{k} \quad \text { and } \quad v(t)=\sum_{j, k} b_{j k}(t) u_{0}^{j} v_{0}^{k},
$$

the equation $d v / d t=0$ implies that $b_{01}(t)$ is constant equal to 1 and that $b_{j k}(t)$ vanishes identically for every pair $(j, k) \neq(0,1)$. This is equivalent to saying that $v(t)=v_{0}$ for all $t$.

Consider now the second equation. Let $h(u, v)=\sum_{n, m} h_{n m} u^{n} v^{m}$. From the equation $d u / d t=2 \pi i v^{\alpha} h(u, v)$, we conclude that

$$
\sum_{j, k} a_{j k}^{\prime}(t) u_{0}^{j} v_{0}^{k}=2 \pi i v_{0}^{\alpha} \sum_{n, m} h_{n m}\left(\sum a_{j k}(t) u_{0}^{j} v_{0}^{k}\right)^{n} y_{0}^{m}
$$

which, in turn, is equivalent to

$$
\sum_{j, k} a_{j k}^{\prime}(t) u_{0}^{j} v_{0}^{k}=\sum_{n, m} 2 \pi i h_{n m}\left(\sum_{j, k} a_{j k}(t) u_{0}^{j} v_{0}^{k}\right)^{n} v_{0}^{m+\alpha} .
$$


Comparing the coefficient of the monomial $v_{0}^{\alpha}$ in both the right and left sides of the preceding equation, it follows that

$$
a_{0 \alpha}^{\prime}(t)=2 \pi i h_{00},
$$

where $h_{00}=h(0,0) \neq 0$. Since $a_{0 \alpha}(0)$ equals zero, we obtain that $a_{0 \alpha}(t)=2 \pi i h_{00} t$ and, therefore,

$$
a_{0 \alpha}(1)=2 \pi i h_{00} \neq 0 \text {. }
$$

Hence the holonomy map $\left(u_{0}, v_{0}\right) \mapsto(u(1), v(1))$ does not coincide with the identity since $u(1)$ is not independent of $v_{0}$. This yields the desired contradiction and ends the proof of Proposition 4.2.

Unlike the time-form, the renormalized time-form is defined for every regular leaf of the foliation whether or not the leaf is contained in the zero/pole divisor of $X$. Propositions 4.1 and 4.2 then imply that the "renormalized time-form" can be defined over every leaf $L_{\infty} \subseteq \Delta_{\infty}$ provided that $X$ satisfies the conditions in the preceding statements. In view of this, and unless otherwise stated, throughout the rest of this paper we shall assume the following.

General assumption: No irreducible component of the curve $\left\{\mathrm{P}^{*}=0\right\} \cap \Delta_{\infty}$ is invariant under $\widetilde{\mathcal{F}}_{\infty}$.

As pointed out above, under the semi-completeness assumption, $\left\{\mathrm{P}^{*}=0\right\} \cap \Delta_{\infty}$ is not invariant for the foliation induced by the vector field $X$. Nonetheless, for semi-complete vector fields, a lot more can be said about this (non- $\widetilde{\mathcal{F}}_{\infty}$-invariant) curve. In particular, when $X$ is a homogeneous semi-complete vector field with degree at least 3 , the proof of Proposition 4.1 also yields:

Proposition 4.3. Assume that $X$ is a homogeneous polynomial semi-complete vector field with degree $d \geq 3$. Let $\widetilde{X}$ be as in (4.2) and assume also that $\mathrm{P}^{*}$ is not invertible. Then every nontrivial irreducible component of $\mathrm{P}^{*}$ has order 1 . Furthermore, every nontrivial irreducible component of $\mathrm{P}^{*}$ must also appear as an irreducible component of $\overline{\mathrm{P}}$.

Proof. Assume that $\mathrm{P}^{*}$ is not invertible. Let $\mathrm{P}_{1}$ be a nontrivial irreducible component of $\mathrm{P}^{*}$ and denote by $m \geq 1$ the order of $\mathrm{P}_{1}$ with respect to $\mathrm{P}^{*}$. Since $\left\{\mathrm{P}_{1}=0\right\} \cap \Delta_{\infty}$ is not invariant under $\widetilde{\mathcal{F}}_{\infty}$, at a generic point of $\left\{\mathrm{P}_{1}=0\right\} \cap \Delta_{\infty}$ this curve is transverse to $\widetilde{\mathcal{F}}_{\infty}$. A generic point $p$ can be chosen so that, in addition, neither $H$ nor any other irreducible component of $\mathrm{P}^{*}$ vanishes at $p$. Finally, we can also suppose that $\left\{\mathrm{P}_{1}=0\right\}$ is smooth at $p$. Next, consider local coordinates $(u, v, w)$ around $p,\{w=0\} \subset \Delta_{\infty}$, satisfying the following conditions:

- $p \simeq(0,0,0)$;

- $\mathrm{P}_{1}(u, v)=v$;

- The foliation $\widetilde{\mathcal{F}}_{\infty}$ is locally represented by the vector field $\partial / \partial v$. 
In other words, we have chosen coordinates $(u, v, w)$ where $\widetilde{X}$ takes the form

$$
\widetilde{X}=w^{1-d} \overline{\mathrm{P}}(u, v)\left[v^{m} g(u, v) \frac{\partial}{\partial v}+w h(u, v) \frac{\partial}{\partial w}\right],
$$

where both $g(0,0)$ and $h(0,0)$ are different from zero.

Suppose that $m>1$. Then, the first nonzero homogeneous component of $\widetilde{X}$ at the origin (identified with $p$ ) is given by

$$
\widetilde{X}^{H}=\lambda v^{k} w^{2-d} \frac{\partial}{\partial w}
$$

where $\lambda=H(0,0)$ and $k$ is the order of $\mathrm{P}_{1}(u, v)=v$ with respect to $\overline{\mathrm{P}}$. Since $d \geq 3$, the restriction of $\widetilde{X}^{H}$ to the invariant planes $\{v=$ cte $\}$ is not semi-complete provided that cte is different from zero. This contradicts the assumption that $X$ is semi-complete; see Section 2.2. It then follows that $m=1$.

From now on, we have $m=1$. It remains to prove that $k$ must be strictly positive. Thus, let us suppose for the sake of a contradiction that $k=0$. The first nonzero homogeneous component of $\widetilde{X}$ at the origin (identified with $p$ ) is hence given by

$$
\widetilde{X}^{H}=w^{1-d}\left[\alpha v \frac{\partial}{\partial v}+\lambda w \frac{\partial}{\partial w}\right]
$$

for some constant $\alpha \in \mathbb{C}^{*}$ and where $\lambda$ equals $H(0,0)$. Now, the restriction of the foliation associated with $\widetilde{X}^{H}$ to the invariant hyperplane $\{v=0\}$ is given by $w^{2-d} \partial / \partial z$ which is not semi-complete provided that $d \geq 3$; see Section 2.2. The proposition is proved.

As an immediate consequence, we have the following:

Corollary 4.4. Assume that $X$ is a homogeneous polynomial semi-complete vector field with degree at least 3 . Suppose also that the singular set of $X$ has codimension at least 2 . Then $\mathrm{P}^{*}$ is invertible, i.e., $\mathrm{P}^{*}$ is a constant.

Proof. Assume that $\mathrm{P}^{*}$ is not invertible and consider a nontrivial irreducible component $\mathrm{P}_{1}$ of $\mathrm{P}^{*}$. According to Proposition $4.3, \mathrm{P}_{1}$ must also be an irreducible component of $\overline{\mathrm{P}}$. This immediately implies that the singular set of $X$ has codimension 1 and the statement follows.

Concerning the case of homogeneous polynomial vector fields of degree $d=2$, the following holds:

Proposition 4.5. Suppose that $X$ is a homogeneous quadratic semi-complete vector field. Suppose that $\mathrm{P}^{*}$ is not invertible. Then every nontrivial irreducible component of $\mathrm{P}^{*}$ has order 1 .

Proof. Let $\mathrm{P}_{1}$ be a nontrivial irreducible component of $\mathrm{P}^{*}$ and denote by $m$ the order of $\mathrm{P}_{1}$ with respect to $\mathrm{P}^{*}$. Owing to the general assumption, the algebraic curve $\left\{\mathrm{P}_{1}=0\right\} \cap \Delta_{\infty}$ is not invariant under $\widetilde{\mathcal{F}}_{\infty}$ (cf. Proposition 4.2 ). This means 
that this algebraic curve is transverse to $\widetilde{\mathcal{F}}_{\infty}$ at a generic point of it. Moreover, on a neighborhood of a sufficiently generic point of $\left\{\mathrm{P}_{1}=0\right\} \cap \Delta_{\infty}$, there are local coordinates $(u, v, w)$ where $\widetilde{X}$ becomes

$$
\widetilde{X}=w^{1-d} \overline{\mathrm{P}}(u, v)\left[v^{m} g(u, v) \frac{\partial}{\partial v}+w h(u, v) \frac{\partial}{\partial w}\right]
$$

with both $g(0,0)$ and $h(0,0)$ different from zero. In particular, for fixed $u$, the 2dimensional vector field $v^{m} g(u, v) \partial / \partial v+w h(u, v) \partial / \partial w$ has exactly one eigenvalue different from zero at the origin provided that $m \geq 2$. By means of an elementary and well-known calculation, this fact ensures that the local holonomy arising from the axis $\{v=0\}$ cannot coincide with the identity. Therefore the corresponding vector field cannot be semi-complete since its restriction to the mentioned axis is regular at $\{v=w=0\}$ (the details are as in the proof of Proposition 4.2).

Unlike the case of homogeneous polynomial semi-complete vector fields with degree $\geq 3$, the set of homogeneous polynomial semi-complete vector fields of degree 2 admitting an irreducible component (not invariant under $\mathcal{F}$ and) contained in its singular set is not empty, even when the singular set of $X$ is supposed to have codimension at least 2 . Indeed, the homogenous vector field

$$
X=x z \frac{\partial}{\partial x}+\left(2 y z+y^{2}\right) \frac{\partial}{\partial y}+z^{2} \frac{\partial}{\partial z}
$$

is a semi-complete vector field and induces a foliation on the hyperplane at infinity admitting a noninvariant curve contained in the singular set of the foliation associated to $X$. In fact, in the standard affine coordinates $(x, y, z)$ of Section $3, \widetilde{X}$ is given by

$$
\widetilde{X}=\frac{1}{z}\left[y(1+y) \frac{\partial}{\partial y}-z \frac{\partial}{\partial z}\right] .
$$

Although the set of homogeneous polynomial semi-complete vector fields of degree $d=2$ admitting a nontrivial irreducible component (not invariant under $\mathcal{F}$ and) contained in the singular set of $\mathcal{F}$ is not empty, the corresponding vector fields will be excluded from our discussion. In fact, as far as the main results presented in the introduction are concerned, whenever the singular set of a foliation plays a specific role, this singular set is assumed to consist only of simple singularities (in the sense described in the introduction). However, simple singularities in this sense are not compatible with the presence of curves of singular points contained in the hyperplane of infinity for the foliation in question. Alternatively, it should be noted that the foliations associated to the above nonempty set of homogeneous polynomial vector fields can easily be described. In fact, we have:

Lemma 4.6. Let $X$ be a homogeneous quadratic vector field and let $\mathrm{P}^{*}$ be as above. If $\mathrm{P}^{*}$ is not invertible, then the foliation $\widetilde{\mathcal{F}}_{\infty}$ is induced by a vector field of degree 0 or 1 .

After Lemma 4.6, the case where $d=2$ and $\mathrm{P}^{*}$ is not constant can be treated directly and the details can be left to the reader. 
After this long detour, we return to the real 1-dimensional foliation $\mathcal{H}$. The rest of this section is devoted to establishing Theorem 4.7 below which, in fact, makes no assumption as to whether or not the corresponding vector field $X$ is semi-complete. This theorem will be extended further in the next section and the final result, when combined with the preceding material about semi-complete vector fields, will provide us with the required quantitative information to prove the main results stated in the introduction.

Consider then a homogeneous polynomial vector field $X$ of degree $d \geq 2$ that is not a multiple of the radial vector field. Whether or not $X$ is semi-complete, we can consider the vector field $\widetilde{X}$ on $M$ along with its associated foliation $\widetilde{\mathcal{F}}$ and the induced foliation $\widetilde{\mathcal{F}}_{\infty}$ on $\Delta_{\infty}$. Next, fix a regular leaf $L_{\infty} \subseteq \Delta_{\infty}$ of $\widetilde{\mathcal{F}}$ and let $S=\mathcal{P}_{\infty}^{-1}\left(L_{\infty}\right)$ be the cone over $L_{\infty}$. Denote by $\mathcal{H}$ the oriented 1-dimensional real foliation induced by the Abelian form $\omega_{1}$ (see Section 3). It is also useful to consider other foliations similar to $\mathcal{H}$. For this let us consider an angle $\theta \in(-\pi / 2, \pi / 2)$. Denote by $\mathcal{H}^{\theta}$ the oriented foliation whose (oriented) trajectories make an angle $\theta$ with the (oriented) trajectories of $\mathcal{H}$. It is clear that these foliations are well defined under the same conditions as $\mathcal{H}$. It is also clear that the holonomy maps of $\widetilde{\mathcal{F}}_{S}$ along the trajectories of $\mathcal{H}^{\theta}$ are still contractions as in (3.5) (up to multiplicative constants). In the sequel we denote by $l^{\theta}$ an oriented trajectory of $\mathcal{H}^{\theta}$.

Given (a segment of) a trajectory $l_{p}$ of $\mathcal{H}$ (resp. $l_{p}^{\theta}$ of $\mathcal{H}^{\theta}$ ), we are interested in the value of the integral $\int_{l_{p}} d T$ (resp. $\int_{l_{p}^{\theta}} d T$ ). In the investigation of the behavior of this integral, it is clear that the singularities of $\widetilde{\mathcal{F}}$ on $\Delta_{\infty}$ will pose further difficulties. Thus it is natural to begin with (segments of) trajectories of $\mathcal{H}$ (resp. $\mathcal{H}^{\theta}$ ) that avoid a fixed neighborhood of the corresponding singular set. In order to do this, let $W$ be a sufficiently small open neighborhood of the singular set of $\widetilde{\mathcal{F}}$ on $\Delta_{\infty}$. Let $l_{p}$ (resp. $l_{p}^{\theta}$ ) be (a segment of) a trajectory of $\mathcal{H}\left(\right.$ resp. $\mathcal{H}^{\theta}$ ). We can now state one of our main results. Despite our 3 -dimensional setting, the reader can immediately check that this result holds in arbitrary dimensions (as is always the case in the present section).

Theorem 4.7. Suppose that $l_{p}\left(\right.$ resp. $\left.l_{p}^{\theta}\right)$ is contained in $\Delta_{\infty} \backslash W$. Then $\int_{l_{q}} d T$ (resp. $\left.\int_{l_{q}^{\theta}} d T\right)$ converges for all $q=\left(p, z_{0}\right) \in \mathcal{P}_{\infty}^{-1}(p, 0)$, where $l_{q}$ (resp. $\left.l_{q}^{\theta}\right)$ denotes the lift of $l_{p}$ (resp. $\left.l_{p}^{\theta}\right)$ to the leaf of $\widetilde{\mathcal{F}}$ through $q$ and where dT stands for the time-form associated to $\widetilde{X}$. More precisely, assuming $W$ fixed, and assuming that the trajectory $l_{q}^{\theta}$ of $\mathcal{H}^{\theta}$ does not intersect $W$, there exists a constant $C$ (depending continuously on $\theta$ ) such that for every path $c:[0,1] \rightarrow L, c(0)=q$, with image contained in $l_{q}^{\theta}$, we have

$$
\left|\int_{c} d T\right| \leq \int_{c}|d T| \leq \int_{l_{q}^{\theta}}|d T|<C\left|z_{0}\right|^{d-1},
$$

where $d \geq 2$ stands for the degree of the initial homogeneous vector field $X$.

Proof. It suffices to prove the statement for the case of a trajectory $l_{p}$ of $\mathcal{H}$ since the adaptations needed for trajectories of $\mathcal{H}^{\theta}$ are clear. Also, we can suppose without loss of generality that the length of $l_{p}$ is infinite. Finally, we recall that the affine 
coordinates $(x, y)$ are as in Section 3 , namely $\Delta_{\infty}^{(x, y)}$ is not invariant under $\widetilde{\mathcal{F}}_{\infty}$ and $\Delta_{\infty}^{(x, y)}$ contains no singularities of $\widetilde{\mathcal{F}}_{\infty}$.

Let $W$ be the previously chosen open neighborhood of the intersection of $\Delta_{\infty}$ with the singular set of $\widetilde{\mathcal{F}}$. Assume that $l_{p}$ is connected and contained in $\Delta_{\infty} \backslash W$. Since the intersection of $\Delta_{\infty} \backslash W$ with the singular set of $\widetilde{\mathcal{F}}$ is empty and the length of $l_{p}$ is infinite, the only singularities of $\mathcal{H}$ that may be met by the trajectory $l_{p}$ are saddle singularities of $\mathcal{H}$ (occurring at regular points of $\widetilde{\mathcal{F}}$ ). However, according to Definition 3.9 , the corresponding trajectories of $\mathcal{H}$ are continued by following separatrices that emanate from the saddle in question. Moreover, with this global definition of $\mathcal{H}$-trajectory, the uniform contractive character of the corresponding holonomy maps is still valid.

Recall from Section 3 that, away from $W$, the polar divisor of $\omega_{1}$ consists of the line at infinity $\Delta_{\infty}^{(x, y)} \subset \Delta_{\infty}$. Moreover, the singularities of $\mathcal{H}$ at points in $\Delta_{\infty}^{(x, y)}$ are source-like so that an oriented trajectory of $\mathcal{H}$ cannot intersect $\Delta_{\infty}^{(x, y)}$. Though these trajectories of $\mathcal{H}$ may come close to $\Delta_{\infty}^{(x, y)}$, owing to Lemma 3.10 we know that every sufficiently long segment of $l_{p}$ has most of its length contained in a fixed compact subset of the affine $\mathbb{C}^{2}$ associated with the coordinates $(x, y)$. Let then a compact set $K \subset \mathbb{C}^{2}$ possessing the mentioned property be fixed. Since $F$ is clearly bounded on $K$, the estimates of Lemma 3.10 allow us to conclude that every sufficiently long segment $c_{p}$ of $l_{p}$ can be split into a concatenation $c_{1} * c_{2} * \cdots * c_{k}$ such that:

1. The image of $c_{i}$, for $i$ odd, is contained in the compact set $K$. Moreover at points belonging to these segments the absolute value of $\omega_{1}$ is bounded from below, i.e., $\left|\omega_{1}\right| \geq \alpha>0$.

2. If $i_{0}$ is odd, then the sum of the lengths of all even $i, i<i_{0}$, is less than, say, $2 / 3$ the sum of the lengths of $c_{1}, \ldots, c_{i_{0}}$.

3. The absolute value of the coordinate $z$ decreases monotonically along the segment $c_{p}$.

Fix $q \in \mathcal{P}_{\infty}^{-1}(p)$ and let $L$ be the leaf through $q$. Consider the lift of $l_{p}$ to $L$ and denote it by $l_{q}$. Note that $l_{q}$ is an oriented trajectory of $\mathcal{H}$ over $L$. We want to express $l_{q}$ in the corresponding affine coordinates $(x, y, z)$. More precisely, our goal will be to compute the value of its last coordinate $z$. For this, consider a connected oriented path $c$ contained in $l_{p}$ and joining $p$ to another point of $l_{p}$. Consider also a lift of $c$ contained in $l_{q}$. The $z$-coordinate of the mentioned lift is given by $z=z_{0} \exp \left[-\int_{c} \omega_{1}\right]$ where $z_{0}$ is the $z$-coordinate of $q$. In other words, $z_{0}$ is the height of $q$ relative to $L_{\infty}$. In particular

$$
\begin{aligned}
|z| & =\left|z_{0} e^{-\int_{c} \omega_{1}}\right|=\left|z_{0}\right| e^{-\operatorname{Re} \int_{c} \omega_{1}}=\left|z_{0}\right| e^{-\int_{0}^{1} \operatorname{Re}\left(\omega_{1}(c(t)) \cdot c^{\prime}(t)\right) d t} \\
& =\left|z_{0}\right| e^{-\int_{0}^{1} \mid\left(\omega_{1}(c(t)) \cdot c^{\prime}(t) \mid d t\right.} \leq\left|z_{0}\right| e^{-\int_{0}^{1} \alpha\left|c^{\prime}(t)\right| d t / 3}=\left|z_{0}\right| e^{-\alpha \operatorname{length}(c) / 3}
\end{aligned}
$$

This estimate shows the following: whenever a segment of $l_{p}$ having length equal to $3 \ln (2) / 2 \alpha$ is lifted to a regular leaf of $\widetilde{\mathcal{F}}$ projecting to $L_{\infty}$, the height of the final point of the lift in question is at most $1 / 2$ of the height of its initial point. 
Now the integral $\int_{l_{q}} d T$ can be estimated as follows. The time-form on $L$ is given in local coordinates by $d T=z^{d-1} d x / F(x, y)$. Since $l_{p}$, the image of $l_{q}$ by $\mathcal{P}_{\infty}$, is contained in a compact set not intersecting the singular set of $\widetilde{\mathcal{F}}_{\infty}$, the absolute value of $F(x, y)$ is bounded from below, i.e., $|F(x, y)| \geq \beta>0$ for all $(x, y) \in \Delta_{\infty} \backslash W$. Otherwise we replace $F$ by $G$ (recall that we are dealing only with regular points of $\widetilde{\mathcal{F}}$ on $\left.\Delta_{\infty}\right)$. Hence, considering $l_{q}$ as the concatenation of segments having length equal to $3 \ln (2) / 2 \alpha, l_{q}=\sum_{i=0}^{\infty} l_{i, q}$, it follows that

$$
\begin{aligned}
& \left|\int_{l_{q}} d T\right|=\left|\sum_{i=0}^{\infty} \int_{l_{i, q}} \frac{z^{d-1}}{F(x, y)} d x\right| \leq \sum_{i=0}^{\infty}\left|\int_{0}^{1} \frac{z_{i, q}^{d-1}(t)}{F\left(x_{i, q}(t), y_{i, q}(t)\right)} x_{i, q}^{\prime}(t) d t\right| \\
& \quad \leq \sum_{i=0}^{\infty} \int_{0}^{1} \frac{\left|z_{i, q}(t)\right|^{d-1}}{\left|F\left(x_{i, q}(t), y_{i, q}(t)\right)\right|}\left|x_{i, q}^{\prime}(t)\right| d t \leq \sum_{i=0}^{\infty} \int_{0}^{1} \frac{\left|z_{0}\right|^{d-1}(1 / 2)^{i(d-1)}}{\beta}\left|l_{i, p}^{\prime}(t)\right| d t \\
& \quad \leq \frac{\left|z_{0}\right|^{d-1}}{\beta} \operatorname{length}\left(l_{i, p}\right) \sum_{i=0}^{\infty}\left(\frac{1}{2^{d-1}}\right)^{i}=\frac{3\left|z_{0}\right|^{d-1} \ln (2)}{2 \alpha \beta} \frac{1}{1-(1 / 2)^{d-1}}<\infty,
\end{aligned}
$$

where $l_{i, q}(t)=\left(x_{i, q}(t), y_{i, q}(t), z_{i, q}(t)\right), t \in[0,1]$, is such that $l_{q}=\sum_{i=0}^{\infty} l_{i, q}$ and $\mathcal{P}_{\infty}\left(l_{i, q}\right)=l_{i, p}$. The theorem follows.

What precedes shows that the above mentioned integral is, indeed, bounded on $\Delta_{\infty} \backslash W$. Our next goal is to remove the condition on $W$; i.e., we want to allow the trajectory $l_{p}$ (resp. $l_{p}^{\theta}$ ) to accumulate on the singular set of $\widetilde{\mathcal{F}}$ in $\Delta_{\infty}$. This will lead us to study the behavior of this integral over segments of trajectories of $\mathcal{H}$ (resp. $\mathcal{H}^{\theta}$ ) that are close to the singularities of $\widetilde{\mathcal{F}}$. This local analysis will be the object of Section 5. Nonetheless, to finish the current section, we give an elementary general result concerning trajectories of $\mathcal{H}$ and of $\mathcal{H}^{\theta}$ that are contained in a local separatrix for a singularity of $\widetilde{\mathcal{F}}$ or $\widetilde{\mathcal{F}}_{\infty}$ in the particular case where $\widetilde{\mathcal{F}}$ is associated with a semi-complete vector field $\widetilde{X}$ satisfying also the preceding conditions. This goes as follows.

Consider again a vector field $\widetilde{X}$ as in (4.1). Let $p \in \Delta_{\infty}$ be a singular point of $\widetilde{\mathcal{F}}$ and consider an (germ of) analytic curve $\operatorname{Sep} \subset \Delta_{\infty}$ passing through $p$, invariant under $\widetilde{\mathcal{F}}_{\infty}$ and not entirely contained in the singular set of $\widetilde{\mathcal{F}}$. Let $\gamma(t)$ denote a local, irreducible, Puiseaux parametrization of Sep defined on a neighborhood of $0 \in \mathbb{C}$. Denote by $f(t) \frac{\partial}{\partial t}$ the pullback under $\gamma$ of the restriction of the vector field $F(x, y) \partial / \partial x+G(x, y) \partial / \partial y$ to Sep. Denote also by $h=h(t)$ the function $t \mapsto H \circ \gamma(t)$. Then, the pullback of the restriction of $\widetilde{X}$ to the cone over Sep is given by

$$
\widetilde{X}_{S}=z^{1-d}\left[f(t) \frac{\partial}{\partial t}+z h(t) \frac{\partial}{\partial z}\right] .
$$

Denote by $k$ (resp. $l$ ) the order of $f($ resp. $h$ ) at $0 \in \mathbb{C}$. Now we have:

Lemma 4.8. Assume that $\widetilde{X}$, as in Equation (4.1), is semi-complete and consider the vector field $\tilde{X}_{S}$ along with integers $k$ and $l$ as above. Then $l \geq k-1$ and the nature of $\omega_{1}$ (restricted to Sep) at $p$ is determined by the relation between $k$ and $l$. More precisely, the following hold: 
- If $l>k$ then $\omega_{1}$ is holomorphic and the restriction of $\mathcal{H}$ to Sep has a saddle singularity at $p$ with $2 m$ separatrices (for a certain $m \geq 1$ ).

- If $l=k$ then $\omega_{1}$ is regular at $p$ (and, in particular, holomorphic).

- If $l=k-1$ then $\omega_{1}$ has a simple pole at $p$. The residue of this pole is equal to $\alpha=-\left(h / f^{\prime}\right)(0)$. Then the restriction of $\mathcal{H}$ to Sep has a sink (resp. source) at $p$ provided that $\alpha \in \mathbb{R}_{+}$(resp. $\left.\alpha \in \mathbb{R}_{-}\right)$.

According to [29], see also the beginning of the present section, the vector field $\widetilde{X}_{S}$ induces an affine structure on $\{z=0\}$. In addition, this affine structure can be compared with the standard Euclidean structure to yield a 1-form $\beta$ called the affine defect of the former affine structure (see Section 3 of [29]). In the present case, the 1 -form $\beta$ is simply

$$
\beta=\left(\frac{-f^{\prime}}{f}+(d-1) \frac{h}{f}\right) d t .
$$

Proof of Lemma 4.8. The argument given here relies on part of the theory developed in [29]. Keep the preceding notations and suppose that $X$ is semi-complete. Since $X$ is semi-complete, the affine structure induced by $X$ on $\{z=0\}$ is uniformizable, cf. [29]. In turn, according to Proposition 6 of [29], the fact that the affine structure in question is uniform ensures that $\beta$ has at most a simple pole at $t=0$. Furthermore the residue associated to this simple pole has the form $-1+1 / n$ where $n \in \mathbb{Z}^{*} \cup\{\infty\}$. We also point out here that, when $n \in \mathbb{Z}^{*}$, the Fundamental Lemma proven in [29] ensures that the local holonomy map associated to the invariant axis $\{z=0\}$ has finite order dividing $n$.

Now assume that $\beta$ has only simple poles. Since the poles of $f^{\prime} / f$ are necessarily simple as well, we conclude that also $h / f$ can have at worst simple poles. In other words, we have proved that $l \geq k-1$ provided that $X$ is semi-complete.

Assume now that $l=k-1$ and denote by $\alpha \neq 0$ the residue of the (simple) pole of $h / f$ at $t=0$. It was seen that the residue of $\beta$ at $t=0$, if not zero, has the form $-1+1 / n$ and from this it follows that $\alpha$ is rational, and hence real, provided that $X$ is semi-complete. On the other hand, $\alpha$ is also the residue of $\omega_{1}$ at $t=0$. In particular, if $\alpha>0$ (resp. $\alpha<0$ ) then $\mathcal{H}$ has a sink (resp. source) at $t=0$.

To complete the proof of the lemma, suppose that $l \geq k$. It follows at once from the definition of $\omega_{1}$ that this form is holomorphic and nonzero at $t=0$ provided that $l=k$. Similarly, if $l>k$, then $\omega_{1}$ is still holomorphic at $t=0$. However, in this case, $t=0$ constitutes a zero of $\omega_{1}$. The corresponding consequences for the local behavior of $\mathcal{H}$ having already been known, the proof of the lemma is completed.

Remark 4.9. In the preceding argument, it should be emphasized that only the quotient $h / f^{\prime}$ at 0 must belong to $\mathbb{R}$ (in fact, to $\mathbb{Q}^{*}$ ) because $X$ is assumed to be semi-complete. Indeed, the residue of a simple pole for the 1 -form $\beta$ need not be $-1+1 / n$, with $n \in \mathbb{Z}^{*} \cup\{\infty\}$ unless the affine structure giving rise to $\beta$ is uniformizable. 
If the assumption that $X$ is semi-complete is dropped, then the foliations $\mathcal{H}$ and $\mathcal{H}^{\theta}$ may also admit singular points that behave as centers at the points corresponding to $t=z=0$ in the previous local coordinates $(t, z)$. This would add to the list of sink, source, and saddle singularities.

In closing we observe again that the preceding statements hold in arbitrary dimensions despite the fact that we have chosen to emphasize the 3-dimensional case. Details are left to the reader.

\section{The structure of $\mathcal{H}$ near singular points of $\widetilde{\mathcal{F}}_{\infty}$}

This section is devoted to establishing an extension of Theorem 4.7 allowing the trajectories of $\mathcal{H}$ and $\mathcal{H}^{\theta}$ to accumulate on singularities of $\widetilde{\mathcal{F}}$ and $\widetilde{\mathcal{F}}_{\infty}$. These singularities, however, will be supposed to be simple in the sense explained in the introduction. Here, it should be noted that the assumptions made on the structure of the singularities in question are not superfluous since certain saddle node singularities of a nature different from those considered in the introduction give rise to new complications preventing us from generalizing Theorem 4.7 without further information. On the other hand, the main result of this section, Theorem 5.1 below, remains valid for a large class of singular points; see the comments at the end of the section. As in Sections 3 and 4, we focus on the 3-dimensional case. The extensions of the arguments to higher dimensions, however, pose no further difficulty.

Throughout this section we shall deal with a homogeneous semi-complete vector field $X$ on $\mathbb{C}^{3}$ which, in addition, is assumed to have a singular set of codimension at least 2 . Also the degree $d$ of $X$ is assumed to satisfy $d \geq 2$.

We consider again the foliation $\widetilde{\mathcal{F}}$ associated to a homogeneous (polynomial) semi-complete vector field $X$ on $\mathbb{C}^{3}$ and assume that the singularities of $\widetilde{\mathcal{F}}$ lying in $\Delta_{\infty}$ are simple in the sense stated in the introduction. Recall that the foliation $\widetilde{\mathcal{F}}$ is tangent to the vector field $\widetilde{X}$ obtained from the initial vector field $X$ and given in the affine coordinates $(x, y, z)$ of Section 3 by (4.1). Namely, we have

$$
\widetilde{X}=\frac{1}{z^{d-1}}\left[F(x, y) \frac{\partial}{\partial x}+G(x, y) \frac{\partial}{\partial y}+z H(x, y) \frac{\partial}{\partial z}\right] .
$$

Suppose that $p \in \Delta_{\infty}$ is a singular point of $\widetilde{\mathcal{F}}$ and consider local coordinates $(u, v, w)$ about $p$, with $w$ locally equal to $z$. In these coordinates, a local representative for the foliation $\widetilde{\mathcal{F}}$ is provided by a vector field $Y$ having the form

$$
Y=\bar{F}(u, v) \frac{\partial}{\partial u}+\bar{G}(u, v) \frac{\partial}{\partial v}+w \bar{H}(u, v) \frac{\partial}{\partial w}
$$

for certain holomorphic functions $\bar{F}, \bar{G}$, and $\bar{H}$ with no nontrivial common factors, where $p$ is identified with the origin of $\mathbb{C}^{3}$. The singular point $p$ is then said to be simple (in the sense described in the introduction) if the linear part of $Z=\bar{F} \partial / \partial u+\bar{G} \partial / \partial v$ at $(0,0) \in \mathbb{C}^{2}$ possesses two eigenvalues different from zero. 
Moreover, when the quotient of these eigenvalues happens to be a positive integer, the induced foliation on $\Delta_{\infty}$ is linearizable (in other words, it is not conjugate to its Poincaré-Dulac normal form; see, for example, [4]). With these assumptions, Theorem 4.7 admits the following extension:

Theorem 5.1. Let $X$ be a homogenous polynomial semi-complete vector field whose singular set has codimension $\geq 2$. Assume that all the singularities of $\widetilde{\mathcal{F}}$ are simple (in the sense indicated in the introduction). Suppose that there is $\theta \in(-\pi / 2, \pi / 2)$ and a point $\mathrm{P} \in \Delta_{\infty}$ such that the trajectory $l_{\mathrm{P}}^{\theta}$ of $\mathcal{H}^{\theta}$ through $\mathrm{P}$ has infinite length. Then $\int_{l_{q}} d T$ converges for all $q \in \mathcal{P}_{\infty}^{-1}(\mathrm{P})$, where $l_{q}$ denotes the lift of $l_{\mathrm{P}}$ to the leaf through $q$ and $d T$ is the time form associated to $\widetilde{X}$.

This section is devoted to the proof of Theorem 5.1. Applications of Theorem 5.1, along with Theorem 4.7, will be worked out in Sections 6 and 7. Before proceeding, let us first revisit the statement of Theorem 5.1 to make its assumptions clear.

First, the singular set of $X$ has codimension $\geq 2$. This implies that $\bar{P}$ as in (4.2) is invertible. Therefore it can be assumed to be constant, equal to 1 . Thus, in the case where $X$ has degree $d \geq 3, P^{*}$ is invertible as well (see Corollary 4.4). The same does not necessarily occur for homogeneous (polynomial) vector fields of degree $d=2$. Nonetheless, since we are assuming that the singular points of $\tilde{\mathcal{F}}$ on $\Delta_{\infty}$ are simple, $P^{*}$ can also be supposed invertible (and therefore constant) even for $d=2$. So, from now on, the greatest common divisor of $F$ and $G$ in (5.1) is assumed to be 1, i.e., $P$ and $Q$ are relatively prime.

To begin, fix a point $p \in \Delta_{\infty}$ contained in the singular set of $\widetilde{\mathcal{F}}$. Recall that the two eigenvalues of $\widetilde{\mathcal{F}}_{\infty}$ at $p$ are assumed to be different from zero (and, when they are of the form $1, N$ with $N \in \mathbb{Z}_{+}$, it is also assumed that $\widetilde{\mathcal{F}}_{\infty}$ is not conjugate to its Poincaré-Dulac normal form). To be more precise, let $Y$ be the vector field in (5.2) with singular set of codimension at least 2 and tangent to $\widetilde{\mathcal{F}}$. The conditions on the singularities of $\widetilde{\mathcal{F}}$ and $\widetilde{\mathcal{F}}_{\infty}$ imply that $\operatorname{gcd}(\bar{F}, \bar{G})=1$. They also imply that the vector field $Z=\bar{F} \partial / \partial u+\bar{G} \partial / \partial v$ has eigenvalues $\lambda_{1}$ and $\lambda_{2}$ at $(0,0) \simeq p$ with $\lambda_{1} \lambda_{2} \neq 0$. Furthermore if the pair $\lambda_{1}, \lambda_{2}$ has the form $1, N$ with $N \in \mathbb{Z}_{+}$, then $Z$ is linearizable (recalling that a nonlinearizable vector field satisfying the preceding conditions must be conjugate to $\left.\left(N x+y^{N}\right) \partial / \partial x+y \partial / \partial y\right)$. This summarizes the assumption of Theorem 5.1.

Comparing the expressions for the vector fields $\widetilde{X}$ and $Y$, given respectively in (5.1) and (5.2), it immediately follows that $H(p)=\bar{H}(0,0)$.

Now we state the following:

Lemma 5.2. Fix a separatrix Sep for $\widetilde{\mathcal{F}}_{\infty}$ at a (simple) singular point $p_{0} \in \Delta_{\infty}$. Assume that $H(p)=\bar{H}(0,0)=0$. Then the Abelian form $\omega_{1}$ on the cone over Sep is holomorphic.

Proof. Note that the above mentioned vector field $Z$ representing $\widetilde{\mathcal{F}}_{\infty}$ on a neighborhood of $p$ has, by assumption, a linear part with two eigenvalues $\lambda_{1}$ and $\lambda_{2}$ different from zero. Suppose that $S e p$ is a (possibly singular) irreducible local 
separatrix for $\widetilde{\mathcal{F}}_{\infty}$ at $p$ and denote by $\gamma$ an irreducible Puiseaux parametrization for $S e p$. Since $\lambda_{1} \lambda_{2} \neq 0$, it is immediate that the order $k$ at $0 \in \mathbb{C}$ of the one-dimensional vector field obtained by pulling-back the restriction of the vector field $Z$ to $S e p$ by $\gamma$ equals 1 . The statement then follows from Lemma 4.8.

It follows from Lemma 5.2 that $p$ is either a regular point or a saddle singularity for $\mathcal{H}$ provided that $H(p)=0$. In view of the discussion at the end of Section 3 (about the global definition of $\mathcal{H}$-trajectories), singular points of saddle-type for $\mathcal{H}$ do not yield endpoints for any trajectory of $\mathcal{H}$. Indeed, every trajectory of $\mathcal{H}$ entering a small neighborhood of the singular point in question will eventually leave this same neighborhood. Moreover, as already shown, from a global point of view every trajectory of $\mathcal{H}$ gives rise to a contracting holonomy map in the appropriate sense.

Whereas Lemma 5.2 does not require the vector field $X$ to be semi-complete, this assumption definitely plays a role in our next lemma concerning the case $H(p)=\bar{H}(0,0) \neq 0$. Note that this lemma already appears in [25].

Lemma 5.3. Suppose that the initial homogeneous polynomial vector field $X$, with degree $d \geq 2$, is semi-complete. Suppose also that $H(p)=\bar{H}(0,0) \neq 0$. Then $d=2$. Furthermore the ratios $\lambda_{1} / \bar{H}(0,0)$ and $\lambda_{2} / \bar{H}(0,0)$ are both integers (and hence real).

Proof. Consider the restriction of $\widetilde{X}$ to the invariant manifold $\{u=0, v=0\}$. This restriction is a semi-complete vector field that does not vanish identically, since $\bar{H}(0,0) \neq 0$. By noting that this restricted vector field is nothing but $\bar{H}(0,0) w^{2-d} \partial / \partial w$, the semi-complete assumption implies that $d=2$.

Since the singular points of $\widetilde{\mathcal{F}}$ in $\Delta_{\infty}$ are supposed to be simple, the foliation $\widetilde{\mathcal{F}}_{\infty}$ has at least two smooth separatrices through $(0,0) \simeq p$. Without loss of generality, these separatrices may be supposed to coincide with the axes $u$ and $v$. To prove that $\lambda_{1} / \bar{H}(0,0) \in \mathbb{Z}^{*}$, consider the restriction of $\widetilde{\mathcal{F}}$ to the 2 -plane sitting over the separatrix $\operatorname{Sep}=\{v=w=0\}$ of $\widetilde{\mathcal{F}}_{\infty}$. Clearly this 2-plane is invariant under $\widetilde{\mathcal{F}}$ and locally parameterized by the coordinates $u$ and $w$. The restriction $\widetilde{X}_{\mid}$ of $\widetilde{X}$ to the 2-plane in question expressed in $(u, w)$-coordinates is given simply by $\widetilde{X}_{\mid}=w^{1-d}[\bar{f}(u) \partial / \partial u+w \bar{h}(u) \partial / \partial w]=w^{-1}[\bar{f}() \partial / \partial u+w \bar{h}(u) \partial / \partial w]$, since $d=2$

On the other hand, the vector field $\widetilde{X}_{\mid}$is semi-complete on a neighborhood of the origin. Furthermore $\bar{h}(0)=\bar{H}(0,0) \neq 0$ while $\bar{f}(u)=\lambda_{1} u+\cdots$. In particular $\bar{f}(0)=0$. From this it follows that the axis $\{u=0\}$ is invariant under $\tilde{X}_{\mid}$and that the restriction of $\widetilde{X}_{\mid}$to this axis is a regular one-dimensional vector field. Since this restriction is regular and $\widetilde{X}_{\mid}$is semi-complete, it follows that the local holonomy map associated to the axis in question must coincide with the identity (cf. the discussion in the proof of Proposition 4.2). Since $\bar{f}(u)=\lambda_{1} u+\cdots$ an elementary calculation shows that the above mentioned holonomy map cannot coincide with the identity unless $\lambda_{1} / \bar{H}(0,0)$ is an integer. The case of $\lambda_{2} / \bar{H}(0,0)$ being analogous, the proof of the lemma is complete. 
Summarizing, both quotients $\lambda_{1} / \bar{H}(0,0)$ and $\lambda_{2} / \bar{H}(0,0)$ are nonzero integers when $X$ is semi-complete. In particular, the quotient of the eigenvalues of $\widetilde{\mathcal{F}}_{\infty}$ at the singular point $p_{0}$ is a rational number since it is given by $\lambda_{1} / \lambda_{2}$. Because we are treating the case $\bar{H}(0,0) \neq 0$ where the 1 -form $\omega_{1}$ has a simple pole at the origin $(\simeq p)$, there follows the existence of two different cases according to whether $\lambda_{1} / \lambda_{2} \in \mathbb{Q}_{+}$or $\lambda_{1} / \lambda_{2} \in \mathbb{Q}_{-}$. The first possibility can easily be treated.

Lemma 5.4. Let $X$ be as in the statement of Lemma 5.3. With the preceding notations suppose that $\lambda_{1} / \lambda_{2} \in \mathbb{Q}^{+}$. Then $p$ is a sink (resp. source) singularity for $\mathcal{H}$ provided that $\lambda_{1} / \bar{H}(0,0)>0$ (resp. $\left.\lambda_{2} / \bar{H}(0,0)<0\right)$. In both cases, p yields an endpoint for the trajectories of $\mathcal{H}$.

Proof. It suffices to consider the case $\lambda_{1} / \bar{H}(0,0)>0$. Clearly the structure of $\mathcal{H}$ over the two (smooth) separatrices of $\widetilde{\mathcal{F}}_{\infty}$ at $p$ corresponds to sinks. As to the remaining leaves, recall that they all accumulate at the origin. Furthermore the structure of $\mathcal{H}$ at regular points of these leaves has to have the same nature as the corresponding structure over the smooth separatrices. Thus all these trajectories point inward at the singularity $p \simeq(0,0)$. The lemma is proved.

The next step is to consider the case in which $\lambda_{1} / \lambda_{2} \in \mathbb{Q}_{-}$. The restriction of $\widetilde{\mathcal{F}}_{\infty}$ to a neighborhood of $p$ admits exactly 2 separatrices. These separatrices are the unique leaves (of the restriction of $\widetilde{\mathcal{F}}_{\infty}$ to a neighborhood of $p$ ) accumulating radially at the singular point $p$. In vague terms, the separatrices are the only leaves of $\widetilde{\mathcal{F}}_{\infty}$ accumulating at $p$ if we ignore the effect of the local holonomy of this foliation. Denote by Sep one of the separatrices, for example the separatrix associated to the eigenvalue $\lambda_{1}$. The restriction of $\mathcal{H}$ to Sep can have a singular point at $p \in$ Sep. The nature of this singular point depends also on the sign of the quotient $\lambda_{1} / \bar{H}(0,0)$. If $\lambda_{1} / \bar{H}(0,0)>0$ then $p$ corresponds to a sink of $\mathcal{H}$ (or of $\omega_{1}$ by a minor abuse of notation) over Sep. Conversely, in the case where $\lambda_{1} / \bar{H}(0,0)<0$, the singular point corresponds to a source. We note, however, that $\lambda_{1} / \bar{H}(0,0)$ and $\lambda_{2} / \bar{H}(0,0)$ have opposite signs. This implies that if $p$ is a sink of $\omega_{1}$ for one of the separatrices then $p$ is a source for the other.

The above indicated issue about source and sink singularities appearing on the two separatrices of a singularity $p$ as before deserves further comment. First, if we consider real trajectories of $\mathcal{H}$ in the separatrix admitting $p$ as a sink, then these trajectories will reach a future endpoint at $p$. Somehow compensating the existence of this future endpoint, new $\mathcal{H}$-trajectories are issued in the other separatrix. These phenomena can, however, occur for only finitely many leaves of our foliation since each separatrix of a singularity as above can give rise to only one global leaf of $\widetilde{\mathcal{F}}$ or $\widetilde{\mathcal{F}}_{\infty}$. In particular this will play no significant role in the proof of any of the theorems stated in the introduction. In this regard, a far more important observation concerns those $\mathcal{H}$-trajectories whose projection on $\Delta_{\infty}$ enters a small neighborhood of $p$ but that are not contained in the corresponding separatrix of $p$. In fact, these trajectories can naturally be continued through the saddle associated with the singularity of $\widetilde{\mathcal{F}}_{\infty}$ so as to eventually leave a fixed neighborhood of $p$. Indeed, the foliation $\mathcal{H}$ is regular over all leaves of $\widetilde{\mathcal{F}}_{\infty}$ different from the two 
separatrices on a neighborhood of $p$. Furthermore, as we are going to see next, the continued trajectory keeps the contractive character of its holonomy.

Suppose then that the eigenvalues $\lambda_{1}$ and $\lambda_{2}$ at $p$ satisfy $\lambda_{1} / \lambda_{2} \in \mathbb{Q}_{-}$. We continue to assume that $\bar{H}(0,0) \neq 0$ so that it can be normalized to be 1 . Let $U_{\varepsilon}=\{(x, y, z):|x|,|y| \leq \varepsilon\}$ be a small neighborhood of the origin $(0,0) \simeq p$, not containing other singular points of $\widetilde{\mathcal{F}}_{\infty}$. Fix a regular leaf $L_{\infty} \subseteq \Delta_{\infty}$ (distinct from the separatrices through $p$ ) intersecting $U_{\varepsilon}$ and consider a real trajectory $l \subseteq L_{\infty}$ for $\mathcal{H}$. For these singularities we have:

Proposition 5.5. Let $X$ be as in the statement of Lemma 5.3 and assume that $\lambda_{1} / \lambda_{2} \in \mathbb{R}_{-}$. Let $U_{\varepsilon}$ be a small neighborhood of the (simple) singular point $p \simeq 0$ as above. Then the integral $\int_{l_{q} \cap U_{\varepsilon}} d T$ is uniformly bounded for every $\mathrm{P} \in l$ and $q \in \mathcal{P}_{\infty}^{-1}(\mathrm{P})$.

Remark 5.6. It should be emphasized that the trajectory $l_{q}$ in the statement is viewed as a global trajectory of $\mathcal{H}$. In other words, the intersection $l_{q} \cap U$ has, in general, infinitely many connected components. Proposition 5.5, indeed, claims that the sum of the integrals of $d T$ over all these connected components is uniformly bounded.

Proof of Proposition 5.5. Let $X$ be as in the statement of Lemma 5.3. Since $\bar{H}(0,0) \neq 0$, we can assume that $\bar{H}(0,0)=1$. Also, it follows that the degree $d$ of $X$ is 2 (Lemma 5.3). Nonetheless to help the reader with the discussion conducted immediately after the end of the proof of Proposition 5.5 (see the appendix to Section 5 ), we shall denote this degree by $d$ and only make the substitution $d=2$ at the very end of the proof.

According to Lemma 5.3, both eigenvalues $\lambda_{1}$ and $\lambda_{2}$ must be integers so that $\lambda_{1} / \lambda_{2}$ belongs to $\mathbb{Q}$. By assumption, this quotient must, in fact, belong to $\mathbb{Q}_{-}$, i.e., $\lambda_{1}$ and $\lambda_{2}$ have opposite signs. Next consider the foliation $\widetilde{\mathcal{F}}_{\infty}$ induced on $\Delta_{\infty}$. Since $\lambda_{1} / \lambda_{2} \in \mathbb{Q}_{-}$, the corresponding singular point $p$ admits two (smooth) separatrices. In local coordinates $(u, v, w)$ centered at $p$ as before, these separatrices can be identified with the axes $\{u=0\}$ and $\{v=0\}$. Thus, if $(u, v, w)$ are suitably chosen, the vector field $\widetilde{X}$ takes the (local) form

$$
\widetilde{X}=w^{1-d}\left[\bar{F}(u, v) \frac{\partial}{\partial u}+\bar{G}(u, v) \frac{\partial}{\partial v}+w \bar{H}(u, v) \frac{\partial}{\partial w}\right]
$$

where $\bar{F}(u, v)=u\left(\lambda_{1}+\right.$ h.o.t. $), \bar{G}(u, v)=v\left(\lambda_{2}+\right.$ h.o.t. $), \lambda_{1} / \lambda_{2} \in \mathbb{Q}$ - and $\bar{H}(0,0)=1$.

Assume, without loss of generality, that $\lambda_{1} \in \mathbb{R}_{+}$(resp. $\lambda_{2} \in \mathbb{R}_{-}$) and consider the restriction of $\omega_{1}$ to the $u$-axis (resp. $v$-axis). The residue of $\omega_{1}$ at $0 \simeq p$ with respect to this axis is equal to $-\bar{H}(0,0) / \lambda_{1}$ (resp. $\left.-\bar{H}(0,0) / \lambda_{2}\right)$. Therefore the restriction of $\mathcal{H}$ to the $u$-axis (resp. $v$-axis) has a sink singularity (resp. source singularity) at $p \simeq 0$. Hence, the real trajectories contained in the $u$-axis approach $p$. Similarly, those trajectories contained in the $v$-axis move away from $p$. It is easy to describe the behavior of $\mathcal{H}$ on the regular leaves of $U$ not accumulating at $p$ : over a real trajectory of $\left.\mathcal{H}\right|_{U}$ the absolute value of $u$ decreases while the absolute value 
of $v$ increases. In other words, a real trajectory moves away from the invariant plane $\{v=0\}$ while it approaches the plane $\{u=0\}$. In particular, whenever a (global) real trajectory $l$ enters the open set $U_{\varepsilon}$ it necessarily leaves $U_{\varepsilon}$ as well.

The preceding discussion shows that the only possibility for an $\mathcal{H}$-trajectory (not contained in the global leaves arising from the axes $\{v=w=0\}$ and $\{u=$ $w=0\}$ ) to accumulate at the singular point $p$ is when this trajectory enters the open set $U_{\varepsilon}$ infinitely many times. The sequence of points defined by the moment in which the trajectory enters $U_{\varepsilon}$ must also contain a subsequence that converges to a point in the $u$-axis. Also, in this case, it is immediate to check that the length of each connected component of $l \cap U_{\varepsilon}$ is bounded above by some uniform constant.

For each leaf of $\widetilde{\mathcal{F}} \cap U_{\varepsilon}$ not contained in the invariant plane $\{u=0\}$, the time-form is given by

$$
d T=\frac{w^{d-1}}{\bar{F}(u, v)} d u
$$

The leaf can be parameterized locally by the variable $u$ by means of the map $u \mapsto(u, v(u), w(u))$ where $w$ is given by (3.4). The expressions for $\bar{F}$ and $\bar{G}$ allow us to see that $v(u)=v_{0}\left(u / u_{0}\right)^{\lambda_{2} / \lambda_{1}} g(u)$ for some bounded holomorphic function $g$ on $\mathbb{C} \backslash \mathbb{R}_{-}^{*}$ satisfying $\lim _{u \rightarrow u_{0}} g(u)=1$. In turn, the coordinate $w$ is given by $w=w_{0} e^{-\int_{u_{0}}^{u} \omega_{1}}$, where $\omega_{1}$ coincides with $-\bar{H}(u, v(u)) / \bar{F}(u, v(u)) d u$. Therefore, substituting $v$ and $w$ in (5.3), we obtain

$$
d T=w_{0}^{d-1} \frac{1}{\bar{F}(u, v(u))} e^{-(d-1) \int_{u_{0}}^{u} \omega_{1}} d u .
$$

Since we need to estimate the integral of the time-form over oriented real trajectories of $\mathcal{H}$, let us start by controlling the exponential term. Since $\bar{H}(0,0)=1$, it follows that

$$
-\frac{\bar{H}(u, v)}{\bar{F}(u, v)}=-\frac{1}{\lambda_{1} u}(1+R(u, v))
$$

for some holomorphic function $R(u, v)$ satisfying $R(0,0)=0$ and defined on a neighborhood of the origin. In particular, if $\varepsilon$ is sufficiently small, the absolute value of $R(u, v)$ is bounded above on $U_{\varepsilon}$ by a small constant $0<\delta<<1$. If $l$ is a trajectory of $\mathcal{H}$ then $\int_{l} \omega_{1}$ is a positive real number. Therefore

$$
\left|e^{-(d-1) \int_{l} \omega_{1}}\right|=e^{-(d-1) \operatorname{Re} \int_{l} \omega_{1}}=e^{-(d-1) \int_{l} \omega_{1}} .
$$

Consider a (connected) segment of the real trajectory $l$ joining $u_{0}$ to $u$ where both points are contained in the neighborhood in question. Denote by $\phi:[0,1] \rightarrow L$ a parametrization of this segment satisfying $\phi(0)=u_{0}$ and $\phi(1)=u$. Up to a change of coordinates, close to a rotation, we can assume that the (connected) segment $\phi([0,1])$ is contained in the real axis. In fact, we can assume that it is contained in the positive component of the real axis. In particular, we can take 
$\phi(t)=u_{0}+t\left(u-u_{0}\right)$. It then follows that

$$
\begin{aligned}
\int_{l} \omega_{1} & =\int_{0}^{1} \omega_{1} \cdot \phi=\int_{0}^{1}-\frac{\phi^{\prime}(t)}{\lambda_{1} \phi(t)}(1+R(\phi(t), v(\phi(t))) d t \\
& =\frac{1}{\lambda_{1}} \int_{0}^{1}-\frac{u-u_{0}}{u_{0}+t\left(u-u_{0}\right)}(1+R(\phi(t), v(\phi(t))) d t \\
& \geq \frac{1-\delta}{\lambda_{1}} \int_{0}^{1}-\frac{u-u_{0}}{u_{0}+t\left(u-u_{0}\right)} d t=\frac{1-\delta}{\lambda_{1}} \ln \left(\frac{u_{0}}{u}\right) .
\end{aligned}
$$

Therefore we obtain

$$
\left|e^{-(d-1) \int_{l} \omega_{1}}\right| \leq C u^{(d-1)(1-\delta) / \lambda_{1}}
$$

where $C$ is a constant depending on $d, \lambda_{1}, \delta$, and $u_{0}(=\varepsilon)$. More accurately, $C=\varepsilon^{(1-d)(1-\delta) / \lambda_{1}}$. In fact, this estimate should be multiplied by a constant representing the supremum of the absolute value of the determinant of the change of coordinates. However we can, basically, include this quantity in $C$ since the absolute value of the determinant is bounded above on $U_{\varepsilon}$. Actually, the value of the determinant in question is very close to 1 since the change of coordinates is close to a rotation. In this sense, the constant $C$ does not depend on the segment of the real trajectory.

Now recall that $F(u, v)=\lambda_{1} u(1+r(u, v))$, for some holomorphic function $r$ on $U_{\varepsilon}$ satisfying $r(0,0)=0$. Up to reducing $\varepsilon$, we can assume that $|r(u, v)|$ is bounded above by a small constant $0<\tau<<1$. Therefore, the coefficient of the timeform satisfies

$$
|d T| \leq\left|w_{0}\right|^{d-1} \frac{C}{\lambda_{1}(1-\tau)} u^{(d-1)(1-\delta) / \lambda_{1}-1} .
$$

Since the exponent of $u$ is greater than -1 , the primitive of the coefficient of the time-form, up to the term $w_{0}^{d-1}$, is a bounded holomorphic function. It follows that the integral of the time-form, up the same term $w_{0}^{d-1}$, is bounded above on on each connected component $l_{i}$ of $l \cap U_{\varepsilon}$. In fact, there is a positive constant $K$, not depending on the trajectory of $\mathcal{H}$, such that

$$
\left|\int_{l_{i}} w_{0}^{1-d} d T\right|<K
$$

Finally the integral of the time form along $l_{i}$ is now bounded by $K$ times the absolute value of a positive power of the variable $w$ at the moment that the trajectory $l$ enters the open set $U_{\varepsilon}$ or, equivalently, at the starting point of $l_{i}$. We denote by $w_{i_{\sim}}$ the value $w$ at the starting point of $l_{i}$. As already mentioned, the holonomy of $\widetilde{\mathcal{F}}$ is contractive. Therefore, since the length of the real trajectory between two consecutive starting points of $l \cap U_{\varepsilon}$ is bounded from below, the sequence $w_{i}$ is such that $\left|w_{i+1}\right| /\left|w_{i}\right| \leq k$, for some constant $0<k<1$, since the trajectories of $\mathcal{H}$ have contractive holonomy. Thus

$$
\left|\int_{l \cap U_{\varepsilon}} d T\right| \leq \sum\left|\int_{l_{i}} d T\right| \leq \sum K\left|w_{i}\right|^{d-1} \leq \sum K\left|w_{0}\right|^{d-1} k^{i(d-1)}=\frac{K\left|w_{0}\right|^{d-1}}{1-k^{d-1}} .
$$


Since $d=2$, the last estimate ensures that the corresponding integral is uniformly bounded as desired.

Proof of Theorem 5.1. The proof follows immediately upon combining Theorem 4.7 with Proposition 5.5.

\section{Appendix to Section 5: a natural relaxation of the condition imposed on the singularities of $\widetilde{\mathcal{F}}$}

To close this section, we indicate a much weaker assumption on the singularities of $\widetilde{\mathcal{F}}$ that is still enough to yield Proposition 5.5, and hence Theorem 5.1. In fact, Proposition 5.5 can be extended to almost all of the class of singularities named absolutely isolated; see [12].

To explain this generalization, consider the vector field $\widetilde{X}$ given in local coordinates $(u, v, w)$ around a singular point of $\widetilde{\mathcal{F}}$ in $\Delta_{\infty}$ by

$$
\widetilde{X}=u^{n} v^{m} w^{1-d}\left[\bar{F}(u, v) \frac{\partial}{\partial u}+\bar{G}(u, v) \frac{\partial}{\partial v}+w \bar{H}(u, v) \frac{\partial}{\partial w}\right]
$$

where $d \geq 2$ and $n, m \in \mathbb{Z}$. Assume also that the singularity of the vector field $\bar{F}(u, v) \partial / \partial u+\bar{G}(u, v) \partial / \partial v$ at $(0,0) \in \mathbb{C}^{2}$ is simple and that $\bar{H}(0,0) \neq 0$. As stated, Proposition 5.5 no longer holds for all $\widetilde{X}$ as above. However, it still holds for $\widetilde{X}$ as above under the additional assumption that $\max \{m, n\} \leq 0$, as can straightforwardly be checked from the above given proof of Proposition 5.5 (this explains why we made the substitution $d=2$ only at the end of the corresponding proof).

The preceding observation is the key to adapting Theorem 5.1 and, by means of it, also Theorem B) to a much larger class of vector fields having absolutely isolated singularities, as opposed to simple singularities, on the hyperplane at infinity $\Delta_{\infty}$. This goes as follows. Denote again by $X$ a polynomial vector field whose associated foliation $\mathcal{D}$ has only absolutely isolated singularities on $\Delta_{\infty}$. Suppose also that the singular set of $X$ has codimension at least 2. According to the main result of [12], these singularities can be reduced by applying to them successive pointwise blow-ups. Furthermore, if the very generic assumption that the absence of singularities of saddle-node type in the reduction procedure is added, then the final (reduced) singularities will be of the type appearing in (5.6). Moreover, in the vast majority of cases, the corresponding integers $m$ and $n$ are nonpositive. Thus, for the corresponding vector fields, Theorem 5.1 will still hold.

Summarizing what precedes:

1. Proposition 5.5, and hence Theorem 5.1, cannot be extended to arbitrary singular points without additional information on the global dynamics of the foliation $\widetilde{\mathcal{F}}_{\infty}$ on $\Delta_{\infty}$.

2. This proposition, however, can be extended to a vast class of singular points that is generic among singular points for any priori fixed order. In particular, in the class of singular points for which Proposition 5.5 (and Theorem 5.1) still holds, there can be found singularities exhibiting some very complicated dynamical behavior. 


\section{Applications to complete vector fields}

\subsection{Ends of solutions of complete polynomial vector fields on $\mathbb{C}^{n}$}

This first application concerns Theorem 4.7. Consider a complete polynomial vector field $X$ defined on $\mathbb{C}^{n}$. Set $X=\sum_{i=0}^{d} X_{i}$ where $X_{i}$ stands for the homogeneous component of degree $i$ of $X$. To maintain as much as possible the notation used in previous sections, the foliation associated with $X$ will be denoted by $\mathcal{D}$ whereas $\mathcal{F}$ will stand for the foliation associated to the top degree homogeneous component $X_{d}$. Throughout what follows, the degree $d$ is assumed to be at least 2 .

Recall that both foliations $\mathcal{D}$ and $\mathcal{F}$ admit holomorphic extensions to $\mathbb{C P}^{n}$ and these extensions are also denoted by $\mathcal{D}$ and $\mathcal{F}$.

Lemma 6.1. The homogeneous vector field $X_{d}$ is not a multiple of the radial vector field

$$
R=x_{1} \partial / \partial x_{1}+\cdots+x_{n} \partial / \partial x_{n}
$$

Proof. First note that the vector field $X_{d}$ is semi-complete on all of $\mathbb{C}^{n}$ since it is the top degree homogeneous component of a complete vector field. More precisely, let $\Lambda_{n}$ denote the map $\left(x_{1}, \ldots, x_{n}\right) \mapsto\left(2^{n} x_{1}, \ldots, 2^{n} x_{n}\right)$. Next let $Y_{n}$ be the vector field defined by $2^{(1-d) n} \cdot \Lambda_{n}^{*} X$. For every fixed $n, Y_{n}$ is clearly a complete vector field on $\mathbb{C}^{n}$ so that its restriction to every open set of $\mathbb{C}^{n}$ is semi-complete. Furthermore the sequence $\left\{Y_{n}\right\}$ converges uniformly on compact sets to the vector field $X_{d}$. Since the set of semi-complete vector fields is closed in the topology of uniform convergence on compact sets, it follows that $X_{d}$ is semi-complete on all of $\mathbb{C}^{n}$.

Suppose now that $X_{d}$ is a multiple $f R$ of $R$. In view of Lemma 2.6, it follows that $f$ is a linear form, i.e., a homogeneous degree 1 polynomial. To obtain a contradiction with this last possibility, we proceed as follows. First, note that the generic leaf $L$ of $\mathcal{D}$ intersects the hyperplane at infinity of $\mathbb{C P}^{n}$ transversely at a regular point $p$ of $\mathcal{D}$. Moreover the point $p$ is regular for the restriction of $X$ to $L$. In other words, the flow of $X$ reaches the hyperplane at infinity in finite time. This is impossible since $X$ is complete on $\mathbb{C}^{n}$. This concludes the proof of the lemma.

Again let $\Delta_{\infty}$ denote the hyperplane at infinity in $\mathbb{C P}^{n}$. It follows from the preceding that $\Delta_{\infty}$ is invariant under both $\mathcal{D}$ and $\mathcal{F}$. In addition, the foliations induced on $\Delta_{\infty}$ by $\mathcal{D}$ and $\mathcal{F}$ coincide. The foliation induced by $\mathcal{F}$ on $\Delta_{\infty}$ will be denoted by $\mathcal{F}_{\infty}$. Also $\Delta_{\infty}$ corresponds to the divisor of poles for both $X$ and $X_{d}$. Since the methods developed in the previous sections apply to foliations associated to homogeneous vector fields, they are in principle not applicable to $\mathcal{D}$ but only to $\mathcal{F}$. However, near $\Delta_{\infty}$, the foliation $\mathcal{D}$ becomes very close to $\mathcal{F}$. In the sequel we are going to combine these two observations in order to establish Theorem A.

We begin by choosing affine coordinates $\left(x_{1}, \ldots, x_{n-1}, z\right)$ analogous to those used in Sections 3 and 4. Namely the hyperplane $\{z=0\}$ is contained in $\Delta_{\infty}$ and the plane at infinity $\Delta_{\infty}^{1, \ldots, n-1}$, defined by the affine coordinates $x_{1}, \ldots, x_{n-1}$, where $z=0$ is fixed, is not invariant under the restrictions of either $\mathcal{D}$ or $\mathcal{F}$ to $\Delta_{\infty}$. 
Then we can apply the results of Section 4 to the foliation $\mathcal{F}$. In particular, the leaves of $\mathcal{F}$ are equipped with the (singular) real foliations $\mathcal{H}^{\theta}$ where $\theta$ is chosen in the interval $(-\pi / 2, \pi / 2)$. For the rest of this section, these foliations will be denoted by $\mathcal{H}_{\mathcal{F}}$ (resp. $\mathcal{H}_{\mathcal{F}}^{\theta}$ ). To define a suitable version of these real trajectories in the leaves of $\mathcal{D}$ we proceed as follows. Given a point $p=\left(x_{1}^{0}, \ldots, x_{n-1}^{0}, z^{0}\right)$ with $z^{0} \neq 0$, let $L_{p}$ denote the leaf of $\mathcal{D}$ through $p$. To define the foliation $\mathcal{H}_{\mathcal{D}}$ at $p$, we consider the function $\left(x_{1}, \ldots, x_{n-1}, z\right) \mapsto|z|$ restricted to $L_{p}$. The tangent vector to $\mathcal{H}_{\mathcal{D}}$ at $p$ is simply the negative of the gradient of this function. Once $\mathcal{H}_{\mathcal{D}}$ is defined the foliations $\mathcal{H}_{\mathcal{D}}^{\theta}$ have an obvious definition since the leaves of $\mathcal{D}$ have natural conformal structures.

The next step in our construction consists of investigating the basic properties of $\mathcal{H}_{\mathcal{D}}$ in analogy with the properties of $\mathcal{H}_{\mathcal{F}}$ considered in Sections 3 and 4. Recalling that $\mathcal{D}$ and $\mathcal{F}$ induce the same foliation $\mathcal{F}_{\infty}$ on $\Delta_{\infty}$, consider a point $\left(x_{1}^{0}, \ldots, x_{n-1}^{0}, 0\right) \in \Delta_{\infty}$ that is regular for the restrictions of both $\mathcal{D}$ and $\mathcal{F}$ to $\Delta_{\infty}$. Then we have:

Lemma 6.2. The direction of $\mathcal{H}_{\mathcal{D}}$ at the point $\left(x_{1}^{0}, \ldots, x_{n-1}^{0}, z\right)$ converges uniformly to the direction of $\mathcal{H}_{\mathcal{F}}$ at $\left(x_{1}^{0}, \ldots, x_{n-1}^{0}, 0\right)$. In particular the foliation $\mathcal{H}_{\mathcal{D}}$ can be extended to the regular part of $\mathcal{D}$ in $\Delta_{\infty}$ and this extended foliation coincides with $\mathcal{H}_{\mathcal{F}}$ on $\Delta_{\infty}$.

Proof. Since the behavior of $\mathcal{D}$ near $\left(x_{1}^{0}, \ldots, x_{n-1}^{0}, 0\right)$ is dominated by the component $X_{d}$ of $X$, it suffices to check that the trajectories of $\mathcal{H}_{\mathcal{F}}$ admit a definition analogous to the one given above for the trajectories of $\mathcal{H}_{\mathcal{D}}$. In other words, it suffices to prove that the direction of $\mathcal{H}_{\mathcal{F}}$ at $\left(x_{1}^{0}, \ldots, x_{n-1}^{0}, z\right)$ coincides with the gradient of the function $\left(x_{1}, \ldots, x_{n-1}, z\right) \mapsto|z|$ restricted to the leaf of $\mathcal{F}$ through $\left(x_{1}^{0}, \ldots, x_{n-1}^{0}, z\right)$. This is, however, an immediate consequence of the formula (3.4). The lemma is proved.

Remark 6.3 (The trajectories $\mathcal{H}_{\mathcal{F}}$ and $\mathcal{H}_{\mathcal{D}}$ as geodesics for a foliated flat structure). By building on the proof of Lemma 6.2, we can provide further and more accurate information about separating curves and flat structure with bounded geometry as discussed in the introduction (after the statement of Theorem A'). In the case of homogeneous foliations, such as $\mathcal{F}$, every leaf $L$ of $\mathcal{F}$ is equipped with the 1 -form $\omega_{1}$ defined in Section 3. These include the leaves of $\mathcal{F}$ contained in $\Delta_{\infty}$ (or $\Delta_{0}$ ) so that $\omega_{1}$ is a foliated 1-form on the compact manifold $M$ which, in turn, ensures $\omega_{1}$ has bounded geometry. Since the leaves of $\mathcal{F}$ are Riemann surfaces, the restriction of $\omega_{1}$ to one of these leaves $L$ can equally well be seen as a singular flat structure on $L$. It is an elementary fact that, in suitable local coordinates adapted to this flat structure, the trajectories of $\mathcal{H}_{\mathcal{F}}^{\theta}$ (including $\mathcal{H}_{\mathcal{F}}$ and $\mathcal{H}_{\mathcal{F}}^{\frac{1}{\mathcal{F}}}$ ) are straight lines and hence geodesics for the flat structure itself. In particular, a trajectory of, say, $\mathcal{H}_{\mathcal{F}}^{\perp}$, satisfies all the conditions in the statement of Theorem A' for it to be a separating curve. In view of the preceding, it also follows that the flat structure for which these curves are geodesics has bounded geometry on the corresponding leaf of $\mathcal{F}$.

To extend the construction of the 1 -form $\omega_{1}$ to nonhomogeneous foliations such as $\mathcal{D}$, we proceed as follows. Consider a leaf $L$ of $\mathcal{D}$. We want to construct 
a 1 -form $\omega_{1, \mathcal{D}}$ on $L$ for which $\mathcal{H}_{\mathcal{D}}$ is the real foliation. For this, suppose that $L$ is not contained in $\Delta_{\infty}$ and consider a regular point $p \in L$ along with a vector $v \in T_{p} L$ the oriented in the direction of $\mathcal{H}_{\mathcal{D}}$. In particular, all directions for $\mathcal{H}_{\mathcal{D}}^{\theta}$ and $\mathcal{H}_{\mathcal{D}}^{\perp}$ are immediately defined through the conformal structure of $L$. To define $\omega_{1, \mathcal{D}}$ at $p$, we just need to associate a complex number with these real foliations. The complex number in question is simply the derivative of the holomorphic function $\left(x_{1}, \ldots, x_{n-1}, z\right) \mapsto z$ restricted to $L$. This construction equips every leaf $L$ of $\mathcal{D}$ not contained in $\Delta_{\infty}$ with an Abelian form $\omega_{1, \mathcal{D}}$ and hence with a singular flat structure. Finally, if $L$ is contained in $\Delta_{\infty}$, then we set $\omega_{1, \mathcal{D}}=\omega_{1}$ since the foliation $\mathcal{D}$ and $\mathcal{F}$ coincide on $\Delta_{\infty}$. The previous discussion show that $\omega_{1, \mathcal{D}}$ is a foliated 1-form for $\mathcal{D}$ defined on all of the compact manifold $M$. In this sense, the argument used in the case of $\omega_{1}$ can be repeated here to show that the foliated flat structure arising from $\omega_{1, \mathcal{D}}$ has bounded geometry. Moreover, the trajectories of $\mathcal{H}_{\mathcal{D}}^{\perp}$ define separating curves in the sense of Theorem A'.

To help us explain how to derive properties of $X$ and $\mathcal{D}$ from properties of $X_{d}$ and $\mathcal{F}$, it is convenient to consider a small neighborhood $V$ (in the $n$-dimensional ambient space) of $\left(\operatorname{Sing}(\mathcal{D}) \cap \Delta_{\infty}\right) \cup \operatorname{Sing}(X)$, where $\operatorname{Sing}(\mathcal{D})$ (resp. $\left.\operatorname{Sing}(X)\right)$ stands for the singular set of $\mathcal{D}$ (resp. $X$ ). Next, denote by $U$ a neighborhood of $\Delta_{\infty} \backslash V$ (not intersecting $\left.\left(\operatorname{Sing}(\mathcal{D}) \cap \Delta_{\infty}\right) \cup \operatorname{Sing}(X)\right)$. Also, in order to preserve the uniform contractive character along trajectories of $\mathcal{H}_{\mathcal{F}}^{\theta}$, we fix some (small) $\epsilon>0$ and consider $\theta$ in $(-\pi / 2+\epsilon, \pi / 2-\epsilon)$.

It follows from our general construction that the endpoints belonging to $U$ for trajectories of $\mathcal{H}_{\mathcal{F}}^{\theta}$ are situated over $\Delta_{\infty}^{1, \ldots, n-1}$. In particular, a trajectory of $\mathcal{H}_{\mathcal{F}}^{\theta}$ through an affine point $\left(x_{1}^{0}, \ldots, x_{n-1}^{0}, 0\right) \in U \cap \Delta_{\infty}$ never meets $\Delta_{\infty}^{1, \ldots, n-1}$, though it can pass arbitrarily close to $\Delta_{\infty}^{1, \ldots, n-1}$ as already discussed in Section 3 ; cf. however Lemma 3.10. Modulo choosing the neighborhood $U$ sufficiently narrow, the restriction to $U$ of the foliation $\mathcal{D}$ is very close to the (restriction to $U$ of the) foliation $\mathcal{F}$. A similar statement holds for the foliations $\mathcal{H}_{\mathcal{D}}$ and $\mathcal{H}_{\mathcal{F}}$ thanks to Lemma 6.2. In particular, we obtain the following:

Lemma 6.4. Let $\epsilon>0$ be fixed. Consider a point $p=\left(x_{1}^{0}, \ldots, x_{n-1}^{0}, z^{0}\right) \in U$ and denote by $l_{p}^{+, \theta}$ the semi-trajectory of $\mathcal{H}_{\mathcal{D}}^{\theta}$ started at $p$ for $\theta \in[-\pi / 2+\epsilon, \pi / 2-\epsilon]$. Consider a path $c:[0,1] \rightarrow l_{p}^{+, \theta} \cap U$, with $c(0)=p$, and set $c(1)=\left(x_{1}^{1}, \ldots, x_{n-1}^{1}, z^{1}\right)$. Then there is a constant Cte depending solely on $\epsilon$ such that the following condition is satisfied: whenever the length of $c$ exceeds Cte, we have the estimate $\left|z^{1}\right|<\left|z^{0}\right| / 2$.

Proof. This follows immediately from the proof of Theorem 4.7 concerning the foliation $\mathcal{F}$. More precisely, the statement was shown for $\mathcal{H}_{\mathcal{F}}$ but the adaptations needed for the foliations $\mathcal{H}_{\mathcal{F}}^{\theta}$ are clear. The present statement follows from the fact that inside $U$ the foliation $\mathcal{H}_{\mathcal{D}}^{\theta}$ is very close to $\mathcal{H}_{\mathcal{F}}^{\theta}$.

One last ingredient is still needed for the proof of Theorem A. Let $l_{p}^{+, \theta}$ be a trajectory as in Lemma 6.4 and denote by $L_{p}$ the leaf of $\mathcal{D}$ containing $l_{p}^{+, \theta}$. The idea behind of Theorem A consists of estimating the integral of $d T_{L}$ over $l_{p}^{+, \theta}$ 
where $d T_{L}$ stands for the time-form induced by $X$ on $L_{p}$. In Section 4 suitable estimates for this type of integral were obtained in the case of homogeneous vector fields. The estimate is based on the renormalized time-form induced on $\Delta_{\infty}$ by the vector field and on the evolution of the distance of the points to $\Delta_{\infty}$ (the height of the points). As to the heights of points, the preceding lemma provides a suitable control of their evolutions along trajectories of $\mathcal{H}_{\mathcal{D}}^{\theta}$ in the case of nonhomogeneous polynomial vector fields. Finally we recall that the foliations induced by $X$ and by $X_{d}$ on $\Delta_{\infty}$ turn out to coincide and the same holds for the renormalized timeforms induced on $\Delta_{\infty}$ by $X$ and by $X_{d}$.

We are now ready to prove Theorem A.

Proof of Theorem A. Consider the foliation $\mathcal{D}$ induced by $X$ on $\mathbb{C P}^{n}$ and let $\Delta_{\infty}$ be as above. Let $V$ denote the given neighborhood of $\left(\operatorname{Sing}(\mathcal{D}) \cap \Delta_{\infty}\right) \cup \operatorname{Sing}(X)$ and fix $\epsilon>0$. Next, choose a neighborhood $U$ of $\Delta_{\infty} \backslash V$ so that the statement of Lemma 6.4 holds. It is sufficient to prove the theorem for the foliation $\mathcal{H}_{\mathcal{D}}$ since the adaptations needed for the general case of the foliations $\mathcal{H}_{\mathcal{D}}^{\theta}, \theta \in(-\pi / 2+\epsilon, \pi / 2-\epsilon)$, are clear.

Consider a point $p=\left(x_{1}^{0}, \ldots, x_{n-1}^{0}, z^{0}\right) \in U \backslash V$. Denote by $l_{p}^{+}$(resp. $\left.L_{p}\right)$ the semi-trajectory of $\mathcal{H}_{\mathcal{D}}$ initiated at $p$ (resp. the leaf of $\mathcal{D}$ through $p$ ). Suppose first that $l_{p}^{+}$is contained in $U$. To explain the structure of the proof of our theorem, we shall first prove that the preceding assumption contradicts the completeness of $X$. For this, we are going to show that the integral of the time-form $d T_{L}$ induced by $X$ on $L_{p}$ over $l_{p}^{+}$converges. Since it clearly accumulates onto $\Delta_{\infty}$ (in particular $l_{p}^{+}$leaves every compact set contained in $L_{p}$ ) the convergence of the mentioned integral contradicts the completeness of $X$. We also note that our claim reduces to Theorem 4.7 in the case of homogeneous vector fields.

To adapt the proof of Theorem 4.7 to the present case where $X$ is not homogeneous we proceed as follows. The choice of the coordinates $\left(x_{1}, \ldots, x_{n-1}, z\right)=$ $(\underline{x}, z)$ allows us to write $X_{d}$ in the form

$$
X_{d}=z^{1-d}\left[F_{1}(\underline{x}) \partial / \partial x_{1}+\cdots+F_{n-1}(\underline{x}) \partial / \partial x_{n-1}+z H(\underline{x}) \partial / \partial z\right],
$$

while the vector field $X$ becomes

$$
X=z^{1-d}\left[F_{1}^{*}(\underline{x}, z) \partial / \partial x_{1}+\cdots+F_{n-1}^{*}(\underline{x}, z) \partial / \partial x_{n-1}+z H^{*}(\underline{x}, z) \partial / \partial z\right] .
$$

The coefficients $F_{i}$ and $F_{i}^{*}, i=1, \ldots, n-1,\left(\right.$ resp. $H_{i}$ and $\left.H_{i}^{*}\right)$ are related by the formulas

$$
F_{i}^{*}\left(x_{1}, \ldots, x_{n-1}, z\right)-F_{i}\left(x_{1}, \ldots, x_{n-1}\right)=z P_{i}\left(x_{1}, \ldots, x_{n-1}, z\right)
$$

(resp. $\left.H^{*}\left(x_{1}, \ldots, x_{n-1}, z\right)-H\left(x_{1}, \ldots, x_{n-1}\right)=z Q\left(x_{1}, \ldots, x_{n-1}, z\right)\right)$ where $Q$ and $P_{i}$ are polynomials in the variables in question. Next note that the time-form $d T_{L}$ is given by

$$
d T_{L}=\frac{z^{d-1}}{F_{1}^{*}\left(x_{1}, \ldots, x_{n-1}, z\right)} d x_{1}=\cdots=\frac{z^{d-1}}{F_{n-1}^{*}\left(x_{1}, \ldots, x_{n-1}, z\right)} d x_{n-1}
$$


Now since $U$ does not intersect the singular set of $X$ (or $\mathcal{D})$, we can suppose without loss of generality that $F_{1}^{*}\left(x_{1}, \ldots, x_{n-1}, z\right)$ is bounded from below by a positive constant $\beta$ in $U$, otherwise we replace $F_{1}^{*}$ by a suitable $F_{i}^{*}$. This last estimate combined with Lemma 6.4 then shows that the integral of $d T_{L}$ over $l_{p}^{+}$is bounded by simply repeating the calculations performed in the proof of Theorem 4.7.

We are then led to conclude that the semi-trajectory $l_{p}^{+}$must intersect the neighborhood $V$ of $\left(\operatorname{Sing}(\mathcal{D}) \cap \Delta_{\infty}\right) \cup \operatorname{Sing}(X)$ regardless of how small is $V$. In particular, it may happen that $l_{p}^{+}$accumulates at singular points of $\mathcal{D}$ lying in $\Delta_{\infty}$. In this case the integral of $d T_{L}$ over $l_{p}^{+}$cannot be bounded without additional conditions. Fortunately, in order to establish Theorem A, we do not need to keep track of the amount of time that $l_{p}^{+}$spends inside $V$ but rather of the amount of time that $l_{p}^{+}$spends away from $V$. To be more precise, let us prove the following:

Claim. The distance between the trajectory $l_{p}^{+}$and the hyperplane $\Delta_{\infty}$ cannot have a minimum unless this minimum is zero. Furthermore, when this minimum is zero, the intersection point $l_{p}^{+} \cap \Delta_{\infty}$ is never reached by the flow of $X$.

Before starting the proof of the claim, it is convenient to make some general comments regarding the possibility of having a point $q$ in $l_{p}^{+} \cap \Delta_{\infty}$. A first case where this may happen arises from the definition of "leaf" given in Section 2 and borrowed from [6]. According to this definition, the leaf $L_{p}$ of $\mathcal{D}$ may contain a singular point of $\mathcal{D}$ lying in $\Delta_{\infty}$. In fact, in this case, a local branch of $L_{p}$ about $q$ defines an irreducible separatrix for $\mathcal{D}$ at $q$. It is then natural to think of $q$ as belonging to $l_{p}^{+}$. More generally, it can happen that $l_{p}^{+}$converges to a point $q$ lying in $\Delta_{\infty}$ whether or not $q$ belongs to $L_{p}$. With a minor abuse of notation, the point $q$ may be thought of as belonging to $l_{p}^{+}$. In all these cases the statement of Theorem A is clear: the completeness of $X$ implies that the integral of $d T_{L}$ over a local branch of $l_{p}^{+}$converging to $q$ is infinite. So $l_{p}^{+}$enters every given neighborhood of $q$ and remains inside forever. The statement then follows from observing that $q$ must be a singular point of $\mathcal{D}$ since $\Delta_{\infty}$ is invariant by $\mathcal{D}$.

A further reduction in the proof of Theorem A is possible even though not strictly needed. Namely, with the above notation, we can suppose that (a local branch of) $l_{p}^{+}$never converges to a point $q$ that is singular for $X$ (and in particular for $\mathcal{D})$. In fact, if this point belongs $\Delta_{\infty}$ then the theorem results immediately as already seen. Similarly, if $q \in \operatorname{Sing}(X) \backslash \Delta_{\infty}$, then the theorem follows from the standard results on existence and uniqueness of solutions for regular ordinary differential equations.

Proof of the Claim. Given what precedes, we suppose, aiming at a contradiction, that $q$ is a point of minimum for the mentioned distance and that $q$ lies in $\mathbb{C P}^{n} \backslash \Delta_{\infty}$. First, we are going to prove that $q$ must belong to the domain of definition of the coordinates $\left(x_{1}, \ldots, x_{n-1}, z\right)$. Since $X$ is not homogeneous and $q$ is not in $\Delta_{\infty}$, this assertion is not an immediate consequence of Lemma 3.5. In order to prove it, suppose that $c:[0,1) \rightarrow l_{p}^{+}$is a local parametrization of $l_{p}^{+}$with $\lim _{t \rightarrow 1^{-}} c(t)=q$. Setting $c(t)=\left(x_{1}(t), \ldots, x_{n-1}(t), z(t)\right)$, it follows that $z(t)$ is locally bounded at $q$. Suppose, in addition, that $\left(x_{1}(t), \ldots, x_{n-1}(t)\right)$ leaves the domain of definition of coordinates $\left(x_{1}, \ldots, x_{n-1}, z\right)$. then, by using the standard 
coordinates of $\mathbb{C P}^{n}$ whose domain contains $\Delta_{\infty}$, the boundedness of $z(t)$ implies that $\lim _{t \rightarrow 1^{-}} c(t)=q$ belongs to $\Delta_{\infty}$. As already shown, the statement of the theorem holds in this situation.

Summarizing the above discussion, we can suppose that $q=\left(q_{1}, \ldots, q_{n}\right)$ is a regular point for $X$ and $\mathcal{D}$ belonging to the domain of definition of the coordinates $\left(x_{1}, \ldots, x_{n-1}, z\right)$ and satisfying $q_{n} \neq 0$. A final contradiction can now be obtained as follows. Let $\Phi(T)=\left(\Phi_{1}(T), \ldots, \Phi_{n}(T)\right)$ be a local parametrization of $L_{p}$ in a neighborhood of $q(\Phi(0)=q)$. Since $q$ is a regular point for $X$, the holomorphic map $T \mapsto \Phi_{n}(T) \in \mathbb{C}$ is not constant and hence it must be open which, in turn, contradicts the assumption that $\left|\Phi_{n}\right|$ has a (positive) local minimum at $T=0$. The claim is proved.

To finish the proof of Theorem A consider now the semi-trajectory $l_{p}^{+}$. The above discussion shows that $l_{p}^{+}$accumulates on $\Delta_{\infty}$, in particular $l_{p}^{+}$leaves compact sets of $L_{p}$. The completeness of $X$ then implies

$$
\int_{l_{p}^{+}} d T_{L}=\infty .
$$

Consider a decomposition $l_{p}^{+}=c_{1} * c_{2} * \cdots$ of $l_{p}^{+}$into finitely or infinitely many paths such that $c_{k}$ is contained in $U$ for $k$ odd and $c_{k}$ is contained in $V$ for $k$ even. The statement is now reduced to proving that

$$
\sum_{k=0}^{\infty}\left[\int_{c_{2 k+1}} d T_{L}\right]<\infty .
$$

The last estimate however follows from the same argument employed above in the case where $l_{p}^{+}$was contained in $U$. It suffices to observe that the claim guarantees that $\left|\pi_{z}\left(c_{2(k+1)+1}(0)\right)\right| \leq\left|\pi_{z}\left(c_{2 k+1}(1)\right)\right|$ where $\pi_{z}$ denotes the projection in the coordinate $z$. The theorem is proved.

We can now prove Theorem A'.

Proof of Theorem A'. Consider again a fixed point $p$ and let $\Phi_{p}: \mathbb{C} \rightarrow L_{p}$ be given by $\Phi_{p}(T)=\Phi(T, p)$ where $L_{p}$ stands for the leaf of $\mathcal{D}$ through $p$. In the affine coordinates $\left(x_{1}, \ldots, x_{n-1}, z\right)$, the map $\Phi_{p}$ becomes $\left(\Phi_{1}(T), \ldots, \Phi_{n}(T)\right)$. In particular, this allows us to define the Abelian form $\eta$ on $\mathbb{C}$ by setting $\eta=-\Phi_{n}^{\prime} d T / \Phi_{n}$. Next, if the oriented foliation $\mathcal{H}\left(=\mathcal{H}_{\mathcal{D}}\right)$ is restricted to $L_{p}$, we can consider the corresponding pulled-back oriented foliation $\Phi_{p}^{*} \mathcal{H}$ on $\mathbb{C}$.

Claim 1: The oriented foliation $\Phi_{p}^{*} \mathcal{H}$ coincides with the real (positive) foliation induced by $\eta$.

Proof of Claim 1. Consider a point $q=\Phi_{p}\left(T_{0}\right) \in L_{p}$ that is regular for $\mathcal{H}$. The direction of $\mathcal{H}$ at $q$ is determined by the negative of the gradient of the "height" function $\left(x_{1}, \ldots, x_{n-1}, z\right) \mapsto\|z\|$ restricted to $L_{p}$. In the coordinate $T$ this function is simply $T \mapsto\left\|\Phi_{n}(T)\right\|$. The gradient direction of this function is determined by the condition that $\Phi_{n}^{\prime}\left(T_{0}\right)\left(T-T_{0}\right)$ must be aligned with $\Phi_{n}\left(T_{0}\right)$. This amounts to saying that the direction of $\Phi_{p}^{*} \mathcal{H}$ at $T_{0}$ is nothing but the positive real direction of $\eta$. 
To simplify notation the foliation $\Phi_{p}^{*} \mathcal{H}$ will be denoted by $\{\operatorname{Arg} \eta=0\}$. More generally, the pullback by $\Phi_{p}$ of the foliations $\mathcal{H}^{\theta}$ coincide with $\{\operatorname{Arg} \eta=\theta\}$, in particular $\{\operatorname{Arg} \eta=\pi / 2\}$ is the foliation orthogonal to $\{\operatorname{Arg} \eta=0\}=\Phi_{p}^{*} \mathcal{H}$.

The separating curve $c_{0}$ to be chosen is given by the trajectory of $\{\operatorname{Arg} \eta=\pi / 2\}$ through $T_{0}$ i.e., a trajectory of $\mathcal{H}^{\perp}$. Geometrically, $\Phi_{p}\left(c_{0}\right)$ is the curve determined in $L_{p}$ by the intersection of $L_{p}$ itself with the real hypersurface $\left\{\|z\|=\left|\Phi_{n}\left(T_{0}\right)\right|\right\}$. This curve may be closed. Next, we choose the component $\mathcal{U}^{+}$of $\mathbb{C} \backslash c_{0}$ that corresponds to the saturate of $T_{0}$ by the spray of trajectories of $\{\operatorname{Arg} \eta=\theta\}$ issuing from $T_{0}$ with $\theta \in(-\pi / 2, \pi / 2)$. To check that $\mathcal{U}^{+}$is unbounded just notice that a trajectory $l_{p}^{+, \theta} \subset L_{p}, \theta \in(-\pi / 2, \pi / 2)$, issuing from $p$ will, by construction, accumulate on $\Delta_{\infty}$ unless it accumulates at a singularity of $X$ lying in $\mathbb{C}^{n}$. The statement being clear in the second case, we consider the case where $l_{p}$ accumulates on $\Delta_{\infty}$ so that it leaves every compact set contained in $L_{p}$. Since $X$ is complete, there follows that the integral of the corresponding time-form along $l_{p}^{+, \theta}$ is unbounded. Next, note that the preimage of $l_{p}^{+, \theta}$ under $\Phi_{p}$ is the trajectory of $\{\operatorname{Arg} \eta=\theta\}$ issuing from $T_{0}$. Furthermore, the preimage under $\Phi_{p}$ of the time-form induced on $L_{p}$ is nothing but the canonical form $d T$ on $\mathbb{C}$. Thus the integral of the time-form along a segment of $l_{p}^{+, \theta}$ is equal to the integral of the form $d T$ along the corresponding segment of the trajectory in question. It then follows that this trajectory must leave every compact set contained in $\mathbb{C}$. This shows that $\mathcal{U}^{+}$is unbounded.

In summary, to show that $\mathcal{U}^{+}$satisfies all the conditions in the statement of the theorem, there only remains to check that

$$
\lim _{r \rightarrow \infty} \frac{\operatorname{Meas}\left(\mathcal{T}_{V} \cap B_{r}\right)}{\operatorname{Meas}\left(\mathcal{U}^{+} \cap B_{r}\right)}=1
$$

To begin with, note that $\eta$ is holomorphic in $\mathcal{U}^{+}$since $\Phi_{n}(T)$ never reaches $0 \in \mathbb{C}$. Furthermore the trajectories of $\mathcal{H}^{\theta}, \theta \in(-\pi / 2, \pi / 2)$, approach $\{z=0\}$. These trajectories, in fact, remain in a compact part of the domain of definition of the coordinates $\left(x_{1}, \ldots, x_{n-1}, z\right)$ since the infinity of $\{z=0\}$ consists of poles with residue equal to 1 for the Abelian form $\omega_{1}$ in Section 3; see Lemma 3.5. Hence the same thing happens for $\omega_{1, \mathcal{D}}$ since these forms coincide on $\Delta_{\infty}$. In other words, on a neighborhood of $\Delta_{\infty}^{1, \ldots, n-1}$, the trajectories of $\{\operatorname{Arg} \eta=\pi / 2\}$ are closed curves while the trajectories of $\mathcal{H}^{\theta}$ for $\theta \in(-\pi / 2, \pi / 2)$ point outward along these closed curves. The preceding claim is then clear. As a consequence of this, we conclude that the absolute value of the coefficient of $\eta$ is uniformly bounded in $\mathcal{U}^{+} \backslash \mathcal{T}_{V}$ since away from $\mathcal{T}_{V}$ the form $\omega_{1, \mathcal{D}}$ is bounded from below by a positive constant. As already explained in Remark 6.3, $\eta$ defines a singular flat structure on $\mathcal{U}^{+}$for which the trajectories of $\{\operatorname{Arg} \eta=\theta\}$ are geodesics. This leads us to:

Claim 2: Given $\varepsilon>0$, there is $\delta>0$ such that the saturate $\mathcal{U}_{\delta}^{+}$of $T_{0}$ by trajectories of $\{\operatorname{Arg} \eta=\theta\}$ for $\theta \in(-\pi / 2+\delta, \pi / 2-\delta)$ verifies

$$
\liminf _{r \rightarrow \infty} \frac{\operatorname{Meas}\left[\left(\mathcal{U}_{\delta}^{+} \cap B_{r}\right) \cup\left(\mathcal{T}_{V} \cap\left(\mathcal{U}^{+} \backslash \mathcal{U}_{\delta}^{+}\right)\right)\right]}{\operatorname{Meas}\left(\mathcal{U}^{+} \cap B_{r}\right)}>1-\varepsilon .
$$


Proof of Claim 2. The statement would be clear if the flat structure induced by $\eta$ were the standard flat structure of $\mathbb{C}$. More generally, suppose that $\eta$ has no singular points and consider an arc of circle $S_{r_{0}}$ (centered at $T_{0}$ ) of radius $r_{0}$ whose interior contains no singular points of $\eta$. Consider also two trajectories $l_{\theta_{1}}$ and $l_{\theta_{2}}$ issuing from $T_{0}$. Since $X$ is complete these trajectories intersect $S_{r_{0}}$ at points $T_{1}$ and $T_{2}$. Now consider the triangle whose sides are the segments of $l_{\theta_{1}}$ and $l_{\theta_{2}}$ delimited by $T_{0}$ and by $T_{1}$ and $T_{2}$ together with the corresponding arc of $S_{r_{0}}$ determined by $T_{1}$ and $T_{2}$. Because $\eta$ is closed, the integral of $\eta$ along the boundary of this triangle equals zero. Finally, since the coefficient of $\eta$ is uniformly bounded (and bounded from below if we avoid a fixed small neighborhood of its singular points), we conclude that the length of the arc of $S_{r_{0}}$ determined by $T_{1}, T_{2}$ is bounded by $C r_{0}\left|\theta_{1}-\theta_{2}\right|$ for every pair $\theta_{1}$ and $\theta_{2}$ and for some constant $C$. The desired estimate follows from this since $S_{r_{0}}$ contains no singularities of $\eta$ in its interior.

To finish the proof of the claim, we need to consider the effect of the singularities of $\eta$. These singularities are of saddle type since $\eta$ is holomorphic on $\mathcal{U}^{+}$. For every singular point of $\eta$, we consider a disc of uniform radius around the corresponding point in $L_{p}$. In the complement of the union of these discs, the form $\omega_{1, \mathcal{D}}$, and hence $\eta$ in the coordinate $T$, is bounded from below by a positive constant. The claim will be proved if the union of these discs in the coordinate $T$ has area less than $\varepsilon r / 2$ for $r$ large. In fact, in the complement of this union $\eta$ is bounded from below by a positive constant and from above by the previous constant so that the preceding argument can be applied in finitely many regions of a ball $B_{r}$. Finally, to justify the previous claim note that, in order to prove the desired estimate, we only need to consider those discs centered at points in $L_{p}$ that lie in the complement of $V$. Therefore the norm of $X$ is bounded from below by a positive constant in these discs which, in turn, ensures that their size in the coordinate $T$ is uniformly bounded. Furthermore, the distance in the leaf $L_{p}$ between every two discs as before is bounded from below by a positive constant. Though this property is not directly reflected in the coordinate $T$ since the norm of $X$ increases (i.e., $X$ becomes faster), the size of the corresponding neighborhoods reduces in the same proportion as the norm of $X$ increases. This quickly leads to the desired conclusion and establishes the claim.

In view of Claim 2 to finish the proof of Theorem A' it suffices to show that

$$
\lim _{r \rightarrow \infty} \frac{\operatorname{Meas}\left(\mathcal{T}_{V} \cap B_{r} \cap \mathcal{U}_{\delta}^{+}\right)}{\operatorname{Meas}\left(\mathcal{U}_{\delta}^{+} \cap B_{r}\right)}=1,
$$

for fixed positive $\delta$. To do this, consider $r$ given. Next, note that every for $\theta \in$ $(-\pi / 2+\delta, \pi / 2-\delta)$ the corresponding trajectory $l_{\theta}$ of $\{\operatorname{Arg} \eta=\theta\}$ issuing from $T_{0}$ intersects the boundary $\partial B_{r}$ of $B_{r}$ since $X$ is complete. Let $T_{\theta, r}$ be this intersection point and denote by $l_{\theta, r}$ the segment of $l_{\theta}$ delimited by $T_{0}$ and $T_{\theta, r}$. According to Theorem A, there is uniform constant Cte (depending neither on $\theta$ nor on $r$ ) such that the length of the segments of $l_{\theta, r}$ corresponding to those instants where $\Phi(T)$ remains away from $V$ is bounded by Cte whereas the length of $l_{\theta, r}$ goes to infinity as $r \rightarrow \infty$. The conclusion of Theorem A' now results from a standard application of Fubini's theorem. 


\subsection{Complete polynomial vector fields on $\mathbb{C}^{n}$ with simple singularities at infinity}

In this section we give an application of Theorem 5.1 that cannot be obtained from Theorem 4.7 alone. Let $X$ be a complete polynomial vector field on $\mathbb{C}^{n}$ and denote by $\mathcal{D}$ its associated foliation. Recall that we make no distinction between $\mathcal{D}$ viewed as a foliation of $\mathbb{C}^{n}$ and $\mathcal{D}$ viewed as a foliation of $\mathbb{C P}^{n}$. Again $X_{d}$ denotes the homogeneous component of $X$ of highest degree where $d$ stands for this degree and $d \geq 2$. The foliation associated with $X_{d}$ is denoted by $\mathcal{F}$ and can also be viewed as a foliation of either $\mathbb{C}^{n}$ or $\mathbb{C P}^{n}$. Recall that the singularities of $\mathcal{D}$ in the hyperplane at infinity $\Delta_{\infty}$ are assumed to be simple in the sense of Conditions (1) and (2) given in the introduction, just before the statement of Theorem B. It follows that these singularities are isolated in $\Delta_{\infty}$ (but maybe not in $\mathbb{C P}^{n}$ ). Furthermore, if $X_{d}$ is divisible by a nonconstant polynomial $P$, then $P$ must also divide $X$. Otherwise, the curve induced on $\Delta_{\infty}$ by $\{P=0\}$ would contain singularities of $\mathcal{D}$ whose linear part is degenerate; this is impossible since these singularities are simple by assumption. In turn, the last observation implies that the singular sets in $\Delta_{\infty}$ of $\mathcal{D}$ and of $\mathcal{F}$ coincide. Finally, $X_{d}$ must have a singular set of codimension at least 2 since the singular set of $X$ has codimension at least 2 .

The reader is reminded that the restriction of $\mathcal{D}$ to $\Delta_{\infty}$ coincides with the foliation $\mathcal{F}_{\infty}$ induced by $\mathcal{F}$ on $\Delta_{\infty}$. Moreover, if $q \in \Delta_{\infty}$ is a (necessarily common) singular point of $\mathcal{D}$ and $\mathcal{F}$, then the corresponding linear parts of these foliations at $q$ coincide as well.

Lemma 6.5. With the definition of leaf from Section 2 , the leaves of $\mathcal{F}_{\infty}$ are either rational curves or quotients of $\mathbb{C}$.

Proof. We need to show that the leaves of $\mathcal{F}_{\infty}$ cannot be hyperbolic Riemann surfaces. Since $\mathbb{C P}^{n}$ has a Kähler structure, it follows from the main result of [6] that the set of parabolic leaves of $\mathcal{D}$ is a pluripolar set unless it coincides with the whole space. Since the leaves of $\mathcal{D}$ contained in $\mathbb{C}^{n}$ are clearly parabolic, we conclude that the leaves of $\mathcal{D}$ contained in $\Delta_{\infty}$ must also be parabolic (or rational). These leaves, however, are nothing but the leaves of $\mathcal{F}_{\infty}$.

The next step is to consider the foliation $\widetilde{\mathcal{F}}$ induced on the manifold $M$ by $\mathcal{F}$. In particular, the foliation $\widetilde{\mathcal{F}}_{\infty}$ is naturally identified with $\mathcal{F}_{\infty}$. We return to using the notation of Section 5 and denote by $\widetilde{X}_{d}$ the transform of $X_{d}$ on $M$. In view of the existence of the projections $\mathcal{P}_{0}$ and $\mathcal{P}_{\infty}$ introduced in Section 3, Lemma 6.5 implies that no leaf of $\widetilde{\mathcal{F}}$ is hyperbolic. Note that this conclusion cannot be derived directly from the vector field $X_{d}$ since $X_{d}$ need not be complete (it is only semicomplete).

The next proposition relies heavily on Theorem 5.1 and it will play a crucial role in the proof of Theorem B.

Proposition 6.6. Assuming $d \geq 2$, there exists a singularity of $\widetilde{\mathcal{F}}_{\infty}$ providing a sink singularity for $\mathcal{H}$ (resp. $\left.\mathcal{H}^{\theta}\right)$ restricted to a generic leaf of $\widetilde{\mathcal{F}}_{\infty}$. 
In the sequel, we shall prove Theorem B assuming that Proposition 6.6 holds. To this end, Proposition 6.6 can be summarized by saying that there is a singularity $q \in \Delta_{\infty}$ of $\widetilde{\mathcal{F}}$ all of whose eigenvalues $\lambda_{1}^{q}, \ldots, \lambda_{n}^{q}$ belong to $\mathbb{R}_{+}^{*}$. This assertion, in turn, can slightly be improved. Indeed, consider the vector field $X_{d}$ and local coordinates as in Section 3, where $q \simeq 0$ and $\left\{x_{n}=0\right\}$ is contained in the hyperplane at infinity of $M$ (and hence identified with the coordinate $z$ used in Section 3). In these local coordinates, the vector field $\widetilde{X}_{d}$ has the form

$$
\widetilde{X}_{d}=Q Y_{1}+\text { h.o.t }
$$

where $Q$ is a rational function and $Y_{1}$ is a linear vector field with real positive eigenvalues $\lambda_{1}^{q}, \ldots, \lambda_{n}^{q}$. Since we have seen that $X^{d}$ has a singular set of codimension at least $2, Q$ is a rational function having a (unique) polar component of degree $d-1$ passing through $q$ (and coinciding with $\Delta_{\infty}$ ) and empty zero divisor. Thus the foliation associated to $Q Y_{1}$ must have a smooth separatrix transverse to $\Delta_{\infty}$. Upon restricting $Q Y_{1}$ to this separatrix the semi-complete condition implies that $d$ must be equal to 2 . Furthermore, the local holonomy of the separatrix in question must be trivial (see, for example, [23]). Therefore each of the eigenvalues $\lambda_{1}^{q}, \ldots, \lambda_{n-1}^{q}$ is a multiple of the eigenvalue $\lambda_{n}^{q}$. Hence we can set $\lambda_{n}^{q}=1$ and $\lambda_{1}^{q}, \ldots, \lambda_{n-1}^{q} \in \mathbb{Z}_{+}$. This refined statement leads us to:

Lemma 6.7. The set formed by the separatrices of $\mathcal{D}$ at $q$ contains nontrivial open sets of $\mathbb{C}^{n}$.

Proof. Consider the vector field $X$ (resp. foliation $\mathcal{D}$ ) on a neighborhood of the singularity $q \in \Delta_{\infty}$. The reader is reminded that the foliations $\mathcal{D}$ and $\mathcal{F}$ have the same singularities in $\Delta_{\infty}$ and each of these (common) singularities have the same eigenvalues. Thus what precedes implies that $\mathcal{D}$ has $n$ eigenvalues $\lambda_{1}^{q}, \ldots, \lambda_{n}^{q}$ at $q$ and these eigenvalues are strictly positive integers. Therefore they belong to the Poincaré domain so that the corresponding local vector field is either linearizable or it admits a Poincaré-Dulac normal form. Since the latter possibility was ruled out by assumption, our local vector field must be linearizable at $q$. The statement follows now from the fact that $\left\{\lambda_{1}^{q}, \ldots, \lambda_{n}^{q}\right\} \subset \mathbb{Z}_{+}$.

Proof of Theorem B. Lemma 6.7 ensures the existence of a singular point $q \in \Delta_{\infty}$, where all the eigenvalues $\lambda_{1}^{q}, \ldots, \lambda_{n}^{q}$ of $\mathcal{D}$ belong to $Z_{+}^{*}$. Furthermore $\mathcal{D}$ is locally linearizable about $q$. Consider now a generic leaf $L$ of $\mathcal{D}$ defining a (local) separatrix $\mathcal{S}$ for $\mathcal{D}$ at $q$. Having fixed $\mathcal{S}$, consider also a local irreducible Puiseux parametrization $\gamma(t)$ for $\mathcal{S}$ defined on a neighborhood of $0 \in \mathbb{C}$ and satisfying $\gamma(0)=q$. Next, in suitable local coordinates $\left(u_{1}, \ldots, u_{n-1}, z\right)$ centered at $q$, with $\{z=0\} \subset \Delta_{\infty}$, the vector field $X$ has the form $f\left(u_{1}, \ldots, u_{n-1}, z\right) . Y$ where $Y$ is a linear vector field with eigenvalues $\lambda_{1}^{q}, \ldots, \lambda_{n}^{q}$ and $f$ is a meromorphic function whose zero divisor is empty and whose pole divisor is contained in $\{z=0\} \subset \Delta_{\infty}$.

Consider the restriction $X_{\mathcal{S}}$ of $X$ to $\mathcal{S}$ pulled-back by $\gamma$. With respect to the coordinate $t$ the vector field $\gamma^{*} X_{\mathcal{S}}$ has the form

$$
\gamma^{*} X_{\mathcal{S}}=f \circ \gamma(t)\left(\gamma^{*} Y_{\mathcal{S}}\right)
$$


where $\gamma^{*} Y_{\mathcal{S}}$ stands for the pullback by $\gamma$ of the restriction of $Y$ to $\mathcal{S}$. It is immediate to check that the order of the one-dimensional vector field $\gamma^{*} Y_{\mathcal{S}}$ at $t=0$ equals 1 . Thus the order of $\gamma^{*} X_{\mathcal{S}}$ at $t=0$ is simply $1+\operatorname{ord}_{t=0}(f \circ \gamma(t))$ where $\operatorname{ord}_{t=0}(f \circ \gamma(t))$ is the order at $t=0$ of the function $t \mapsto f \circ \gamma(t)$. Since the zero divisor of $f$ is empty, the order of this function is strictly negative provided that $X$ has, in fact, poles on $\Delta_{\infty}$.

To finish the proof of Theorem B we proceed as follows. Since the restriction of $X$ to $X_{\mathcal{S}}$ is semi-complete, the order at $t=0$ of the vector field $\gamma^{*} X_{\mathcal{S}}$ belongs to the set $\{0,1,2\}$; see Section 2.2. However, if the degree of the vector field $X$ exceeds 1 , then $X$ does have poles on $\Delta_{\infty}$. Hence the above discussion implies that the order of $\gamma^{*} X_{\mathcal{S}}$ at $t=0$ must be zero, i.e., $\gamma^{*} X_{\mathcal{S}}$ is regular at $t=0$. Therefore the flow of $X_{\mathcal{S}}$ reaches the point $t=0 \simeq q \in \Delta_{\infty}$ in finite time. This is however impossible since $X$ is semi-complete. The resulting contradiction shows that the degree of $X$ is bounded by 1 and the statement of the theorem follows.

Let us close this section with the proof of Proposition 6.6. The argument goes by contradiction so that we assume from now on that no singularity of $\widetilde{\mathcal{F}}_{\infty}$ yields a sink singularity for $\mathcal{H}$ (resp. $\mathcal{H}^{\theta}$ ) restricted to a generic leaf of $\widetilde{\mathcal{F}}_{\infty}$. A rather direct way to obtain a contradiction out of this assumption is as follows. Recall that in Section 6.1, Theorem 4.7 was adapted to apply to the (nonhomogeneous) vector field $X$, along with its associated foliation $\mathcal{D}$. The same adaptation can straightforwardly be made for Theorem 5.1. Thus, assuming that the trajectories of $\mathcal{H}$ have no future endpoints, we conclude that the flow of $X$ is not defined at a certain point $t_{0} \in \mathbb{C}$. This is clearly impossible since $X$ is complete.

We shall, however, provide a less direct argument that will also enable us to quickly derive Theorem C. Namely, by building on the semi-complete character of the (homogeneous) vector field $X_{d}$, we are going to show that the leaves of the foliation $\mathcal{F}_{\infty}$ are hyperbolic Riemann surfaces provided that no singular point of $\widetilde{\mathcal{F}}_{\infty}$ yields a sink singularity for $\mathcal{H}$ restricted to a generic leaf of $\widetilde{\mathcal{F}}_{\infty}$. The statement of Proposition 6.6 will then follow from Lemma 6.5.

Proof of Proposition 6.6. In what follows we assume $d \geq 2$. Aiming at a contradiction, we suppose that no singular point of $\widetilde{\mathcal{F}}_{\infty}$ satisfies the condition in the statement of the proposition. In view of the material in Section 5, and given that the singularities of $\mathcal{D}$ are isolated inside $\Delta_{\infty}$, we conclude that a trajectory of $\mathcal{H}$ has no future endpoint once it is contained in a generic leaf of $\mathcal{D}$. In particular, all the corresponding trajectories have infinite length. This condition will be exploited in the sequel.

Recall that $\widetilde{X}_{d}$ stands for the transform of $X_{d}$ on $M$ where $\widetilde{\mathcal{F}}$ denotes the foliation associated with $\widetilde{X}_{d}$. As mentioned, our strategy for obtaining a contradiction consists of showing that a generic leaf of $\widetilde{\mathcal{F}}_{\infty}$ cannot be a parabolic Riemann surface. For this, consider a leaf $L_{\infty} \subseteq \Delta_{\infty}$ and points $p \in L_{\infty}$ and $q \in \mathcal{P}_{\infty}^{-1}(p)$ as in the statement of Theorem 5.1. Denote by $L_{q}$ the leaf of $\widetilde{\mathcal{F}}$ through $q$ so that $\mathcal{P}_{\infty}\left(L_{q}\right)=L_{\infty}$. Note that the leaf $L_{q}$ can be assumed to be a generic leaf of $\widetilde{\mathcal{F}}$ in the above sense. The vector field $\widetilde{X}_{d}$ is regular at a generic point of $L_{q}$. Indeed, since $L_{q}$ is a parabolic Riemann surface, $\widetilde{X}_{d}$ is holomorphic on $L_{q}$ and contains 
at most two zeros as follows from the classification of semi-complete vector fields on parabolic Riemann surfaces; see Section 2.2. The set of possible zeros for the restriction of $X$ to $L_{q}$ will be denoted by $\left\{X_{\mid L_{q}=0}\right\}$. Next consider the maximal domain of definition $\mathbf{U} \subseteq \mathbb{C}$ where the semi-global flow $\phi$ associated to $\widetilde{X}_{d}$ with initial condition $\phi(0)=q$ is defined. The map $\phi$ yields a covering map from $\mathbf{U}$ to $L_{q} \backslash\left\{X_{\mid L_{q}=0}\right\}$.

The structure of the set $\left\{X_{\mid L_{q}=0}\right\}$ can be described more accurately. In fact, since $\widetilde{X}_{d}$ is homogeneous, a point in $\left\{X_{\mid L_{q}=0}\right\}$ must correspond to a singular point of $\widetilde{\mathcal{F}}$ that was added to $L_{q}$ due to our definition of leaf in Section 2.2. In particular, $L_{q}$ defines a (local) separatrix for $\widetilde{\mathcal{F}}$ at some of its singular points. Next note that the singularity in question can be supposed to lie away from $\Delta_{\infty}$. In fact, since we are assuming that our proposition is not verified, none of the singularities of $\widetilde{\mathcal{F}}_{\infty}$ in $\Delta_{\infty}$ is dicritical. Thus, the saturate of the set formed by all their local separatrices has empty interior. Hence it suffices to choose $L_{q}$ sufficiently generic to ensure it does not define a local separatrix for any of the singularities of $\widetilde{\mathcal{F}}_{\infty}$ in $\Delta_{\infty}$. It follows that the singular point in question lies in the affine $\mathbb{C}^{n}$. Since $d \geq 2$ and $\widetilde{X}_{d}$ comes from a homogeneous (degree $d$ ) vector field, there also follows that the order of $X$ at a point in $\left\{X_{\mid L_{q}=0}\right\}$ must be strictly greater than 1 . Therefore it must be equal to 2 .

The preceding discussion shows that the order of every zero of $X_{\mid L_{q}=0}$ equals 2 . Considering again the covering map $\phi: \mathbf{U} \rightarrow L_{q} \backslash\left\{X_{\mid L_{q}=0}\right\}$, it follows that every point in $\left\{X_{\mid L_{q}=0}\right\}$ induces in the coordinate $t$ a neighborhood of $t=\infty \in \mathbb{C} \cup\{\infty\}$. Since the flow is semi-complete, it follows that the set $\left\{X_{\mid L_{q}=0}\right\}$ can actually contain at most a single point of $L_{q}$. Therefore, when $\left\{X_{\mid L_{q}=0}\right\}$ is not empty, it is natural to consider the map $\phi$ extended to $\mathbf{U} \cup\{\infty\}$ where $\phi(\infty)$ is the (unique) zero of $X_{\mid L_{q}=0}$.

We return to $\mathbf{U} \subset \mathbb{C}$. Owing to Theorem 5.1, the integral of the corresponding time-form over the $\mathcal{H}$-trajectory $l_{q}$ converges. This convergence implies that the lift of $l_{q}$ in $\mathbf{U}$ has finite length for the Euclidean metric on $\mathbb{C}$ since the length in question is nothing but the mentioned integral. We conclude that the lift of $l_{q}$ to $\mathbf{U}$ converges to a point $t_{0}$ in the boundary of $\mathbf{U}$.

Note that $L_{q}$ cannot be contained in either a rational or elliptic curve, otherwise it would induce a separatrix for some singular point in of $\widetilde{\mathcal{F}}$ in $\Delta_{\infty}$. Thus $L_{q}$ is not contained in any compact Riemann surface contained in $M$. Suppose now that $L_{q}$ is a parabolic Riemann surface. It then follows that either $\mathbf{U}=\mathbb{C} \backslash\left\{t_{0}\right\}$ or $\mathbf{U}=\mathbb{C} \backslash\left\{t_{0}, t_{1}\right\}$ where, in the latter case, the point $t=\infty$ must belong to $L_{q}$. Now, let the plane $\mathbb{C}$ be equipped with the (time) coordinate $t$ so that the canonical form $d t$ coincides with the pullback by $\phi$ of the time-form induced on $L_{q}$ by $X$. Also, denote by $\mathcal{H}_{t}$ the lift of the foliation $\mathcal{H}$ (restricted to $L_{q}$ ) to the $\mathbb{C}$-plane. The foliation $\mathcal{H}_{t}$ is naturally a foliation defined on $\mathbf{U}$ which, in turn, is isomorphic to either $\mathbb{C} \backslash\left\{t_{0}\right\}$ or to $\mathbb{C} \backslash\left\{t_{0}, t_{1}\right\}$ where $t_{1} \in \mathbb{C}$ is some point distinct from $t_{0}$.

Consider the case $\mathbf{U}=\mathbb{C} \backslash\left\{t_{0}\right\}$. As the reader will observe, the case where $\mathbf{U}=\mathbb{C} \backslash\left\{t_{0}, t_{1}\right\}$ can be treated analogously. First, we need to determine the local behavior of $\mathcal{H}$, or of $\mathcal{H}_{t}$, in a neighborhood of $t_{0}$. For this, recall that $\mathcal{H}_{t}$ 
is the foliation induced on the plane $\mathbb{C}$ by the 1 -form $\phi^{*} \omega_{1}$ which is meromorphic on $\mathbf{U}=\mathbb{C} \backslash\left\{t_{0}\right\}$ but may have an essential singular point at $t_{0}$. In our previous discussion, the behavior of $\mathcal{H}$ on a neighborhood of points that are zeros or poles for the 1-form in question was determined and this led to the singular points of types sink, source and saddle. However, nothing was said about the behavior of $\mathcal{H}$ near an essential singular point for the corresponding 1-form. To deal with this case, we shall need to introduce a new type of singular point for $\mathcal{H}_{t}$ which can appear at an essential singularity of the corresponding 1-form. This type of singular point is going to be called an improper sink for $\mathcal{H}_{t}$ (or for $\mathcal{H}$ ). By definition, a point $T \in \mathbb{C}$ is an improper sink if all the $\mathcal{H}_{t}$-trajectories through points close to $T$ converge to $T$. Thus, in the time coordinate $t$, the topological behavior of $\mathcal{H}_{t}$ near an improper sink is the same as near a sink (in the usual sense). However the reader should also note that, in this improper sink situation, whereas the $\mathcal{H}$-trajectories viewed in the coordinate $t$ appear to converge to the singular point, when these trajectories are viewed as they were defined in the actual leaf $L_{q}$, they turn out to have infinite length.

With the assumption that $\mathbf{U}=\mathbb{C} \backslash\left\{t_{0}\right\}$, we have the following claim.

Claim. The point $t_{0}$ is an improper sink for $\mathcal{H}_{t}$.

Proof of the Claim. Clearly $L_{q}$ has a cylindrical end since it is parametrized by a punctured neighborhood of $t_{0}$ in the $\mathbb{C}$-plane. In other words, the map $\phi$ allows us to realize a punctured neighborhood of $t_{0} \in \mathbb{C}$ as an end of $L_{q}$. Next consider a point $q^{\prime}=\phi\left(t^{\prime}\right)$ for $t^{\prime}$ near $t_{0}$. The trajectory of $\mathcal{H}$ through $q^{\prime}$ is infinite and thus the integral of the corresponding time-form over this trajectory must converge again to a point lying on the boundary of $\mathbf{U}$. We then conclude that the mentioned integral converges to $t_{0}$. In the $\mathbb{C}$-plane equipped with the coordinate $t$, the preceding translates into the fact that the integral of the canonical form $d t$ along the lift (via $\phi$ ) of the $\mathcal{H}$-trajectory through $q^{\prime}$ converges to $t_{0}$. This lift, however, is nothing but the trajectory of $\mathcal{H}_{t}$ through $t^{\prime}$. It then follows that the trajectory in question converges to $t_{0}$ and this is the content of the claim.

The reader will observe that in, the case $\mathbf{U}=\mathbb{C} \backslash\left\{t_{0}, t_{1}\right\}$, all the trajectories mentioned in the above proof must converge either to $t_{0}$ or to $t_{1}$ so that the statement of the claim must be adapted by saying that the set $\left\{t_{0}, t_{1}\right\}$ yields an improper sink singularity for $\mathcal{H}_{t}$.

Summarizing what precedes, the foliation $\mathcal{H}_{t}$ on the plane $\mathbb{C}$ has a unique improper sink singularity, corresponding to $t=t_{0}$, and no (ordinary) sink singularity. Furthermore all trajectories of $\mathcal{H}_{t}$ converge to $t_{0}$. Indeed, the integral of the time-form over every trajectory of $\mathcal{H}$ converges to a point in the boundary of $\mathbf{U}=\mathbb{C} \backslash\left\{t_{0}\right\}$ and hence to $t_{0}$ itself.

Here is a good place to observe that the preceding conclusions still have natural analogues in the case where $\mathbf{U}=\mathbb{C} \backslash\left\{t_{0}, t_{1}\right\}$. Indeed, the preceding discussion ensures that all trajectories of $\mathcal{H}_{t}$ will converge to either $t_{0}$ or to $t_{1}$.

To finish the proof of the proposition, we are going to show that the situation described above cannot happen. In fact, let $t_{1}^{*}$ be a source singularity of $\mathcal{H}_{t}$. Note that $t_{1}^{*}$ exists since this of type singularity is produced by the intersections 
of $\mathcal{P}_{\infty}\left(L_{q}\right)$ with the hyperplane at infinity $\Delta_{\infty}^{(x, y)}$ in the coordinates $(x, y)$ (i.e., the hyperplane at infinity corresponding to affine coordinates for $\left.\Delta_{\infty}\right)$. Since $\Delta_{\infty}^{(x, y)}$ neither is invariant under $\widetilde{\mathcal{F}}_{\infty}$ nor are there singularities of $\widetilde{\mathcal{F}}_{\infty}$ lying in $\Delta_{\infty}^{(x, y)}$, it follows that every leaf of $\widetilde{\mathcal{F}}_{\infty}$ intersects $\Delta_{\infty}^{(x, y)}$ nontrivially. Furthermore these intersections then produce source singularities for $\mathcal{H}_{t}$. These source singularities also have residue equal to 1 .

Next, note that away from saddle-singularities of $\mathcal{H}_{t}$, this foliation can be given a structure of a transverse Riemannian foliation; just parameterize the trajectories of the orthogonal foliation by the integral of $\phi^{*} \omega_{1}$. Thus there is a region $I_{1}^{*}$ on a small loop $c$ around $t_{0}$ such that the integral of $\phi^{*} \omega_{1}$ over $I_{1}^{*}$ equals -1 (the negative of the residue of $\phi^{*} \omega_{1}$ at $\left.t_{1}^{*}\right)$. In fact, $I_{1}^{*}$ is obtained by the intersections with $c$ of the trajectories emanating from $t_{1}^{*}$ (recall that they all converge to $t_{0}$ ). However, $c$ can be chosen so that $\phi^{*} \omega_{1}$ is bounded on a neighborhood of $c$ and hence the integral of $\phi^{*} \omega_{1}$ over $c$ is well defined. To derive a final contradiction, it suffices to observe that in the complement of the small disc bounded by $c$, there are infinitely many source singularities $t_{1}^{*}, t_{2}^{*}, \ldots$ for $\mathcal{H}_{t}$. In fact, in the above construction, each of these singularities determines as above a region $I_{k}^{*}$ over which the integral of $\phi^{*} \omega_{1}$ equals -1 . Finally all these regions $I_{1}^{*}, I_{2}^{*}, \cdots$ are clearly pairwise disjoint which immediately leads to a contradiction with the existence of a bound for $\phi^{*} \omega_{1}$ on a neighborhood of $c$.

Once again we note that $c$ must be thought of as the union of two curves bounding two (small) discs around $t_{0}$ and $t_{1}$ when we have the case $\mathbf{U}=\mathbb{C} \backslash\left\{t_{0}, t_{1}\right\}$.

Finally, there remains only to check the existence of infinitely many source singularities $t_{1}^{*}, t_{2}^{*}, \ldots$ in the complement of the disc bounded by $c$ (and containing $t_{0}$ ). For this, note first that $t_{0}$ must be an essential singularity of $\phi$ so that the image under $\phi$ of a small neighborhood of $t_{0}$, viewed in $(x, y)$ coordinates, cannot be bounded. Thus the image of this neighborhood must accumulate on $\Delta_{\infty}^{(x, y)}$. However, since $\widetilde{\mathcal{F}}_{\infty}$ is transverse to $\Delta_{\infty}^{(x, y)}$, whenever this image gets close enough to $\Delta_{\infty}^{(x, y)}$, it is forced to intersect this curve. Therefore we can assume that in the complement of the curve $c$ there are already a certain number (at least 3) of source singularities for $\mathcal{H}_{t}$. A similar argument applies for $t=\infty$. Thus, if in the complement of $c$ there are only finitely many source singularities, then $\phi(t)$ must be bounded on a neighborhood of $t=\infty$. This case actually means that $t=\infty$ is a singularity of $\widetilde{\mathcal{F}}$ where the vector field $X_{\mid L_{q}=0}$ has a zero of order 2 .

To obtain a contradiction, we proceed as follows. Consider $T \in \mathbb{C}$ with $|T|$ very large. The semi-global flow $\phi^{T}$ induces an automorphism of $L_{q}$ in a sense adapted to the domain of definition of this semi-global flow. Because there are already (more than 3) points in this domain whose image by $\phi$ lies in $\Delta_{\infty}$, there must also exist $q^{\prime} \in L_{q}$ such that $\phi^{T}\left(q^{\prime}\right)$ is well defined and lies in $\Delta_{\infty}^{(x, y)}$. To conclude the statement, we just need to show that arbitrarily close to $t_{0}$, there is a point $\tilde{t} \neq t_{0}$ such that $\phi^{\tilde{t}}(q)$ is close to $q^{\prime}$. In fact, in this case $\phi(T+\tilde{t})$ is close to $\Delta_{\infty}^{(x, y)}$. The reader will note that $\phi(T+\tilde{t})$ is well defined for $|T|$ very large since $\phi$ is defined on a neighborhood of $t=\infty$. Finally the existence of $\tilde{t}$ is an immediate application of the elementary Casorati-Weierstrass theorem. The proposition is proved. 
Remark 6.8. Throughout this paper, the affine coordinates $(x, y, z)$ (as well as their higher dimensional versions) used in the construction of $M$ where chosen generic in the sense that $\Delta_{\infty}^{(x, y)}$ remained away from the singularities of $\widetilde{\mathcal{F}}_{\infty}$. In particular, in the context of Theorem B, this was useful to establish Proposition 6.6 guaranteeing the existence of a singular point all of whose eigenvalues are strictly positive. This argument will be repeated in the context of Theorem $\mathrm{C}$ to be proved in Section 7.1. The point we want to make here is that further information, such as the existence of a second singularity with a similar property, may be obtained by choosing the coordinates $(x, y, z)$ so as to make $\Delta_{\infty}^{(x, y)}$ pass through the first singularity having only strictly positive eigenvalues. In other words, specific (i.e., nongeneric) choices of coordinates may also yield useful information.

The choice of coordinates where $\Delta_{\infty}^{(x, y)}$ contains a singular point all of whose eigenvalues are strictly positive is particularly useful because these singular points have a tendency to produce sink singularities for $\mathcal{H}$. This is however compensated by the tendency of $\Delta_{\infty}^{(x, y)}$ to produce source singularities. In fact, under rather general conditions, this singular point lying in $\Delta_{\infty}^{(x, y)}$ no longer yields future endpoints for the trajectories of $\mathcal{H}$. In particular, if the leaves of $\widetilde{\mathcal{F}}_{\infty}$ are known to be parabolic Riemann surfaces, the trajectories of $\mathcal{H}$ must have a future endpoint and this endpoint cannot be the initial singular point belonging to $\Delta_{\infty}^{(x, y)}$. Hence there must exist another singular point of $\widetilde{\mathcal{F}}_{\infty}$ yielding a sink singularity for $\mathcal{H}$ and this establishes our claim on the existence of another singularity of $\widetilde{\mathcal{F}}_{\infty}$ with positive eigenvalues.

\section{Theorem $\mathrm{C}$ and Halphen vector fields}

This section contains the proofs of Theorems C and C' along with new proofs for some results obtained in [26] concerning Halphen vector fields. Since the results given here about Halphen vector fields are not original, our discussion can be regarded simply as a further illustration of our techniques. Nonetheless, the setting introduced here makes sense for much more general vector fields transverse to singular fibrations and conceivably similar ideas can be applied to certain Painlevé equations as well as to other classical equations including several Chazy equations.

\subsection{Painlevé-Guillot's lattices of quadratic vector fields: examples and results}

This paragraph concerns an application of our techniques to the work of A. Guillot in [25] and [26] (see also [23] for an introduction to both papers). We put ourselves in the context of [25]. Hence $X^{2}$ stands for a semi-complete homogeneous quadratic vector field on $\mathbb{C}^{n}$ with isolated singularities. The foliation $\widetilde{\mathcal{F}}$ associated to $X^{2}$ on $M$ is assumed to leave $\Delta_{\infty}\left(\right.$ resp. $\left.\Delta_{0}\right)$ invariant. The pole divisor (resp. zero divisor) of the lift to $M$ of $X^{2}$ consists of $\Delta_{\infty}$ (resp. $\Delta_{0}$ ) with multiplicity 1. Finally, the singularities of $\widetilde{\mathcal{F}}$ in $\Delta_{\infty}$ (resp. $\Delta_{0}$ ) have three eigenvalues different 
from zero since $X^{2}$ has isolated singularities. From this, it also follows that $\widetilde{\mathcal{F}}$ has exactly $2^{n}-1$ singularities on $\Delta_{\infty}\left(\right.$ resp. $\left.\Delta_{0}\right)$.

We denote by $p_{1}, \ldots, p_{2^{n}-1}$ (resp. $q_{1}, \ldots, q_{2^{n}-1}$ ) the singularities of $\widetilde{\mathcal{F}}$ in $\Delta_{0}$ (resp. their dual singularities in $\Delta_{\infty}$ ). Following the convention of [25], the eigenvalues of $\widetilde{\mathcal{F}}$ at $p_{i}$ are $1, u_{1}^{i}, \ldots, u_{n-1}^{i}$ where 1 is the eigenvalue corresponding to the radial direction. According to Guillot, for a semi-complete vector field $X^{2}$ as above, $u_{1}^{i}, \ldots, u_{n}^{i}$ are all integers. Moreover, by setting $\xi_{i}=u_{1}^{i} \times \cdots \times u_{n-1}^{i}$, the vector field $X^{2}$ yields a solution for the equation

$$
\sum_{i=1}^{2^{n}-1} \frac{1}{\xi_{i}}=(-1)^{n+1}
$$

Thus the problem of classifying vector fields as above is related to the problem of finding all these Egyptian fractions. This problem is however quite intricate as is attested by the multitude of interesting examples presented in the above mentioned works. Our contribution to it begins with the following lemma:

Lemma 7.1. The leaves of a vector field in the Painlevé-Guillot lattice having no dicritical singularity at infinity are hyperbolic Riemann surfaces.

Proof. If there is no dicritical singularity in $\Delta_{\infty}$, there cannot exist a future endpoint for the trajectories of $\mathcal{H}$ and $\mathcal{H}^{\theta}, \theta \in(-\pi / 2, \pi / 2)$ for a generic leaf. In other words, the trajectories of the foliations $\mathcal{H}^{\theta}$ must be infinite. Thus we can apply Theorem 5.1 and the argument of Proposition 6.6 to conclude the statement.

The converse of Lemma 7.1 does not hold and, again, a counterexample is provided by Halphen vector fields (defined on $\mathbb{C}^{3}$ ) since they do have dicritical singularities at infinity and, still, possess hyperbolic leaves; see [26] or Section 7.2. For the time being, however, we finish this section by establishing Theorems $\mathrm{C}$ and C'.

Proof of Theorems C and C'. The proofs of both theorems begin with the following useful remark. Suppose that $X$ is a vector field defined on a compact Kähler manifold $N$ and let $p$ denote a singular point of $X$. Consider then the blow-up $\pi: \widehat{N} \rightarrow N$ of $N$ at $p$ along with the corresponding transform $\widehat{X}$ of $X$. Finally, denote by $\widehat{\mathcal{D}}$ (resp. $\mathcal{D})$ the foliation associated to $\widehat{X}$ (resp. $X$ ).

Since $X$ is complete, the regular leaves of $\mathcal{D}$ are quotients of $\mathbb{C}$. Owing to Brunella's theorem [6], the same applies to all the leaves of $\widehat{\mathcal{D}}$, including those contained in the exceptional divisor $\pi^{-1}(p)$. The foliation induced by $\widehat{\mathcal{D}}$ on $\pi^{-1}(p)$, however, depends only on the first nonzero homogeneous component $X_{2}$ of $X$ at $p$ which, in addition, has an isolated singularity at the origin. Thus, in local coordinates, we can apply Lemma 7.1 to $X_{2}$ to conclude that the leaves of its associated foliation are hyperbolic provided that it contains no dicritical singularity at infinity. This establishes the first item of Theorem C. As to Theorem C', note that the argument of Lemma 7.1, see also Theorem 5.1 and Proposition 6.6, applies to ensure that every leaf not passing through a dicritical singularity at infinity must 
be hyperbolic. Since all the leaves of the foliation associated to $X_{2}$ are known to be parabolic, Theorem $\mathrm{C}$ follows at once.

Finally, for the second item of Theorem C, just note that the same argument applies to the case of (hyperbolic) Halphen vector fields since these vector fields are known to have leaves that are isomorphic to the unit disc; see [26] or Section 7.2.

Remark 7.2 (A complement to Theorems C and C'). The purpose of this remark is to justify some of the additional claims made in the introduction and Section 2.1 concerning the statements of Theorems C and C' For this, we keep the preceding notation with $X=X_{k}+\cdots$ and denote by $\widehat{\mathcal{F}}_{\pi^{-1}(p)}^{k}$ the foliation induced on the exceptional divisor $\pi^{-1}(p)$ by $X_{k}$. When $X_{k}$ has a singular set of codimension 1 , we also set $X_{k}=P . Y^{\mathrm{cd} 2}$ as above.

The first claim made was that Theorem $\mathrm{C}$ and C' also hold when $X^{k}$ is a multiple of a semi-complete vector field $Y^{\mathrm{cd} 2}$ belonging to the Painlevé-Guillot lattice. To check this claim, we note that it ultimately concerns the leaves of $\widehat{\mathcal{F}}_{\pi^{-1}(p)}^{k}$. In this sense, we can replace $X_{k}$ by $Y^{\mathrm{cd} 2}$ provided that the latter vector field still is semi-complete.

The other previously made claim also concerns quadratic vector fields $X_{2}$ in the Painlevé-Guillot lattice. Denoting by $\mathcal{F}$ the foliation on $\mathbb{C}^{n}$ associated to $X_{2}$, assume that $\mathcal{F}$ has a dicritical singularity at infinity. Then, in Section 2.1, the following was stated:

Claim. The restriction of the vector field $X_{2}$ to a generic leaf $L$ of its associated foliation is either complete or conjugate to the vector field $x^{2} \partial / \partial x$ on all of $\mathbb{C}$. In the second case, the blow-up of $X$ at the origin has a dicritical singularity on the exceptional divisor and, moreover, the saturate of these dicritical singularities defines an open set where the vector field $X$ admits nonconstant first integrals.

To establish this claim, we proceed as follows. Consider the semi-complete vector field $X$ defined on $L$. Naturally we can assume $X$ is not complete otherwise the statement is immediate. Since $L$ is known to be a parabolic Riemann surface, if the restriction $X_{\mid L}$ of $X$ to $L$ is not complete, then the classification of semicomplete vector fields on $\mathbb{C}$ (see Section 2.2) ensures that $X_{\mid L}$ is conjugate to the vector field $x^{2} \partial / \partial x$ defined on $\mathbb{C}$. In turn, when a generic leaf of $\mathcal{F}$ has a singularity of type $x^{2} \partial / \partial x$, it must define a local separatrix for a singularity of $\widetilde{\mathcal{F}}$ on $\Delta_{0}$. The discussion in Section 6.2 about generic leaves of a foliation yielding separatrices for singular points applies here to ensure the existence of a dicritical singularity $p \in \Delta_{0}$ for $\tilde{\mathcal{F}}$. Finally, a singularity of type $x^{2} \partial / \partial x$ defines a neighborhood of $\infty$ for the domain of $\mathbb{C}$ where the time of $X_{\mid L}$ is defined. Since $X$ is semi-complete, it follows that $L$ can pass through $p$ only once. On the other hand, it is also known that $\widetilde{\mathcal{F}}$ is locally linearizable at $p$ and has integral eigenvalues. Therefore, around $p$, we can find a pair of meromorphic first integrals for $\widetilde{\mathcal{F}}$. These local first integrals can then be globalized by the foliation $\widetilde{\mathcal{F}}$ itself; the resulting global first integrals are well defined since the leaves of $\widetilde{\mathcal{F}}$ can intersect a small neighborhood of $p$ only once. The claim is proved. 


\subsection{Poincaré-type series and Halphen vector fields}

As a matter of fact, Halphen vector fields constitute a particularly remarkable example of semi-complete vector fields belonging to the Painlevé-Guillot lattice. Their associated geometry and dynamics is nicely described in [26]. These vector fields possess dicritical singularities in $\Delta_{\infty}$ (as well as in $\Delta_{0}$ ) together with leaves isomorphic (as Riemann surfaces) to the unit disc. As mentioned, this section is intended to discussing some aspects of these vector fields from the point of view emphasized in this paper. Inasmuch as the trajectories of $\mathcal{H}$ are finite for Halphen vector fields, the fact that they admit a suitable singular transverse foliation will still enable us to apply our renormalization techniques to these vector fields so as to derive additional information on them and, in particular, to show that the corresponding leaves are hyperbolic Riemann surfaces. The argument is therefore a bit different from the original proof of Guillot [26] which exploits the intimate connection between Halphen vector fields and the Lie algebra $\mathfrak{s l}(2, \mathbb{C})$ of PSL $(2, \mathbb{C})$. Although we can hardly improve on [26], we believe it is interesting to present here our point of view in these questions. The reader is referred to [26] for a full discussion.

In the sequel, we shall work on $\mathbb{C}^{3}$. Let $\mathcal{E}$ denote the radial vector field $\mathcal{E}=$ $x \partial / \partial x+y \partial / \partial y+z \partial / \partial z$ and set $Y=\partial / \partial x+\partial / \partial y+\partial / \partial z$. A quadratic homogeneous vector field $X$ is said to be a Halphen vector field if it satisfies $[Y, X]=2 \mathcal{E}$. It then follows that the triplet $Y, \mathcal{E}, X$ forms a Lie algebra isomorphic to $\mathfrak{s l}(2, \mathbb{C})$. With the terminology of Sections 3 and 4 , if $Y, X$, and $\mathcal{E}$ are identified with the vector fields they induce on $M$ and expressed in the corresponding coordinates $(x, y, z)$, then $\mathcal{E}$ becomes $z^{-1} \partial / \partial z$. In other words $X$ and $Y$ commute up to a vertical vector field. Thus $X$ preserves the projection on $\Delta_{\infty}$ of the foliation induced by $Y$. This means that the foliation $\mathcal{F}_{\infty}^{X}$ induced by $X$ on $\Delta_{\infty}$ is transverse to the foliation induced by $Y$ on $\Delta_{\infty}$. Since $Y$ is a constant vector field, the foliation it induces on $\Delta_{\infty}$ is simply a linear pencil of rational curves. Summarizing $\mathcal{F}_{\infty}^{X}$ is transverse to a linear pencil of rational curves.

Once the above observation is made, it is easy to work out the structure of $\mathcal{F}_{\infty}^{X}$. It leaves exactly 3 projective lines $C_{1}, C_{2}$ and $C_{3}$ invariant and these 3 lines intersect mutually at a radial singularity $P \in \Delta_{\infty}$. Indeed, the eigenvalues of $\mathcal{F}_{\infty}^{X}$ at $P$ are 1 and 1 while the eigenvalues of $\mathcal{F}^{X}$ (the foliation induced by $X$ on all of $M$ ) at $P$ are 1,1 , and -1 (the -1 eigenvalue is associated with the direction transverse to $\left.\Delta_{\infty}\right)$. Also, if $P^{\prime} \in \Delta_{0}$ is the singularity of $\mathcal{F}^{X}$ whose fiber $\mathcal{P}_{0}^{-1}\left(P^{\prime}\right)$ coincides with the fiber $\mathcal{P}_{\infty}^{-1}(P)$, then the eigenvalues of $\mathcal{F}^{X}$ at $P^{\prime}$ are 1,1 , and 1 . For $i=1,2,3$, let $p_{i}$ and $q_{i}$ denote the remaining two singularities of $\mathcal{F}^{X}$ over $C_{i}$. We assume that $X$ is semi-complete though this is not indispensable in what follows. This ensures that the eigenvalues of $\mathcal{F}^{X}$ at $p_{i}$ (resp. $q_{i}$ ) have the form $-1,-1$, and $m_{i}$ (resp. $-1,-1$, and $-m_{i}$ ), with $m_{i} \in \mathbb{N}^{*}$. The converse also holds and is Halphen's main result, which is revisited by Guillot in [26]. This last point will be taken for granted in our discussion. Note that it is also easy to check that $\mathcal{F}^{X}$ is locally linearizable about $p_{i}$ (resp. $q_{i}$ ). Finally, note that the convention used above concerning the order of the eigenvalues of $\mathcal{F}^{X}$ at $p_{i}$ (resp. $q_{i}$ ) is such that the first eigenvalue corresponds to the vertical direction $z$, the second to the curve $C_{i}$ and 
the third to a direction contained in $\Delta_{\infty}$ and transverse to $C_{i}$. This convention is slightly different from that in [26] since the eigenvalue associated with the direction $z$ is -1 rather than 1 . This change of sign is due to the fact that we consider singularities in $\Delta_{\infty}$ whereas Guillot considers singularities in $\Delta_{0}$.

The dynamics of $\mathcal{F}_{\infty}^{X}$ is fully encoded in its global holonomy group with respect to a fixed line $\bar{C}$ in the above mentioned linear pencil that is transverse to $\mathcal{F}_{\infty}^{X}$. The preceding also shows that this holonomy group coincides with the subgroup of PSL $(2, \mathbb{C})$ generated by three elements $\xi_{1}, \xi_{2}$, and $\xi_{3}$ which are associated with the local holonomy maps arising from each of the three invariant lines. In particular, we have

$$
\xi_{1} \xi_{2} \xi_{3}=\xi_{1}^{m_{1}}=\xi_{2}^{m_{2}}=\xi_{3}^{m_{3}}=\mathrm{id} .
$$

In other words, this is a triangle group whose dynamics on $S^{2}$ is well known. Provided that

$$
\frac{1}{m_{1}}+\frac{1}{m_{2}}+\frac{1}{m_{3}}<1,
$$

this group is conjugate to a subgroup of PSL $(2, \mathbb{C})$ and thus it leaves a circle $\Lambda_{\infty} \subset$ $S^{2} \simeq \bar{C}$ invariant. Furthermore each connected component of $\bar{C} \backslash \Lambda_{\infty}$ is invariant under the action. In fact, on these components the action is properly discontinuous whereas it is minimal when restricted to the circle $\Lambda_{\infty}$ itself. Similarly, in the case where $1 / m_{1}+1 / m_{2}+1 / m_{3}=1$, the resulting groups are well-known groups of affine diffeomorphisms associated to special tilings of the plane. When $1 / m_{1}+$ $1 / m_{2}+1 / m_{3}>1$ the resulting group is, indeed, finite and thus it is easy to see that all leaves are compact.

Remark 7.3. A motive for caution with respect to the preceding concerns the fact that the quasi-isometric type of the holonomy group does not priori determine the quasi-isometric type of the leaves of $\mathcal{F}^{X}$ since the latter are not everywhere transverse to the associated fibration. In addition to the existence of singularities, there are 3 fibers of this fibration that are invariant under $\mathcal{F}^{X}$. Therefore it is not clear that the leaves of $\mathcal{F}^{X}$ must be hyperbolic once the estimate (7.2) has been verified.

A similar picture is valid for the foliation $\mathcal{F}^{X}$ of $M$ associated with $X$. Clearly $\mathcal{F}^{X}$ is transverse to the codimension 1 foliation defined by the cone over the leaves of $\mathcal{F}_{\infty}^{Y}$ and its dynamics is again encoded in the corresponding holonomy group. This group is still generated by the local holonomy with respect to $M$ of each of the three mentioned invariant lines. However, each of the three generators is now realized as an automorphism $\Xi_{i}$ of $\mathbb{F}_{1}$, the line bundle over $\mathbb{C P}^{1}$ with Chern number 1 . In our context, this line bundle is the cone over $\bar{C} \subset \Delta_{\infty}$. Moreover, $\mathbb{F}_{1}$ can be obtained by gluing together two copies of $\mathbb{C} \times \mathbb{C}$ with coordinates $(w, z)$ and $\left(w^{\prime}, z^{\prime}\right)$ according to the equation $\left(w^{\prime}, z^{\prime}\right)=(1 / w, w z)$. The second coordinate of the first copy can be identified with the previous affine coordinate $z$ for $\mathbb{C}^{3}$. The automorphism $\Xi_{i}$ fixes the zero section and thus it can be expressed in these coordinates as $\Xi_{i}(w, z)=\left(\xi_{i}(w), B_{i}(w) z\right)$ where $\xi_{i}(w)$ is a projective transformation. 
Furthermore, it is also known that $\Xi_{i}^{m_{i}}=$ id on $\mathbb{F}_{1}$ and $\xi_{i}^{m_{i}}=\mathrm{id}$ on $\bar{C}$. Thus

$$
B_{i}(w) \times B_{i}\left(\xi_{i}(w)\right) \times \cdots \times B_{i}\left(\xi_{i}^{m_{i}-1}(w)\right)=1 .
$$

Next, recall that the action of an element of the Möbius group has a natural extension to $\mathbb{F}_{1}$ given by the multiplication in the fibers by the square root of its derivative. In other words, if $\xi$ is a projective transformation, then its extended action on a pair $(w, z) \in \mathbb{F}_{1}$ is

$$
\xi .(w, z)=\left(\xi(w), \sqrt{\xi^{\prime}(w)} z\right) .
$$

Note that the square root of the derivative of a projective transformation is well defined so that the claim follows from observing that $\mathbb{F}_{1} \otimes \mathbb{F}_{1}$ is isomorphic to the tangent bundle of $\bar{C}$. In particular, a more explicit expression for $\Xi_{i}$ can be derived as follows. Let $q_{i}, q_{i+1}(\neq 0, \infty)$ denote the two fixed points of $\xi_{i}$ in $\bar{C}$. The transformation

$$
\sigma_{i}(w)=\frac{w-q_{i}}{w-q_{i+1}} ; \quad \sigma_{i}^{-1}(w)=\frac{q_{i+1} w-q_{i}}{w-1}
$$

conjugates $\xi_{i}$ to a projective transformation fixing 0 and $\infty$. In the coordinate $\bar{w}$, $\xi_{i}$ must take on the form $\bar{w} \mapsto k_{i}^{2} \bar{w}$ where $k_{i}^{2}=e^{2 \pi \sqrt{-1} / m_{i}}$ since $\xi_{i}^{m_{i}}=$ id on $\bar{C}$. Furthermore, in the coordinate $\bar{w}$, it is clear that $B_{i}(\bar{w})$ must be constant so as to allow $\Xi_{i}$ to have a holomorphic extension to a fibered neighborhood of $\infty$. Setting $B_{i}(\bar{w})=B_{i}$ for this particular choice of coordinates, we conclude that the expression of $\Xi_{i}$ in the initial coordinate $w$ is given by

$$
\begin{aligned}
\Xi_{i}(w, z) & =\left(\xi_{i}(w), B_{i}(w) z\right) \\
= & \left(\sigma_{i}^{-1}\left(k_{i}^{2} \sigma_{i}(w)\right), \sqrt{\left.\left(\sigma_{i}^{-1}\right)^{\prime}\right|_{k_{i}^{2} \sigma_{i}(w)}} B_{i} \sqrt{\sigma_{i}^{\prime}(w) z}\right) \\
= & \left(\frac{\left(k_{i}^{-1} q_{i}-k_{i} q_{i+1}\right) w+q_{i} q_{i+1}\left(k_{i}-k_{i}^{-1}\right)}{\left(k_{i}^{-1}-k_{i}\right) w+\left(k_{i} q_{i}-k_{i}^{-1} q_{i+1}\right)}, \frac{k_{i}^{-1} B_{i}\left(q_{i}-q_{i+1}\right) z}{\left(k_{i}^{-1}-k_{i}\right) w+\left(k_{i} q_{i}-k_{i}^{-1} q_{i+1}\right)}\right) \\
= & \left(\xi_{i}(w), k_{i}^{-1} B_{i} \sqrt{\xi_{i}^{\prime}(w)} z\right) .
\end{aligned}
$$

The above formulas are going to enable us to understand the solutions of Halphen vector fields from the point of view developed in this article. We first consider the special case where $1 / m_{1}+1 / m_{2}+1 / m_{3}=1$. In this case the three projective transformations $\xi_{1}, \xi_{2}$, and $\xi_{3}$ share a common fixed point. Choosing coordinates $(w, z)$ where this point is $\infty$ it follows that $\xi^{\prime}(w)$ is constant and thus $\Xi_{i}(w, z)=\left(\xi_{i}(w), A_{i} z\right)$ for certain constants $A_{i}, i=1,2,3$. As a sort of converse to Theorem 5.1, we obtain the following:

Proposition 7.4. Suppose that $1 / m_{1}+1 / m_{2}+1 / m_{3}=1$. Then all the leaves of $\mathcal{F}^{X}$ are uniformized by $\mathbb{C}$ as Riemann surfaces.

Proof. The proof is simple. Let $L$ be a generic leaf of $\mathcal{F}_{X}$ and let $\mathbb{F}_{1}$ be as above. Because all the $A_{i}$ 's are constant, the intersection points $p_{1}, p_{2} \ldots$ of $L$ with $\mathbb{F}_{1}$ 
have their distance to $\Delta_{\infty}$ bounded from below by a positive constant. Thus the time-form $d T_{L}$ induced by $X$ on $L$ is uniformly bounded on a neighborhood $W$ of $\left\{p_{1}, p_{2} \ldots\right\}$. Consider now the maximal domain $\mathbf{U} \subseteq \mathbb{C}$ of a solution $\phi$ of $X$. Suppose for the sake of a contradiction that $\mathbb{C} \backslash \mathbf{U} \neq \emptyset$ and choose a point $T \in \mathbb{C}$ lying on the boundary $\partial \mathbf{U}$ of $\mathbf{U}$. Finally let $t_{1}, t_{2}, \ldots$ be a sequence of points in $\mathbf{U}$ converging to $T$. Since $\mathcal{F}_{X}$ is transverse to a fibration, we can assume without loss of generality that $\phi\left(t_{j}\right)$ lies in $W$. Since $d T_{L}$ is uniformly bounded on $W$, it follows that, for every $j$ fixed, $\phi$ is defined on a disc centered at $t_{j}$ and having a uniform (positive) radius. This is however impossible since $t_{j} \rightarrow T \in \partial \mathbf{U}$. The proposition is proved.

Henceforth we focus on the more interesting case where $\xi_{1}, \xi_{2}$, and $\xi_{3}$ generate a hyperbolic triangle group, i.e., on the case where $1 / m_{1}+1 / m_{2}+1 / m_{3}<1$. The fixed points of $\xi_{1}, \xi_{2}$, and $\xi_{3}$ are three (pairwise distinct) points $q_{1}, q_{2}, q_{3} \in \bar{C}$. This means that for each $i \in\{1,2,3\}$, the projective transformation $\xi_{i}$ fixes the points $q_{i}$ and $q_{i+1}$ (where $q_{3+1}=q_{1}$ ). In the present case, there is no coordinate $w$ where all the $\xi_{i}$ become affine maps. Thus we shall need to work with the full information provided by the action of the group generated by $\Xi_{1}, \Xi_{2}$, and $\Xi_{3}$ on $\mathbb{F}_{1}$. We will show that the geometry of the leaves is related to the Poincaré series with exponent $1 / 2$. Next, denote by $\Gamma$ the group generated by $\xi_{1}, \xi_{2}, \xi_{3}$ and consider its Cayley graph with respect to the generating set given by $\xi_{1}, \xi_{2}, \xi_{3}$, and their inverses. Choose a geodesic ray $\gamma_{0}=\mathrm{id}, \gamma_{1}, \gamma_{2}, \ldots$ in the Cayley graph going from the identity to an end of the graph. We have:

Proposition 7.5. Suppose the the holonomy group is a hyperbolic triangle group. Let $L$ denote a leaf of $\mathcal{F}_{X}$ passing through a point $\left(w_{0}, z_{0}\right)$. As a Riemann surface $L$ is hyperbolic provided that the series

$$
S\left(w_{0}\right)=\sum_{j=0}^{\infty}\left\|\gamma_{j}\left(w_{0}\right)\right\|^{1 / 2}
$$

converges for more than one geodesic ray. If this series diverges for all geodesic rays as above then $L$ is a quotient of $\mathbb{C}$.

Proof. The proof consists in elaborating on the argument used in Proposition 7.4. Again denote by $\mathbf{U}$ the maximal domain of definition of the solution $\phi$ of $X$ satisfying $\phi(0)=\left(w_{0}, z_{0}\right), z_{0} \neq 0$. Denote by $L_{\infty}$ the projection of $L$ on $\Delta_{\infty}$. By virtue of the structure of the foliation $\mathcal{F}_{\infty}^{X}$ on $\Delta_{\infty}$, we know that $L_{\infty}$ is a ramified covering of $\mathbb{C P}^{1}$ where the ramification points lie over three points of $\mathbb{C P}^{1}$ (identified with the three invariant fibers of $\left.\mathcal{F}_{\infty}^{X}\right)$. We can then think of $L_{\infty}$ as being the universal covering of $\mathbb{C} P(1)$ minus 3 points modulo adding to it the ramification points. In particular, there is natural sense in considering fundamental domains in $L_{\infty}$. The leaf $L_{\infty}$ can then be considered as the union of the corresponding fundamental domains $L_{\infty}^{(0)}, L_{\infty}^{(1)}, \ldots$ such that $L_{\infty}^{(j)}=\gamma_{j}\left(L_{\infty}^{(0)}\right)$. These domains have natural lifts to the leaf $L \subset M \backslash\left(\Delta_{0} \cup \Delta_{\infty}\right)$ which, modulo relabeling, will be denoted by $L^{(0)}, L^{(1)}, \ldots$ so that $L^{(j)}=\Gamma_{\underline{j}}\left(L^{(0)}\right)$ where $\Gamma_{j}$ is the automorphism of $\mathbb{F}_{1}$ corresponding to the action of $\gamma_{j}$ on $\bar{C}$. 
On $L$ (or on its universal covering if necessary), we define the map

$$
\mathcal{D}_{L}(p)=\int_{\left(w_{0}, z_{0}\right)}^{p} d T_{L}
$$

where $d T_{L}$ stands for the time-form induced on $L$. Since $X$ is semi-complete, $\mathcal{D}_{L}$ provides a diffeomorphism from the (universal covering of) $L$ to $\mathbf{U}$. Let $U_{(j)}$ be the image of $L^{(j)}$ under $\mathcal{D}_{L}$. The above assertion implies that the sets $U_{(j)}$ tile $\mathbf{U}$ without overlapping and modulo adding the images of ramification points (involved in the preceding definition of the fundamental domains $L_{\infty}^{(0)}, L_{\infty}^{(1)}, \ldots$ ). Next, recall that the affine structure on $L_{\infty}$ is uniformly bounded (from below and from above). Combining this fact with the expression for $d T_{L}$ arising from 3.1, with $d=2$, there follows the existence of sequences $\left\{r_{j}\right\}$ and $\left\{R_{j}\right\}, 0<r_{j}<R_{j}$, $j=1,2, \ldots$, satisfying the conditions below.

1. There are constants $0<c<C$, independent of $j$, such that

$$
c\left\|\pi_{2}\left(\Xi_{j}\left(w_{0}, z_{0}\right)\right)\right\| r_{j}<R_{j}<C\left\|\pi_{2}\left(\Xi_{j}\left(w_{0}, z_{0}\right)\right)\right\| r_{j},
$$

where $\pi_{2}$ stands for the projection on the second coordinate (i.e., the fiber of $\left.\mathbb{F}_{1}\right)$.

2. The image of $U_{(j)}$ under $\mathcal{D}_{L}$ contains a ball of radius $r_{j}$ centered at the point $\mathcal{D}_{L}\left(\Xi_{j}\left(w_{0}, z_{0}\right)\right)$. Similarly this image is contained in a ball of radius $R_{j}$ centered at $\mathcal{D}_{L}\left(\Xi_{j}\left(w_{0}, z_{0}\right)\right)$.

It then becomes clear that $\mathbf{U}$ must be all of $\mathbb{C}$ provided that the series

$$
\sum_{j=0}^{\infty} \| \pi_{2}\left(\Xi_{j}\left(w_{0}, z_{0}\right) \|\right.
$$

diverges for every geodesic ray. Conversely, if this series converges, then we can easily construct a small piece of a continuum contained in the boundary of $\mathbf{U} \subset \mathbb{C}$. Thus $\mathbf{U}$ must be a hyperbolic domain so that $L$ itself must be a Riemann surface covered by the unit disc. To conclude the proof of the proposition it is therefore sufficient to check that the series $\sum_{j=0}^{\infty}\left\|\pi_{2}\left(\Xi_{j}\left(w_{0}, z_{0}\right)\right)\right\|$ converges (resp. diverges) if and only if so does the reduced Poincaré series in the statement. This is however clear since $\left|k_{i}^{-1} B_{i}\right|=1$ as an immediate consequence of (7.3). The proof of the proposition is finished.

Next we show that the series (7.4) always converges provided that $w_{0} \in \bar{C}$ $\Lambda_{\infty}$. Some indications concerning the behavior of this series in the case $w_{0} \in \Lambda_{\infty}$ will also be provided. As mentioned the series in question differs from the usual Poincaré series since the summation does not run over the entire group but only over those elements belonging to a chosen geodesic ray. Indeed the full Poincaré series of $\Gamma$ with exponent $1 / 2$ diverges as follows from well-known results due mainly to Sullivan (see [36] for an overview of the standard theory).

Lemma 7.6. The series (7.4) converges provided that $w_{0} \in \bar{C} \backslash \Lambda_{\infty}$. 
Proof. The argument is simple. Consider the action of $\Gamma$ in the $w$-plane. This action preserves a circle identified with $\Lambda_{\infty}$ so that $\Gamma$ is identified with a Fuchsian group, i.e., a discrete group of automorphisms of the hyperbolic disc. Next, since $\Gamma$ acts on the hyperbolic disc, it is easy to see that the convergence of this series does not depend on $w_{0}$. In other words, the series (7.4) converges for $w_{0}$ if and only if it converges at 0 . It is then sufficient to check that the series converges for $w_{0}=0$ (identified with the origin). For this consider again the geodesic ray $\gamma_{0}=\mathrm{id}, \gamma_{1}, \gamma_{2}, \ldots$ in the Cayley graph of $\Gamma$ and set $a_{j}=\gamma_{j}(0)$. In the Cayley graph the distance between the identity and $\gamma_{j}$ is obviously $j$. The existence of a quasiisometry between this graph and the hyperbolic disc implies that the hyperbolic distance $d_{H}\left(0, \gamma_{j}(0)\right)$ between 0 and $\gamma_{j}(0)$ satisfies $c . j<d_{H}\left(0, \gamma_{j}(0)\right)<C . j$ for appropriate uniform constants $C>c>0$. The standard formula for the length of a minimizing geodesic in the hyperbolic unit disc joining 0 to a point $a$ of this disc (naturally satisfying $\|a\|<1$ ) yields

$$
\frac{e^{c . j}-1}{e^{c . j}+1} \leq\left\|a_{j}\right\| \leq \frac{e^{C . j}-1}{e^{C . j}+1} .
$$

On the other hand the coefficients of the hyperbolic metric at 0 and at $a_{j}$ allow us to obtain a formula for the derivative of $\gamma_{j}$ at 0 . Combined with the above estimates this formula gives

$$
\left\|\gamma_{j}^{\prime}(0)\right\| \leq \frac{4 e^{C \cdot j}}{\left(1+e^{C \cdot j}\right)^{2}}
$$

and thus $\left\|\gamma_{j}^{\prime}(0)\right\|^{1 / 2} \leq 2 e^{C \cdot j / 2} /\left(e^{C \cdot j}+1\right)$. The convergence of the series (7.4) follows immediately.

To close this discussion we briefly indicate the behavior of the series (7.4) for points $w_{0}$ lying in $\Lambda_{\infty}$. Since $\gamma_{j}$ takes 0 to $a_{j}$, it follows that $\gamma_{j}(w)=e^{2 \pi i \theta}(w+$ $\left.a_{j}\right) /\left(1+\bar{a}_{j} w\right)$ for some $\theta \in[0,1)$. In particular

$$
\left\|\gamma_{j}^{\prime}(w)\right\|=\frac{1-\left\|a_{j}\right\|^{2}}{\left(1+\bar{a}_{j} w\right)^{2}}
$$

Because $\Gamma$ is discrete, it follows that $\left\|a_{j}\right\| \rightarrow 1$ as $j \rightarrow+\infty$. Set $a_{j}=\left\|a_{j}\right\| e^{i \theta_{j}}$ and $w_{0}=e^{-i \theta_{j}-\pi+\alpha_{j}}$ so that

$$
\left\|\gamma_{j}^{\prime}\left(w_{0}\right)\right\|=\frac{1-\left\|a_{j}\right\|^{2}}{1+\left\|a_{j}\right\|^{2}-2\left\|a_{j}\right\| \cos \left(\alpha_{j}\right)} .
$$

Next note that $\left\|\gamma_{j}^{\prime}\left(w_{0}\right)\right\|>1 / 2$ as long as $\cos \left(\alpha_{j}\right)>\left\|a_{j}\right\|$. In particular, if there are infinitely many indices $j$ satisfying this condition the corresponding series diverges. In this case there is a subsequence of $\left\{a_{j}\right\}$ converging almost radially to $-w_{0}$. Conversely if the denominator in (7.5) is bounded from below by some positive constant, then the argument used in Lemma 7.6 ensures again the convergence of the series (7.4). In general we are led to a finer analysis taking into account the conic approximation of $-z_{0}$ by the sequence $a_{j}=\gamma_{j}(0)$. Not surprisingly the behavior of series (7.4) on $\Lambda_{\infty}$ depends on the initial point $w_{0}$; for some values of $w_{0}$ it diverges whereas for others it converges. 
Most properties of Halphen vector fields are encoded in the extended dynamics of the group generated by $\Xi_{1}, \Xi_{2}$, and $\Xi_{3}$ on $\mathbb{F}_{1}$. For example the study of first integrals for Halphen vector fields amounts to searching for functions that are invariant under this action. Hence, on each connected component of $\bar{C} \backslash \Lambda_{\infty}$ it is not hard to construct automorphic functions for this group so that on the corresponding open sets of $M$ the Halphen vector field admits a holomorphic first integral. It is also not very hard to check that the Halphen vector field does not admit a holomorphic (or meromorphic) first integral on the set corresponding to $\Lambda_{\infty}$ (which has real dimension 5 ). However, on the latter set, there is a realvalued first integral for the equation that is actually globally defined on $M$. We shall not pursue this discussion here not only because Halphen vector fields were studied in detail in [26] but also because the relevant issues do not fit in the general context of the main ideas of this paper.

\section{Appendix: some problems}

In closing, we suggest some problems for which the method developed in this work might provide some insight.

Equations with the Painlevé property. It is clear that our methods are well designed for investigating these equations, specially if meromorphic solutions defined on all of $\mathbb{C}$ are targeted. Since Nevanlinna theory has become an important tool for studying these questions, it would be interesting to compare both approaches which, as suggested by Theorem A', may have some nontrivial points of contact. Here, we remind the reader that our methods apply equally well to rational, besides polynomial, vector fields.

Also many special equations including Painlevé equations admit a formulation involving associated transverse fibrations. The domains of definition of their solutions can then be studied by adapting the argument used in Section 7.2 for handling the case of Halphen equations. For example, consider the Painlevé-VI (P-VI) case which contains the remaining Painlevé equations as particular cases. The standard Hamiltonian formulation of P-VI allows one to consider its holonomy group at infinity, as in [30], where this holonomy group is shown to be virtually Abelian. By construction, this group consists of holomorphic diffeomorphisms of $\mathbb{C}^{2}$. The study of the corresponding dynamics should then recover, in particular, the domain of definition of the equations in question as well as yielding new insight into the way they bifurcate as the parameters vary. This certainly relates to several other aspects of the Painlevé equations such as global linearization and Riemann-Hilbert correspondence.

Confinement questions for real equations. These problems were brought to our attention by F. Cano and C. Roche. Let us consider the case of singular points of real analytic vector fields as in [13] and [14]. Several important issues appearing in the study of these singular points, such as the existence of iterated tangents, 
depend strongly on having suitable estimates on the speed with which a solution converges to the origin. Though these problems are real rather than complex, our method can provide information in certain cases. We explain the main changes needed for this.

The central issue is that the exceptional divisor in this context is obtained by a real blow-up and the foliation induced on it has real dimension 1 as opposed to complex dimension 1. Thus there is no freedom to choose any steepest descent direction, indeed, we can only follow the given trajectory in a chosen orientation. However, under some assumptions concerning the first nonzero homogeneous component of the vector field, it is possible to guarantee a normal contractive holonomy such as that considered in Section 3.

Once this contraction is established, the fact that we are dealing with real solutions, rather than with complex ones, becomes an advantage for two main reasons which are more easily understood in dimension 3 . The first one has to do with Lemma 3.5. Recall that $\Delta_{\infty}^{(x, y)}$ consists of source singularities for $\mathcal{H}$ whereas $\Delta_{0}^{(x, y)}$ is constituted by sink singular points of $\mathcal{H}$. This is the main reason why we have worked with $\Delta_{\infty}^{(x, y)}$ rather than with $\Delta_{0}^{(x, y)}$; the fact that $\mathcal{H}$ has source singularities at $\Delta_{\infty}^{(x, y)}$ is fundamental for the proof of Lemma 3.10 and hence for Theorem 4.7. Nonetheless, in the real case, it often occurs that these trajectories remain in a compact part of the complement of the line at infinity inside $\Delta_{0}$. Therefore, modulo appropriate assumptions satisfied by many foliations, the statement of Theorem 4.7 remains valid in the present setting. When working in dimension 3 , the situation becomes much better; dealing with real equations, the divisor playing the role of $\Delta_{0}$ is no longer the complex projective plane but the real projective plane. The asymptotic behavior of the real foliation induced on the analogue of $\Delta_{0}$ is therefore easily described by the standard Poincaré-Bendixson theory. It is then reasonable to expect it to provide detailed information on the structure of the singular point in question. It would be nice to know if the theory proposed by F. Cano, R. Moussu, and their collaborators can be furthered by this type of analysis.

We mention that the study of complete real vector fields on $\mathbb{R}^{3}$ may be approached from this point of view as was pointed out to us by A. Guillot. In particular, this type of idea applies to Lorenz equation where the presence of a very special saddle node singularity in $\Delta_{\infty}$ seems to organize much of the information concerning the corresponding dynamics. Similar ideas concerning Lorenz systems were also considered by X. Gómez-Mont and, independently, by J.-P. Ramis.

Actions of SL $(2, \mathbb{C})$. In [26], A. Guillot classified compact complex manifolds of dimension 3 that are quasihomogeneous under an action of SL $(2, \mathbb{C})$. This means that the action of SL $(2, \mathbb{C})$ fails to be locally free in the complement of a Zariski open set. The starting point of Guillot's work is precisely to study homogeneous vector fields $X$ satisfying the equation $[Y, X]=2 \mathcal{E}$ where $\mathcal{E}=x \partial / \partial x+y \partial / \partial y+$ $z \partial / \partial z$ and $Y=\partial / \partial x+\partial / \partial y+\partial / \partial z$. These vector fields $X$ are by definition Halphen vector fields. The same equation can be considered in every dimension to yield higher dimensional analogues of Halphen vector fields that are naturally related to $\mathrm{SL}(2, \mathbb{C})$-actions. 
Let $\mathcal{E}$ and $Y$ continue to denote the constant and the radial vector fields in dimension $n$. The previous discussion shows that $X$ still induces a foliation on $\Delta_{\infty}$ transverse to the linear pencil obtained from $Y$. The interesting novelty appearing in dimensions $\geq 4$ is that the basis of this pencil is isomorphic to $\mathbb{C P}^{n-2}$ which inherits a nontrivial foliation induced by $X$ since $n \geq 4$. We do not know how wild this foliation can be, but an understanding of its dynamics would allow us to generalize the arguments given in Section 7.2 and extend the results of Halphen and Guillot.

Singular points of vector fields on complex Kähler 3-folds. Let $X$ be a holomorphic vector field defined on a compact Kähler manifold $M$ of dimension 3 and consider a singular point $p \in M$ of $X$. Denote by $X_{d}$ the first nonzero homogeneous component of $X$ at $p$. It is known that $X_{d}$ is a (homogeneous) semi-complete vector field on all of $\mathbb{C}^{3}$. The problem is then to classify all possible models for $X_{d}$. It turns out that this problem is equivalent to finding all possible normal forms for the top degree homogeneous component of a complete polynomial vector field on $\mathbb{C}^{3}$.

Consider the foliation $\widetilde{\mathcal{F}}_{\infty}$ induced on $\Delta_{\infty}$ by $X_{d}$ and assume once and for all that $\widetilde{\mathcal{F}}_{\infty}$ possesses some Zariski dense leaf. Since all leaves of $\widetilde{\mathcal{F}}_{\infty}$ are parabolic Riemann surfaces, we can apply the McQuillan theorem, as formulated in [8], to conclude that $\widetilde{\mathcal{F}}_{\infty}$ is transverse to a pencil of genus 0 or 1 . The idea is then to resort again to arguments similar to those developed in Section 7.2 to work out the structure of $\widetilde{\mathcal{F}}_{\infty}$. The first thing to be proved is that the global holonomy arising from $\widetilde{\mathcal{F}}_{\infty}$ is conjugate to a subgroup of the affine group of $\mathbb{C}$. In particular it has a fixed point corresponding to an algebraic curve $C$ invariant under $\widetilde{\mathcal{F}}_{\infty}$. Next we should consider the extended holonomy of $\widetilde{\mathcal{F}}$ taking values on the group of automorphisms of $F_{1}$ as was done previously. The resulting group will still be elementary and it must be compatible with the affine structure induced on $C$ which is necessary uniformizable. Finally the cases in which the genus of $C$ is 0 or 1 must be considered separately. The more interesting case corresponds to genus 0 since there are more possibilities for the affine structure on $C$. Besides rational orbits, we must expect to find the elliptic orbifolds associated with the triangle groups $(2,2, \infty),(2,3,6),(2,4,4)$ and $(3,3,3)$ in addition to the orbifold $(2,2,2,2)$ which appear in connection with the classical integrable equations of the Euler spinning top.

\section{References}

[1] Ablowitz, M.J., Ramani, A. And Segur, H.: A connection between nonlinear evolution equations and ordinary differential equations of P-type, I. J. Math. Phys. 21 (1980), 715-721.

[2] Ablowitz, M. J., Ramani, A. And Segur, H.: A connection between nonlinear evolution equations and ordinary differential equations of P-type, II. J. Math. Phys. 21 (1980), 1006-1015. 
[3] Andersen, E. And Lempert, L.: On the group of holomorphic automorphisms of $\mathbb{C}^{n}$. Inv. Math. 110 (1992), no. 2, 371-388.

[4] Arnol'd, V. I. And IL'Yashenko, Yu. S.: Ordinary differential equations. In Encyclopedia of Math. Sciences 1, Dynamical Systems I, 1-148. Springer, Berlin, 1988.

[5] Bonatti, C., Gómez-Mont, X. And Vila-Freyer, R.: Statistical behaviour of the leaves of Riccati foliations. Ergodic Theory Dynam. Systems 30 (2010), no. 1, 67-96.

[6] Brunella, M.: Plurisubharmonic variation of the leafwise Poincaré metric. Internat. J. Math. 14 (2003), no. 2, 139-151.

[7] Brunella, M.: Complete polynomial vector fields on the complex plane. Topology 43 (2004), no. 2, 433-445.

[8] Brunella, M.: Birational geometry of foliations. Publicações Matemáticas do IMPA, Instituto de Matemática Pura e Aplicada (IMPA), Rio de Janeiro, 2004.

[9] Bustinduy, A.: On the entire solutions of a polynomial vector fields on $\mathbb{C}^{2}$. Indiana Univ. Math. J. 53 (2004), 647-666.

[10] Bustinduy, A. And Giraldo, L.: Completeness is determined by any non-algebraic trajectory. Adv. Math. 231 (2012), no. 2, 664-679.

[11] Buzzard, G. And Fornaess, J.-E.: Complete holomorphic vector fields and time-1 maps. Indiana Univ. Math. J. 44 (1995), no. 4, 1175-1182.

[12] Camacho, C., Cano, F. And Sad, P.: Absolutely isolated singularities of holomorphic vector fields. Invent. Math. 98 (1989), 351-369.

[13] Cano, F., Moussu, R. and Rolin, J.P.: Non-oscillating integral curves and valuations. J. Reine Angew. Math. 582 (2005), 107-141.

[14] Cano, F., Moussu, R. And Sanz, F.: Oscillation, spiralement, tourbillonnement. Comment. Math. Helv. 75 (2000), no. 2, 284-318.

[15] Cerveau, D. And ScÁrdua, B.: Complete polynomial vector fields in two complex variables. Forum Math. 17 (2005), 407-430.

[16] Forness, J.-E. ANd Grellier, S.: Exploding orbits of holomorphic Hamiltonians and of complex contact structures. C.R. Acad. Sci. Paris, Sér. I Math. 319 (1994), no. $6,553-557$.

[17] Fornæss, J.-E. And Sibony, N.: Riemann surface laminations with singularities. J. Geom. Anal. 18 (2008), no. 2, 400-442.

[18] Fornæss, J.-E. And Sibony, N.: Unique ergodicity of harmonic currents on singular foliations of $\mathbb{P}^{2}$. Geom. Funct. Anal. 19 (2010), no. 5, 1334-1377.

[19] Forstneric, F.: Actions of $(\mathbb{R},+)$ and $(\mathbb{C},+)$ on complex manifolds. Math. Z. 223 (1996), 123-153.

[20] Ghys, É.: L'invariant de Godbillon-Vey. Séminaire Bourbaki, Vol. 1988/89. Astérisque 177-178 (1989), Exp. No. 706, 155-181.

[21] Ghys, É. and Rebelo, J.-C.: Singularités des flots holomorphes. II. Ann. Inst. Fourier (Grenoble) 47 (1997), no. 4, 1117-1174.

[22] Gómez-Mont, X.: Personal communication.

[23] Guillot, A.: Champs quadratiques uniformisables. Thesis, ENS-Lyon, 2001.

[24] Guillot, A.: Sur les exemples de Lins Neto de feuilletages algébriques. C. R. Math. Acad. Sci. Paris 334 (2002), no. 9, 747-750. 
[25] Guillot, A.: Semicompleteness of homogeneous quadratic vector fields. Ann. Inst. Fourier (Grenoble) 56 (2006), no. 5, 1583-1615.

[26] Guillot, A.: Sur les équations d'Halphen et les actions de $\mathrm{SL}_{2}(\mathbb{C})$. Publ. Math. Inst. Hautes Etudes Sci. 105 (2007), no. 1, 221-294.

[27] Guillot, A.: Some generalizations of Halphen's equations. Osaka J. Math. 48 (2011), no. 4, 1085-1094.

[28] Guillot, A.: The geometry of Chazy's homogeneous third-order differential equations. Funkcial. Ekvac. 55 (2012), no. 1, 67-87.

[29] Guillot, A. and Rebelo, J. C.: Semicomplete meromorphic vector fields on complex surfaces. J. Reine Angew. Math. 667 (2012), 27-65.

[30] Ben Hamed, B., Gavrilov, L. and Klughertz, M.: The holonomy group at infinity of the Painlevé VI equation. J. Math. Phys. 53 (2012), no. 2, 022701, 15 pp.

[31] Ince, E. L.: Ordinary differential equations. Dover Publications, New York, 1944.

[32] Langevin, R. And Rosenberg, H.: Quand deux sous-variétés sont forcées par leur géom'etrie à se rencontrer. Bull. Soc. Math. France 120 (1992), no. 2, 227-235.

[33] Phillips, A. and Sullivan, D.: Geometry of leaves. Topology 20 (1981), no. 2, 209-218.

[34] Rebelo, J. C.: Singularités des flots holomorphes. Ann. Inst. Fourier (Grenoble) 46 (1996), no. 2, 411-428.

[35] Rebelo, J. C.: Champs complets avec singularités non isolées sur les surfaces complexes. Bol. Soc. Mat. Mexicana (3) 5 (1999), no. 2, 359-395.

[36] Sullivan, D.: Discrete conformal groups and measurable dynamics. Bull. Amer. Math. Soc. (N.S.) 6 (1982), no. 1, 57-73.

[37] Suzuki, M.: Sur les opérations holomorphes de $\mathbb{C}$ et de $\mathbb{C}^{*}$ sur un espace de Stein. In Fonctions de plusieurs variables complexes, III (Sém. François Norguet, 19751976), 80-88. Lecture Notes in Math. 670, Springer, Berlin, 1978.

[38] Tabor, M. and Weiss, J.: Analytic structure of Lorenz system. Phys. Review A 24 (1981), no. 4, 2157-2167.

Received July 18, 2012; revised November 4, 2013.

Julio C. Rebelo: Institut de Mathématiques de Toulouse, 118 Route de Narbonne, 31062 Toulouse, France.

E-mail: rebelo@math.univ-toulouse.fr

Helena Reis: Centro de Matemática da Universidade do Porto, Faculdade de Economia, Universidade do Porto, 4200-464 Porto, Portugal.

E-mail: hreis@fep.up.pt

The second author is partially supported by the Fundação para a Ciência e Tecnologia (FCT) through CMUP, through the postdoctoral grant SFRH/BPD/34596/2007 and through the grant PTDC/MAT/103319/2008. 